Portland State University

PDXScholar

$1-1-2011$

\title{
The Relationship Between Teachers' Multicultural Attitudes and Their Instructional Practice with English Language Learners: A Mixed Method Study
}

Adria Danielle Dodici

Portland State University

Follow this and additional works at: https://pdxscholar.library.pdx.edu/open_access_etds Let us know how access to this document benefits you.

\section{Recommended Citation}

Dodici, Adria Danielle, "The Relationship Between Teachers' Multicultural Attitudes and Their Instructional Practice with English Language Learners: A Mixed Method Study" (2011). Dissertations and Theses.

Paper 141.

https://doi.org/10.15760/etd.141

This Dissertation is brought to you for free and open access. It has been accepted for inclusion in Dissertations and Theses by an authorized administrator of PDXScholar. Please contact us if we can make this document more accessible: pdxscholar@pdx.edu. 
The Relationship Between Teachers' Multicultural Attitudes and Their Instructional Practice with English Language Learners: A Mixed Method Study

by

Adria Danielle Dodici

A dissertation submitted in partial fulfillment of the requirements for the degree of

\title{
Doctor of Education
}

in

Educational Leadership: Curriculum and Instruction

\author{
Dissertation Committee: \\ Micki Caskey, Chair \\ Kenneth Peterson \\ Lynn Santelmann \\ Dannelle Stevens \\ Jacqueline Temple
}

Portland State University

(C)2011 


\begin{abstract}
Teachers of English language learners (ELLs) are often ill prepared to provide content area instruction tailored to their students' needs. This explanatory mixed method study examined the relationship between 15 ELL teachers' multicultural attitudes and use of sheltered instruction (SI). The purpose of the study was to ascertain whether teachers' multicultural attitudes influenced their use of SI. Data were gathered using a Demographic Questionnaire, Multicultural Efficacy Scale (MES), Classroom Instruction Questionnaire, and semi-structured interviews. A correlational analysis found no statistically significant relationship (i.e., $r=-.34 ; p=.22$ ) between participants' scores on SI observations and the MES. A correlational estimate for the four highest SI scorers indicated a relationship between the two sets of scores (i.e., $r=.75 ; p=.25$ ). Qualitative data did not indicate any patterns that might distinguish the high and low SIOP scorers; however, Aptitude Measurement Interaction may explain the different correlational results.
\end{abstract}

Keywords: multiculturalism, sheltered instruction, English language learners, teacher attitudes, professional development for teachers of English language learners 


\section{Dedication}

This dissertation is dedicated to my grandmother, Anna Maria Devoto Salanti, who valued education as much I do, but regrettably did not have the same opportunities. What could not have been accomplished during her lifetime has been realized during mine. I would like to express my sincere gratitude for the love and care she provided me as a child. She approached life courageously, worked extremely hard, and was generous towards others. Lessons learned from her have been instrumental in my completion of this dissertation. Grazie, nonna.

"What do we live for if it is not to make life less difficult for each other?" George Eliot

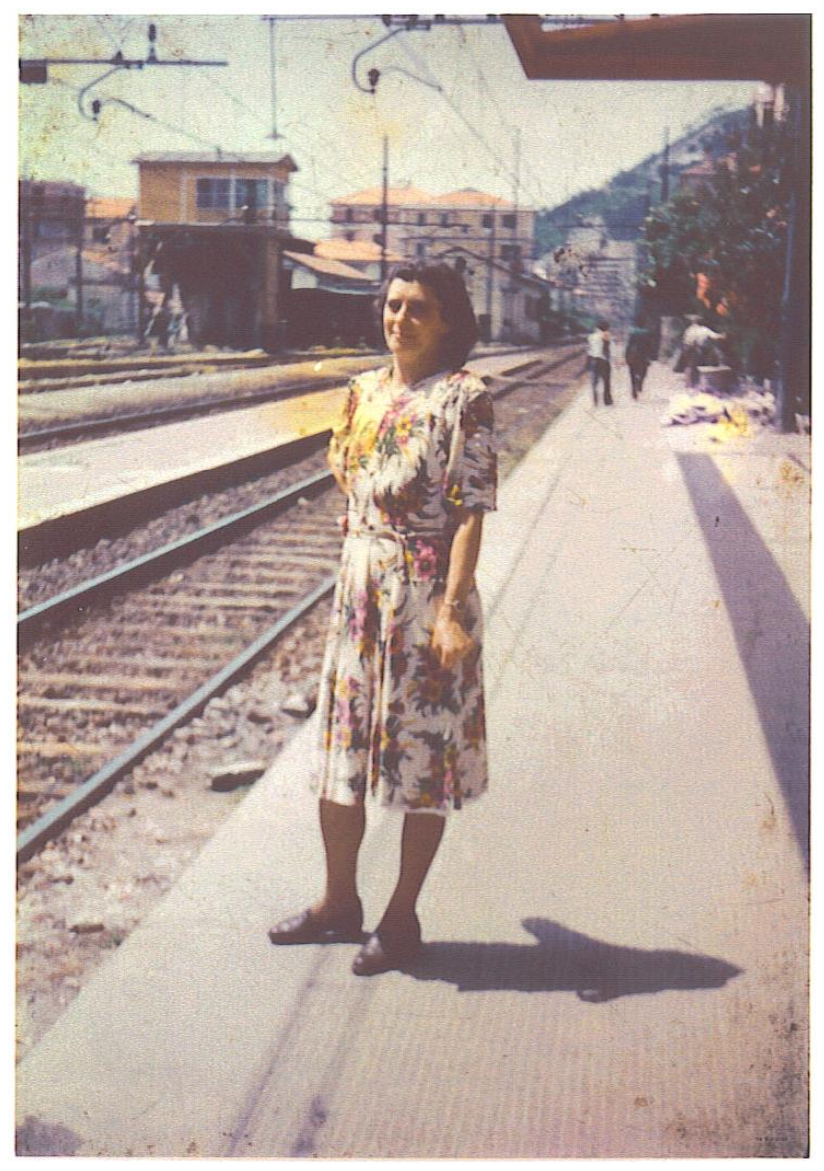

Leaving Chiavari, Genoa, Italy for the United States, 1950

1922-2004 


\section{Acknowledgements}

I would like to thank my advisor, Dr. Micki Caskey of Portland State University for her consistently thorough, timely feedback and kind, helpful manner. During this experience, I have learned a tremendous amount about scholarly writing and presenting my research to others. Dr. Caskey saw my potential and continuously encouraged me, for which I am grateful. Likewise, I am indebted to my core, specialty, and doctoral committees for holding me to high standards and not allowing substandard work to pass as acceptable when it was not and I was capable of better.

My first language arts and social studies class at Valor Middle School deserves recognition for educating me on what it means to be an effective teacher to ethnically and linguistically diverse children. My interest in helping students like them is what prompted my entrance into a doctoral program.

The teachers who bring their hearts, souls, minds, and hands to the task of educating ethnically and linguistically diverse students have my sincere respect. To truly reach and educate their students to high standards, such professionals face numerous barriers from society and the school systems in which they work. Their intelligence, dedication, compassion, and love for others are often overlooked.

Finally, I am indebted to both family and friends for the support and encouragement they have provided throughout my life. Like George Burton Adams, I realize, "There is no such thing as a 'self-made' man [sic]. We are made up of thousands of others. Everyone who has ever done a kind deed for us, or spoken one word of encouragement to us, has entered into the make-up of our character and of our thoughts, as well as our success." 
Abstract.......................................................................

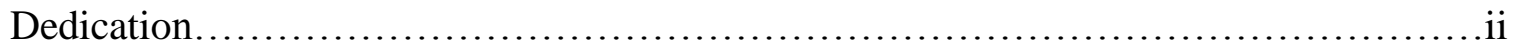

Acknowledgements..........................................................

List of Tables............................................................ vii

List of Figures...............................................................

Chapter 1

Introduction

Background of the Study ............................................... 2

Foundations of US Public Education................................. 2

Pervasive Educational Inequity in US Public Schools..................6

Ways of Addressing the Achievement Gap............................11

Attention to Diversity in Teacher Education Programs and Inservice

Professional Development......................................14

Research Study......................................................... 16

Problem Statement.................................................. 16

Purpose of the Study..............................................17

Research Questions............................................21

Research Hypothesis...........................................22

Professional Significance of the Study .............................22

Definitions...................................................23

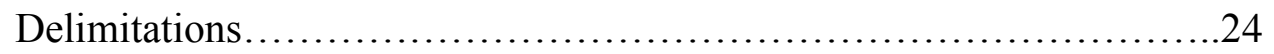




\section{Chapter 2}

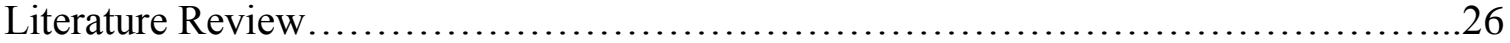

Multiculturalism.....................................................26

The Relationship Between Hegemony and Social Inequity....................28

Resistance to Cultural Hegemony..................................33

Effective Instructional Approaches to Teaching English Language Learners......36

Sheltered Instruction and Related Theories of Second

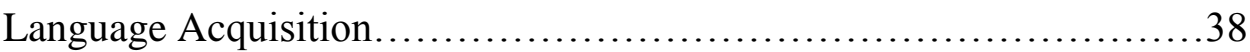

The Role of Input........................................41

The Role of Output......................................44

The Role of Negotiating Meaning...........................45

Rigorous Content Standards Coupled with High Expectations...........48

Sheltered Instruction and Multicultural Education...........................49

Relationship Between Teacher Beliefs and Instructional

Practice.......................................................5

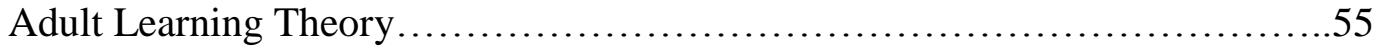

Core Adult Learning Principles...................................55

Effective Practices in the Professional Development of Teachers.......6 61

Conceptual Framework...............................................6. 63

Relationship Between the Literature Review and Proposed Study...............65

Chapter 3

Methodology ...............................................................68

Research Design.....................................................69

Context and Access.................................................... 70

Participants and Selection Process......................................... 70

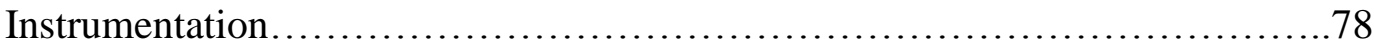

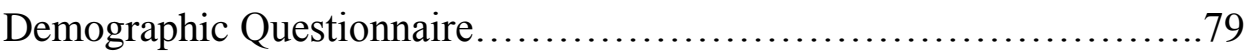

Multicultural Efficacy Scale.....................................80

Sheltered Instruction Observation Protocol............................83

Classroom Instruction Questionnaire..............................8 85

Semi-structured Interviews.......................................... 86

Issues of Validity Specific to Qualitative Data.........................88

Data Collection.........................................................91

Measuring Variables............................................92

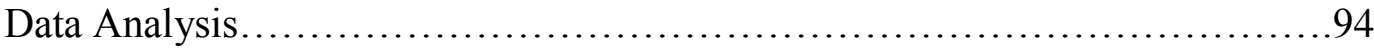

Quantitative..................................................... 98

Qualitative..................................................... 100

Representing and Reporting Findings.................................. 102

Quantitative...................................................103

Qualitative..................................................103 
Chapter 4

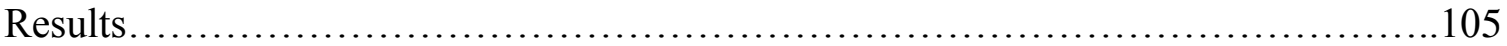

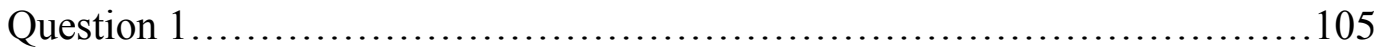

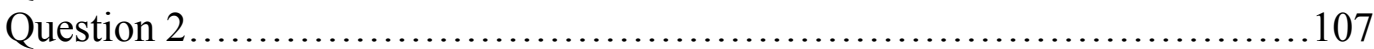

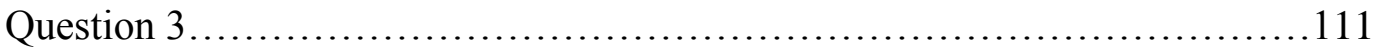

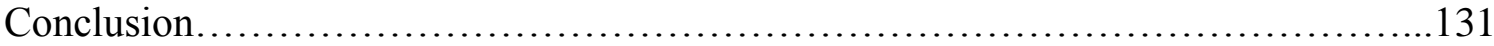

Chapter 5

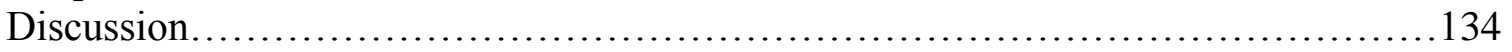

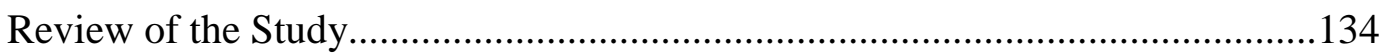

Statement of the Problem..............................................134

Purpose of the Study ................................................134

Research Hypothesis and Questions.................................135

Review of Methodology .........................................135

Results of the Study.............................................

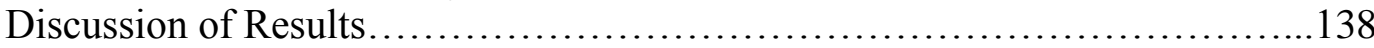

Recommendations for Further Study ..............................142

Implications for Practice............................................143

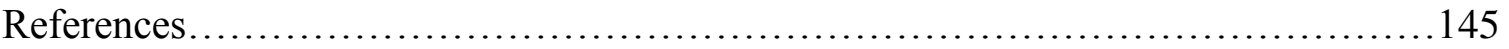

Appendices

Appendix A: Demographic Questionnaire..................................157

Appendix B: Multicultural Efficacy Scale .....................................159

Appendix C: Permission to Use the Multicultural Efficacy Scale.................164

Appendix D: Sheltered Instruction Observation Protocol........................166

Appendix E: Permission to Use the Sheltered Instruction

Observation Protocol.............................................168

Appendix F: Classroom Instruction Questionnaire..........................170

Appendix G: Semi-structured Interview Questions...........................175

Appendix H: Codebook of Quantitative Data..................................176

Appendix I: Start List of Codes for Analyzing the Classroom Instruction Questionnaire....................................180

Appendix J: Distributions of the Multicultural Efficacy Scale-Skewness and Kurtosis. 


\section{List of Tables}

Table $1 \quad$ NAEP Mathematics and Reading Test Scores-

Between Group Difference................................... 8

Table 2 Personal Background of Participants................................72

Table 3 Participants' Education and Training Related to Teaching

English Language Learners.

Table 4 Descriptive Statistics for Multicultural Efficacy

Scale Scores-Central Tendency..........................105

Table 5 Descriptive Statistics for the Multicultural Efficacy

Scale Scores-Variability

Table 6 Descriptive Statistics for the Multicultural Efficacy

Scale Scores-Rank Based on Total Subscale B-D Scores

Table 7 Descriptive Statistics for the Sheltered Instruction

Observation Protocol Observations 1 and 2-

Central Tendency.

Table 8 Descriptive Statistics for the Sheltered Instruction

Observation Protocol Observation 1-Variability

Table 9 Descriptive Statistics for the Sheltered Instruction

Observation Protocol Observation 2-Variability

Table 10 Descriptive Statistics for Sheltered Instruction

Observation Protocol-Rank Based on the

Sum of the Two Observations.

Table 11 Two-tailed Pearson Product-moment Correlation Test of

the Total SIOP and MES Scores on Subscales B through D......112

Table 12 Participants' Description of How Often They Use

Sheltered Instruction Techniques from

the Quantitative Section of the Classroom

Instruction Questionnaire

Table 13 Participants' Description of How Often They Use Sheltered

Instructional Techniques and Their Total SIOP

Observation Score Reported as Percentages....

Table 14 Descriptive Statistics of the Quantitative Section

of the Classroom Instruction Questionnaire-

Central Tendency.

Table 15 Descriptive Statistics for the Quantitative Section

of the Classroom Instruction Questionnaire-Variability. ...

Table 16 Descriptive Statistics for the Quantitative Section

of the Classroom Instruction Questionnaire-

Rank Based on Total Score.

Table 17 Descriptive Statistics for all Quantitative Measures-

Rank Based on Total Scores.

Table 18 Case-ordered Descriptive Matrix for Interview Data... 


\section{List of Figures}

Figure 1 Prism Model of Second Language Acquisition.......................................38

Figure 2 Path to Educating English Language Learners

Successfully..........................................63

Figure 3 Highest Educational Degree Obtained by Participants' Fathers...........73

Figure 4 Highest Educational Degree Obtained by Participants' Mothers..........73

Figure 5 Highest Educational Degree Obtained by Participants....................74

Figure 6 Participants' Socioeconomic Status as Children.........................74

Figure $7 \quad$ Participants' Socioeconomic Status as Adults.......................... 75

Figure 8 Total Hours of Participants' Sheltered Instruction Training...............76

Figure 9 Total Years of Participants' Middle School Teaching Experience.........77

Figure 10 Total Years of Participants' Experience Teaching at Least One

English Language Learner....................................77

Figure 11 Flowchart of Quantitative and Qualitative Data Analysis...............95

Figure 12 Participants' Description of the Challenges to Teaching Content with Multicultural Educational Practices.....................122

Figure 13 Participants' Description of the Benefits to Working with Diverse Students..............................................123

Figure 14 Participants' Description of the Challenges to Working with Diverse Students.......................................124 


\section{Chapter 1}

\section{Introduction}

Throughout U.S. history, people have advocated for a public educational system that offers all students an equal opportunity to obtain a quality education, an education that leads to a fulfilling life and includes active participation in a democratic society. Some contend that such an educational system exists. Others describe the existence of an equitable U.S. public educational system as an illusion (Darder, 1991: McLaren, 2003). Ongoing discourse about the importance of education is critical. "When citizens deliberate about the education of the young, they are also debating the shape of the future for the whole nation" (Mondale \& Patton, 2001, p. 2). In this paper, I assert that an equitable educational system has not been achieved for all students in the U.S., specifically for English language learners. Moreover, I examine the causes of inequity in the educational system and conduct a research study of two contributing factors in the maintenance of an educational system that do afford each English language learner a quality education. The two contributing factors under examination are (a) establishing a learning environment in which students' cultural and linguistic diversity is honored and respected and (b) providing English language learners content area instruction that is academically challenging, tailored to their linguistic proficiency, educational background, and academic needs (Teachers of English to Speakers of Other Languages [TESOL] 1995;1999). Finally, based on the results of my research study, as well as recommendations from the literature on professional development for teachers. I suggest implications for providing effective professional development for teachers of English language learners. 


\section{Background of the Study}

Foundations of U.S. public education. The Greek philosophers Socrates, Aristotle, and Plato laid the foundation for current views about how children in the U.S. should be educated. Socrates described knowledge as virtuous, eternal, and universal. He believed the purpose of education was to develop the inherent knowledge or virtue in the individual and advance his ability to reason (Plato, 1900). Such an education required learners to re-examine and reconstruct their ideas via questioning (Mayer, 1966).

Because Socrates believed a society was as good as the teachings it provided its citizens, he asserted it was ethically and morally important for all men to be knowledgeable (Plato, 1900).

While Socrates focused on the role of education in the individual's development, Plato was concerned with the cohesion of the state and the contribution of each individual to the maintenance of that unity. According to Plato (n.d.a), the purpose of law was not to make each citizen happy; rather, each citizen was to share the skills of his or her class with the community, thereby unifying the city. Although Plato did not support providing the same education to all, he did maintain all the state's inhabitants, including females, should have some training due to the state's need for citizens with various skills. Moreover, Plato (n.d.b) asserted education should be compulsory. Plato's description of school curriculum included reading, mathematics, logic, physical activity, and music.

Aristotle concurred with Plato that education was important to the function of society and should be supported by the state. Aristotle argued the stability of a society depended upon the intelligence and morality of its citizens. Therefore, it was in the best 
interest of the society to maintain itself via the education of its citizens (Aristotle, trans.1997; Power, 1991).

Aristotle (1997) perceived a connection between education, character formation, and social ideals. Education that supported the development of an individual's character was to be supplemented by preparatory training for various crafts and professions. Like Plato, Aristotle did not think all inhabitants of the state should receive the same education. Aristotle also did not believe the state should provide girls an education. For Aristotle, the education of free persons and slaves was to be different. The goal of a free person's education was to prepare his mind and body for exercising virtue. His education included a core curriculum of reading, writing, music, gymnastics, and in some cases drawing. In contrast, the purpose of a slave's education was to prepare the individual for required work.

Over two millennia later and after having won independence from Britain, Americans focused on building a nation from the original 13 colonies. Like the aforementioned Greek philosophers, many Americans believed schools could play a critical role in educating the population for the survival of democracy (Mondale \& Patton, 2001). In a 1786 letter to George Wythe, Thomas Jefferson (1904) argued that because kings, nobles, and priests were not good conservators of public happiness, it was necessary to,

Preach ... a crusade against ignorance; establish and improve the law for educating the common people. Let our countrymen know, that the people alone can protect us against these evils, and that the tax which will be paid for this purpose, is not more than the thousandth part of what will be paid to kings, 
priests, and nobles, who will rise up among us if we leave the people in ignorance. (p. 397)

In his first address to Congress regarding the importance of education, George Washington stated, "There is nothing which can better deserve your patronage than the promotion of science and literature. Knowledge is in every country the surest basis of public happiness..." (1790, para. 11). Public education in the U.S. was created in the middle nineteenth century with common schools. Students were not charged tuition; instead, local property taxes were collected to fund the schools. Local school committees primarily governed each school with a small amount of state regulation. The common schools were open to all White children, with the education of Blacks being quite limited and often taking place in segregated schools (Mondale \& Patton, 2001; Webb, Metha, \& Forbis, 2003).

Populations defined the purpose of the common schools differently. Many working-class individuals believed the common schools provided the opportunity for achieving upward mobility, the education required to protect themselves from the oppressive conditions created by the upper class, and the requisite education for participating in a democracy. Business and industry leaders, who supported common schools, viewed them as suppliers of literate, trained workers (Webb et al., 2003). In response to the increase in immigration from Ireland, Germany, and Southern Europe, the majority of the dominant, upper class, English-speaking Protestants supported using the common schools as an instrument of assimilation (Gutek, 1991). Unlike previous northern European immigrants to the U.S., subsequent immigrants did not share the language or many of the beliefs and values of the dominant class. The dominant class 
responded by using public education to eliminate such differences. Lastly, many individuals believed the pursuit of knowledge elevated human existence and deterred people from immoral conduct (Binder, 1974). Therefore, common schools were perceived as a means to control crime and social unrest.

Jefferson and Aristotle shared the belief that education should be supported by the state and was essential to a functioning society. However, groups of children in both civilizations were excluded from the public happiness to which Washington referred, the crusade against ignorance described by Jefferson, and the Athenian preparation for the exercise of virtue.

A century after the creation of the common schools, educational theorist, John Dewey (1916), advocated for using education to form the citizen as a contributor to society, not to simply form the individual. He conceptualized education as a force capable of releasing an individual's capacity toward addressing social or collective goals. Dewey also expressed concern about the potential inherent in an educational system to exacerbate inequity in society. He described the division of society into classes in which some classes serve as tools for the higher culture of others. According to Dewey, not only must education not be used to facilitate the exploitation of one class for another's benefit, but school facilities and equipment must be of equal quality and availability for all students. Family resources must be supplemented so students may take advantage of schooling. Furthermore, Dewey argued that a modification of traditional ideas of culture, subjects of study, and methods of teaching and discipline was required to keep all youth in the educational system until they acquired the knowledge and skills necessary to manage their own economic and social careers. 
To the detriment of female, minority, and poor students in U.S. public schools, Dewey's advice, by in large, has not been heeded. Instead, federal, state, district, and school policies have been established that deny particular student populations equitable access to learning. Such policies include, but are not limited to (a) the absence of federal provisions for livable wages, stable housing, adequate health care, and quality early-child education for minority and low-income children (Rothstein, 2004); (b) inequitable school funding at state and district levels (McClure, Wiener, Roza, \& Hill, 2008); (c) the passage of state English-only laws, such as California's Proposition 187, which encourage districts and schools to use inappropriate educational programs for English language learners (i.e., the absence of bilingual education and limited specialized English instruction for English language learners) (Broder, 1998); and (d) the lack of culturally responsive instruction in schools (Zeichner, 2003).

Pervasive educational inequity in U.S. public schools. High school dropout rates, quality of instruction indicators, national content area assessment results, and school funding allocations are illustrations of the inequity that exists between various ethnic, racial, and socioeconomic populations of U.S. schoolchildren. High school graduation rates for 2007 clearly indicate all students are not educated to the same educational standards. At $21.4 \%$, Hispanics represent the majority of the 16 to 24 -year olds who have not obtained a high school diploma or equivalent degree. Blacks comprise $8.4 \%$ of students who have not graduated from high school. Whites, who have the lowest high school dropout rate, do not complete high school at a rate of 5.3\%. Individuals at the lowest income quartile do not finish high school at a rate of over five times the rate of individuals at the highest income quartile (Snyder, Dillow, \& Hoffman, 2009). 
Test scores provide another example of the discrepancy between student populations in U.S. public schools. The National Assessment of Educational Progress (NAEP), administered in grades 4,8 , and 12 , is an assessment used by the U.S. Department of Education to measure students' progress in various subject areas. Students who score at the proficient level are believed to have demonstrated competency over challenging subject matter (KewalRamani, Gilbertson, Fox, \& Provasnik, 2007). Results from the 2005 NAEP demonstrated a significant achievement gap between two groups of students: (a) Blacks, Hispanics, and American Indian/Alaska Natives and (b) Whites and Asian/Pacific Islanders. On the measure of reading achievement at the twelfth grade level, achievement gaps existed between the percentage of Whites and all other reported groups. Blacks scored 27\% below Whites, Hispanics 23\% below Whites, Asian/Pacific Islanders 7\% below Whites, and American Indian/Alaskan Natives 26\% below Whites. Based on twelfth grade mathematics assessment results, all groups except Asian/Pacific Islanders scored below Whites. Blacks scored 23\% below Whites, Hispanics 21\% below Whites, and American Indian/Alaskan Natives scored 23\% below Whites. Conversely, Asian/Pacific Islanders outperformed Whites by 7\% (KewalRamani et al., 2007).

A review of NAEP results in mathematics and reading prior to 2007 indicate a pattern of Whites consistently outperforming both Blacks and Hispanics (Perie \& Moran, 2005). The test performance gap among 17 -year-olds over a period of 29 years is noteworthy (see Table 1). Percentages refer to the number of students scoring between 250 and 299 on reading and 300 to 349 on mathematics. Students who scored at these levels are believed to have demonstrated the ability to connect ideas and make 
generalizations in reading and perform moderately complex procedures and use logical reasoning to solve problems in mathematics.

Table 1

NAEP Mathematics and Reading Test Scores-Between-Group Difference

\begin{tabular}{lcccc}
\hline Year & \multicolumn{2}{c}{ Reading } & \multicolumn{2}{c}{ Mathematics } \\
\hline & Whites-Blacks & Whites-Hispanics & Whites-Blacks & Whites-Hispanics \\
\hline 1975 & $43 \%$ & $33 \%$ & N/A & N/A \\
1978 & N/A & N/A & $41 \%$ & $34 \%$ \\
1980 & $43 \%$ & $25 \%$ & N/A & N/A \\
1982 & N/A & N/A & $38 \%$ & $33 \%$ \\
1990 & $19 \%$ & $13 \%$ & $30 \%$ & $33 \%$ \\
1999 & $21 \%$ & $19 \%$ & $43 \%$ & $32 \%$ \\
2004 & $19 \%$ & $16 \%$ & $37 \%$ & $32 \%$ \\
\end{tabular}

Note. Percentages refer to the number of students scoring between 250 and 299 on reading and 300 to 349 on mathematics.

Significant discrepancies also exist in terms of public school funding. National 2004-2005 data on states' average per pupil spending indicated a $\$ 8,901$ difference between New Jersey, the state that spent the most per student (i.e., \$14,117) and Utah, the state that spent the least per student at (i.e., \$5,216) (Hill, Roza, \& Harvey, 2008). Public school funding inequity exists across states, districts, and schools within a particular district. Financial support is provided to schools with locally raised property/levy monies, state tax revenues, and federal dollars intended for specific students and programs (Hill et al., 2008). Of the total funding provided to schools, the federal government supplies approximately $9 \%$, local education agencies provide $44 \%$, and states supply $47 \%$ of the funding (Snyder et al., 2009). The purpose of federal funding such as Title I is to ameliorate the effects of poverty on educational achievement. Because $91 \%$

${ }^{1}$ Data not available 
of a school's money comes from locally raised property/levy monies and state tax revenue, it is essential for this non-federal funding to be equalized among low and high poverty schools before Title I funds are distributed. This allows federal money to provide the extra resources for which it was intended (McClure et al., 2008).

While there are several comparability provisions written into the law with the intention of balancing funding between schools before applying federal funds, the existence of the provisions does not ensure fiscal equity for Title I schools (McClure et al., 2008). Primary problems with comparability provisions include (a) allowing districts to ignore differences in teacher salaries across schools; (b) not requiring full fiscal equity between schools, with only $90 \%$ being required; (c) limiting the amount of additional state and local funds that can be provided to high-poverty, Title I schools to no more than $10 \%$ in excess of the amount provided to non-Title I schools; and (d) lenient enforcement of the comparability provisions.

The problem of educational inequity is compounded when staffing of higherincome and lower-income schools is examined. Higher-income schools tend to attract the highest paid teachers. Additionally, the majority of districts permit senior teachers to choose their work site, which leads to significantly varied salary expenditures between similarly sized schools (Hill et al., 2008). High-poverty schools have substantially more less experienced, lower paid teachers and higher turnover rates than low-poverty schools (McClure et al., 2008; Orfield \& Lee, 2005). Also, compared to their peers in lowminority, low-poverty schools, high school students in high-minority, high-poverty public schools were more likely to be taught English, science, and mathematics by out-of-field teachers (i.e., teachers who did not major or become certified in the subjects they teach) 
(Wirt et al., 2004). The extent of the problem is further illustrated by considering the significance of the relationship between teacher quality and student academic performance.

In a study on teacher and classroom context effects on student achievement, researchers used the Tennessee Value-Added Assessment System (TVAAS) database to measure the size of teacher effects on student learning while simultaneously considering the influences of intraclassroom heterogeneity, student achievement level, and class size on academic growth (Wright, Horn, \& Sanders, 1997). The TVAAS, a database of longitudinal student, teacher, school, and school system information was designed to estimate system, school, and teacher effects on the academic gains of students. Using third, fourth, and fifth grade data from five subsets of the Tennessee Comprehensive Assessment Program (TCAP) test, students' between-year academic progress was determined and 30 separate analyses were conducted. The researchers found the teacher effect to be highly significant in every analysis with a larger effect size than any other factor in 20 of 30 analyses. Effective teachers appeared to be successful with students of all achievement levels and regardless of the amount of heterogeneity of their classes. The achievement level of the student had the largest effect size in 10 of 30 analyses, with the overall school system being the third most important factor affecting student achievement. In conclusion, teacher quality was identified as the most important factor affecting student learning.

In a study of the cumulative effects of teachers on student achievement, Sanders and Rivers (1996) found students with comparable abilities and initial achievement levels may have significantly different academic outcomes due to the sequence of teachers to 
which they were assigned. On fifth grade mathematic achievement tests, students placed with low performing teachers for three consecutive years scored on average at the $44^{\text {th }}$ percentile, while those placed with high performing teachers for three consecutive years scored on average at the $96^{\text {th }}$ percentile, demonstrating a 52-point range between the average scores of the two student groups. Further highlighting the importance of quality teachers, Sanders and Rivers (1996) determined the residual effect of both very effective and ineffective teachers was measurable up to two years after they had taught students. A more recent teacher impact study on average student math performance of third to fifth grade students in Los Angeles Unified School District found an average difference of 10 percentile points on mathematics tests between students assigned to top and bottomquartile teachers (Gordon, Kane, \& Staiger, 2006).

Ways of addressing the achievement gap. Recognizing the powerful effect quality teaching has on learning, Gordon and his colleagues (2006) asserted matching the most effective teachers with the neediest students in schools could close gaps in educational achievement. Zeichner (2003) supported this notion by identifying three key instructional practices required for all students to achieve high standards: (a) high expectations of all students, (b) cultural responsiveness in instruction, and (c) the use of teaching strategies for making meaning.

Though an essential component of student success, having high expectations of all students is not the norm for all educators. Even in the face of contradictory evidence, many students in teacher education programs persist in believing some students cannot learn and therefore hold lower expectations of them (Goodlad, 1990; Pang \& Sablan, 1998). Supporting this point, research has indicated many teacher education students 
have negative attitudes about racial, ethnic, and linguistic groups that differ from their own (Valli, 1996). Teachers' judgments are powerfully influenced by preconceptions of students' individual traits and the characteristics of students' families and communities (Elmore \& Fuhrman, 2001). A study of sixth grade teachers indicated the effects of the teachers' expectations were significantly stronger among African Americans, students from lower socioeconomic status, students with low self-concepts, and those with a prior history of low academic achievement (Jussim, Eccles, \& Madon, 1996). Kunjufu (2002) asserted the most important factor affecting the academic achievement of AfricanAmerican children is the teachers' expectations of them.

In his observations of a class of kindergarten students in both their kindergarten and second grade years, Rist (2000) found that the public school the students attended reflected and reinforced the class system in the larger society. Teachers grouped students into table groups they believed to be smart, of average intelligence, and slow. Rist further observed teachers also used criteria such as family income, education, and family dynamics to group students (i.e., the number of children in the family and whether both parents were present in the home). Those from families who shared characteristics most in common with middle class families were given the highest status in the classroom by their teachers. The manner in which students were grouped in kindergarten continued into first and second grade with the lower-status kindergarten students experiencing no upward mobility. Lower status students disproportionately experienced a lack of communication with the teacher, lack of involvement in class activities, less instructional time from the teacher, denial of privileges given to others, greater physical distance between their table groups and the teacher, higher incidence of control-orientated 
behavior directed toward them, and poorer quality of instruction (e.g., the teacher consistently wrote on a section of the blackboard only the high-status students could see). Rist asserted that the issues of color and class inequality in U.S. society are at the core of the future of U.S. education. A profound chasm exists between the rhetoric of opportunity and the reality of American society for those who are the most impoverished in the U.S.

As the public school student population becomes increasingly culturally, linguistically, and ethnically diverse, as well as poor (Garofano \& Sable, 2008; Lindholm-Leary, 2000; Tang, Sable, \& Hoffman, 2009), the teaching population continues to be overwhelmingly White, female, and middle class (National Education Association, 2003; Strizek, Pittsonberger, Riordan, Lyter, \& Orlofsky, 2006). Many preservice White teachers entered the profession with little cross-cultural background, knowledge, and experience and had a limited understanding of discrimination, particularly racism (Su, 1997). After completing their coursework, the majority of White, preservice teachers felt ill prepared to teach in multicultural classrooms or communicate with diverse children and their families (Barry \& Lechner, 1995). Contrasting their White counterparts, preservice teachers of color were found to demonstrate a higher commitment to multicultural teaching, social justice, and providing students of color with an academically challenging curriculum (Rios \& Montecinos, 1999; Su, 1997). Rios and Montecinos clarified that an individual's ethnicity alone does not determine his or her success with teaching students of color. Rather, the manner in which individuals are socialized into the teaching profession and how they come to understand diversity are critically important in their preparation to work with ethnic minority students. Valli 
(1996) asserted a personal bond must be created between teachers and students, if teachers are to address students' psychological and social development, in addition to their academic development.

Culturally responsive teaching provides the means to bridge home and school cultures, so all students may be taught to high standards (Zeichner, 2003). In culturally responsive teaching, students' languages, cultures, and life experiences are incorporated into the academic and social context of schooling. Additionally, the school's codes and customs are explicitly taught to students to support students' full participation in the classroom and school as a whole. Effective teachers use their understanding of their students' cultures to adapt curriculum and instruction as necessary.

Although students of color and English language learners tend to receive instruction that focuses on decontextualized skills (Moll, 1988), instruction that centers on making meaning out of content seems to be the most successful with this group of students (Zeichner, 2003). An example of an approach that focuses on meaning making is the reciprocal interaction model (Cummins, 1986). In this model, students are not considered empty vessels that need to be filled with the teacher's knowledge. Rather, the role of the teacher is to guide and facilitate instruction through the use of dialogue. Higher-level cognitive skills, collaborative learning, and reading and writing as a means to learning are emphasized.

\section{Attention to diversity in teacher education programs and inservice}

professional development. In a review of teacher education programs, Gollnick (1995) found most teacher education programs had a limited number of substantive programs or courses that addressed issues of diversity. Very few teacher education programs 
integrated issues of diversity throughout the curriculum. Instead, a segregated approach is more commonly used in which cultural diversity is the focus of a single course or is a topic in a few courses and not part of the other components in the program (Zeichner \& Hoeft, 1996). Many student teachers from such programs significantly struggled when placed in classrooms consisting of students who were culturally or linguistically different from themselves (Birrell, 1994; Goodwin, 1994; Weiner, 1990). Such programs lacked coherence between courses and were unsuccessful in teaching students to design instruction to address classroom and community contexts (Barone, Berliner, Blanchard, Casanova, \& McGowan, 1996).

Effective professional development programs assist teachers in understanding the complexity of ethnic groups and the ways race, ethnicity, language, and social class influence student behavior. To address the increasing cultural and ethnic gap between teachers and students, Banks and colleagues (2005) recommended that professional development programs assist teachers in (a) revealing and identifying their personal attitudes toward racial, ethnic, linguistic, and cultural groups; (b) acquiring knowledge about the histories and cultures of the diverse groups in the U.S.; (c) becoming aware of the varied perspectives that exist within different ethnic and cultural communities; (d) understanding the ways in which institutionalized knowledge within schools, universities, and popular culture can perpetuate stereotypes about racial and ethnic groups; and (e) obtaining the knowledge and skills necessary to develop and implement equity pedagogy, or instruction that provides all students an equal opportunity to attain academic and social success in school. 
Because many teacher education programs do not successfully prepare teachers to educate students of color, the importance of inservice professional development that addresses this need becomes readily apparent. Zeichner and Hoeft (1996) conducted a review of the literature on staff development and found discussions of the various purposes of staff development, particularly specific teaching skills and effective practices in planning and delivering staff development. However, the researchers concluded that recommendations in the staff development literature were insufficient in guiding the complex and demanding personal and professional changes required for teachers to become more adept at culturally relevant instruction. If students of color are to receive the education they deserve, teachers must have access to training for culturally relevant instruction as preservice teachers and throughout their careers as educators.

\section{Research Study}

Problem statement. Nieto (2005) aptly described both the potential and failure of the U.S. public school system:

...public education in the United States has been characterized by both extraordinary achievement and abysmal disappointments, and by everything in between. At the same time, it has remained the best hope for personal fulfillment and a more productive life for most segments of our population. (p. 52)

Because of the influence that education has on people's lives, closing the achievement gap is an ethical imperative. Numerous studies have identified essential characteristics of schools if educational equity is to be afforded all students. Such studies advocate providing students access to challenging curricula and instruction; high-quality 
teachers who have the content knowledge, pedagogy, and high expectations required to teach challenging content to a diverse group of learners; and necessary additional supports to ensure student success (Manning \& Kovach, 2003). The conditions necessary for closing the achievement gap have been identified, yet the teacher education and inservice professional development of most teachers does not adequately prepare them to address the needs of their ethnically, culturally, linguistically, and socioeconomically diverse students (Zeichner \& Hoeft, 1996). The problem is teachers of English language learners are often inadequately prepared to provide content area instruction.

Purpose of the study. The primary purpose of this study is to examine the relationship between teachers' multicultural attitudes and their use of sheltered instructional techniques in the classroom. This relationship is examined with the intention of informing practice in the field of professional development for teachers of English language learners. Students who are learning English rely on qualified teachers who can instruct them in a manner that develops their English proficiency and academic knowledge. However, the professional development of teachers of English language learners has not been given its due attention. Rather, the professional development of English language learner teachers is one of the most unexamined and overlooked areas of preservice and inservice teacher education in the U.S. (Minaya-Rowe, 2002). Therefore, a secondary goal of conducting this research is to improve the professional development of the English language learner teachers at the research site. The ultimate goal is to provide more English language learners the quality education they deserve.

For the purposes of my research study, I focus on English language learners and the professional development of their teachers for several reasons. First, the numbers of 
English language learners in U.S. public schools continues to rise substantially. Between the 1994-1995 and 2004-2005 school years, the number of English language learners in U.S. schools increased 60.76\% (National Clearinghouse for English Language Acquisition [NCELA], 2006). As the population of English language learners continues to increase, the need for educators to understand fully how to educate this population of students will become even more of a priority. Second, English language learners are one of the most vulnerable, underserved students in U.S. public schools (Nieto, 2005). Latinos, the largest and fastest growing population of English language learners (Lindholm-Leary, 2000), have one of the highest rates of poverty (U.S. Census Bureau, 2006), and the highest high school drop out rate (Snyder et al., 2009). Third, several factors that contribute to the underachievement of English language learners need to be addressed including (a) inappropriate educational programs based on the inaccurate assumption that what is effective for native-English speakers will be as effective for English language learners (LaCelle-Peterson \& Rivera, 1994), (b) a shortage of qualified teachers of English language learners (Lucas \& Grinberg, 2008; National Public Radio, 2002), (c) teachers' low expectations of English language learners and inaccurate perception of them as low-performing native-English speakers (Waxman \& Padrón, 2002), and (d) the use of ineffective teaching practices prevalent in English language learner classrooms (Echevarría, Vogt, \& Short, 2008). Ineffective teaching practices for English language learners include, but are not limited to, (a) denying or failing to acknowledge students' culturally specific life experiences and the role they play in supporting the learning of new concepts, (b) designing activities that do not require students to communicate with each other to practice English or engage in hands-on 
problem solving in which higher level thinking is necessary, (c) assigning students activities that are not age-appropriate, cognitively complex, or connected to their lives, (d) utilizing ineffective language teaching practices such as memorizing isolated vocabulary words and teaching grammar out of context (Collier \& Thomas, 2009). The internationally recognized professional organization, Teachers of English to Speakers of Other Languages' (TESOL) clearly articulated the specific needs of English language learners acquiring English and learning academic content. TESOL (1995; 1999) recommended that English language learners (a) be provided opportunities to further develop and/or use their first language for academic and social development, (b) learn in an environment in which their cultural and linguistic diversity is honored and respected, (c) be comprehensively taught English as a second language to prepare them for content material in English, and (d) receive content area instruction that is academically challenging, tailored to their linguistic proficiency, educational background, and academic needs.

This study examines the relationship between two of TESOL's recommendations: respecting and honoring cultural and linguistic diversity and providing English language learner students challenging content area instruction tailored to their needs. Sheltered instruction or Specially Designed Academic Instruction in English (SDAIE) is an approach used for teaching content to English language learners so that subject matter concepts are comprehensible and the students' English language development is supported (Echevarría et al., 2008). By using this instructional approach, teachers maintain the rigor of the subject while using specific strategies to make the instruction understandable to English language learners. When content area instruction is not 
delivered in a manner that allows English language learners to understand it, the academic success of such students is seriously compromised.

Acknowledging that the majority of teachers are not prepared to meet the needs of their diverse students (Echevarría et al., 2008; Zeichner \& Hoeft, 1996), administrators at the research site have provided substantial, long-term professional development in sheltered instruction to teachers. Over a five-year period, approximately 25 teachers received sheltered instruction professional development that was facilitated by an individual from the Center for Language Minority Education and Research. Aligned with recommendations for high-quality professional learning, the sheltered instruction professional development the teachers received was (a) embedded in their work and took place during the school day; (b) included opportunities for practice, research, and reflection; (c) focused on deepening teachers' content knowledge and pedagogical skills; and (d) was founded on a sense of collegiality and collaboration among teachers and between teachers and principals to solve significant problems related to teaching and learning (Sparks, 2002). Five different groups of teachers were instructed by the facilitator over a period of three or five days. During each session, the facilitator led the group in (a) describing the receptive and productive skills of English language learners in the beginning to advanced stages of English acquisition, (b) understanding the components of a sheltered instruction lesson, (c) reading about and discussing the benefits of differentiated instruction and cooperative learning, (d) becoming familiar with various strategies and resources supportive of instruction for each of the components in an sheltered instruction lesson, and (e) using the lesson study cycle to collaboratively choose a lesson and set goals, plan the lesson, teach or observe the lesson, discuss and 
reflect on the lesson to suggest improvements and subsequent instructional steps, and adapt the lesson based on the discussion and reflection (Richardson, 2004). Once all five groups of teachers had been trained in sheltered instructional techniques, the facilitator returned to the site to work with administrators and teacher-leaders to develop a means of continuing teachers' development as sheltered instruction teachers in her absence.

Though the sheltered instruction facilitator designed professional development sessions that incorporated effective practices and returned to the site to support continued learning in her absence, the investment in sheltered instruction professional development yielded inconsistent results with some teachers implementing sheltered instructional techniques to a satisfactory level, while others did not. In some cases, those who had the least amount of training and teaching experience were the most adept at using the techniques. This phenomenon may be related to teachers' attitudes and beliefs about instructing diverse students. I tested this hypothesis by collecting and analyzing data in response to the following research questions.

\section{Research questions.}

1. What are teachers' levels of multicultural efficacy as measured by the Multicultural Efficacy Scale?

2. What are teachers' levels of implementation of sheltered instructional techniques in the classroom as measured by the Sheltered Instruction Observation Protocol?

3. What is the relationship between teachers' attitudes about multiculturalism as measured by the Multicultural Efficacy Scale and their level of 
implementation of sheltered instructional techniques in the classroom as measured by the Sheltered Instruction Observation Protocol?

Research hypothesis. Teachers who have positive attitudes toward multiculturalism including multicultural education are more likely to implement sheltered instructional techniques. Multiculturalism is defined as the state or condition of emphasizing pluralism, confronting racism and biases, and seeing reality from a variety of perspectives (Nieto \& Bode, 2008). The components of multicultural education include (a) content integration, (b) the knowledge construction process, (c) prejudice reduction, (d) equity pedagogy, and (e) empowering school culture and social structure (Banks, 2004). My contention is that teachers who have positive attitudes toward multiculturalism are more likely to acknowledge the needs of English language learners are not the same as students who are native speakers of English, and therefore provide English language learners with instruction specific to their needs (i.e., sheltered instruction).

Professional significance of the study. This study informs practice in the field of professional development for teachers of English language learners. The current trend is to focus on training teachers in specific, instructional techniques found to be effective with English language learners while largely ignoring topics of multicultural education, such as social justice. It may be more effective for professional development to focus initially on teachers' beliefs and attitudes related to multicultural education and secondarily on other instructional methods necessary for developing English language learner students' linguistic proficiency and academic content knowledge. 


\section{Definitions.}

1. Attitude - a predisposition that consistently affects actions (Richardson, 1996);

2. African American - a black American of African descent;

3. Belief - psychologically held understanding, premise, or proposition about the world that are felt to be true (Richardson, 1996);

4. Black - comprehensive term that includes people of African descent from around the world (Nieto \& Bode, 2008);

5. Cultural diversity — differences related to race, ethnicity, social class, and language (Zeichner \& Hoeft, 1996);

6. Dominant culture - social practices that affirm the central values, interests, and concerns of the social class in control of material and symbolic wealth in society (McLaren, 2003);

7. English language learner — an individual whose first language was not English and who is not yet proficient in English;

8. Hispanic - a term used in the U.S. to refer to people living in the U.S. whose ancestors originate predominantly from one or more Spanish-speaking countries (Oboler, 1995);

9. Latino - an immigrant or U.S. born individual whose origins or those of his or her ancestors originated in Latin America;

10. Minority — refers to American Indians, African Americans, Latinos or Hispanics, and Asian Americans who have experienced oppression in the United States (Nieto \& Bode, 2008); 
11. Multiculturalism - the state or condition of emphasizing pluralism, confronting racism and biases, and seeing reality from a variety of perspectives (Nieto \& Bode, 2008);

12. Multicultural education - a field of study that supports (a) creating equal educational opportunities for students from diverse racial, ethnic, social-class, and cultural groups; (b) helping all students to acquire the knowledge, attitudes, and skills needed to function effectively in a pluralistic democratic society; and (c) teaching all students to interact, negotiate, and communicate with people from diverse groups in order to create a civic and moral community that works for the common good (Banks \& Banks, 1995);

13. Sheltered instruction—-strategies and techniques used to make content comprehensible for English language learners while they are developing English proficiency (Echevarría et al., 2008); and

14. Students of color-refers to students who have been labeled minorities such as American Indians, African Americans, Latinos, and Asian Americans who have experienced oppression in the United States (Nieto \& Bode, 2008).

Delimitations. The small sample size of this study was 15 teachers from one school. However, a sample of 30 participants is considered to be large enough for simple analyses (Bouma \& Ling, 2004; Creswell, 2008; Salkind, 2004). Due to the small sample used in this study, the results cannot be generalized to the school in which the research was conducted or to other schools with similar characteristics. The results of the study may eventually contribute to the development of a theory related to improving the 
professional development of teachers of English language learners. However, that theory will need to be tested with further research in additional contexts. 


\section{Chapter 2}

\section{Literature Review}

The literature review in this chapter consists of two components: a theoretical and empirical review of the literature and a conceptual framework. The theories reviewed in this paper serve the purpose of explaining the problem of study. The empirical studies lend support to the theories, propose alternate suppositions, and highlight the need for further study. The conceptual framework identifies obstacles to the quality instruction of English language learners, as well as the essential components for providing English language learners an equitable education.

\section{Multiculturalism}

Multicultural education, a philosophical concept based on the ideals of freedom, justice, equality, and equity (National Association for Multicultural Education [NAME], 2003), challenges the status quo (Nieto \& Bode, 2008). Multicultural education (a) recognizes the need to prepare students for an interdependent world and the important role schools can play in developing the requisite attitudes and values for a democratic society, (b) values cultural differences and affirms pluralism, (c) challenges all types of discrimination in schools and society by promoting social justice, (d) is a process that affects all aspects of a school's organization and policy so all students may achieve at the highest levels, (e) prepares students to work actively toward creating structural equality in organizations and institutions, and (f) encourages teachers and students to critically analyze relationships of power and oppression in their communities, society, and the world (NAME, 2003). 
Though the purpose of multicultural education is to provide equity and justice for all students through a comprehensive, ongoing process, the manner in which many educators understand and implement multicultural education is substantially less transformational (Gorski, 2006). Instead of using multiculturalism to promote social justice, many educators have redefined multiculturalism as a celebration of ethnic foods and festivals (Sleeter \& McLaren, 2009). Gorski asserted that while most attempts at implementing multicultural education are perhaps inconsistent with theory, the individuals attempting to implement multicultural education do so with good intentions and a sincere belief in equity. However, the incomplete and surface-level implementation of multicultural education has the effect of actually contributing to the inequities and injustices multicultural education was designed to eliminate (Nieto \& Bode, 2008).

The problem stems from the way current and future educators have been socialized into the teaching profession by the same messages and social structures that shape U.S. schools. By critically examining this socialization, educators can ensure they are not unintentionally contributing to the alienation and exclusion of others (Gorski, 2006). Perceptions of superiority are embedded in the structures of schools, the curriculum, the education most teachers have received, and the interactions among teachers, students, and the community (Nieto \& Bode, 2008). The root causes of social inequity, such as dominance or hegemony must be examined for teachers to provide an equitable education to all youth (Fine, 1997; Howard, 2003; McLaren, 2003). Moreover, Sleeter (1996) noted that if the analysis and understanding of the contributing factors of social inequity are superficial and incomplete, the approaches for addressing the inequity will be ineffective. 


\section{The Relationship Between Hegemony and Social Inequity}

Although few schools actually teach students the necessary skills to challenge inequity and pursue social justice for all, they often assert preparing students for active participation in a democracy as a principle educational goal (Nieto \& Bode, 2008). An analysis of hegemony and its role creating conditions for and perpetuating social inequity will help explain the chasm between the stated democratic beliefs of schools and the existence of a system that supports the inequitable education of a large population of poor and minority students.

Power, domination, control, supremacy, and authority contribute to creating a system of hegemony that maintains the status quo. Similarly, positivism and theoretical rationality legitimize both the existence of hegemony and the status quo. Characterized as a form of social administration and dominance (Giroux, 2001), positivism includes (a) the validation of cognitive thought through the experience of facts, (b) regarding physical science as a model of certainty and exactness, and (c) the belief that progress in knowledge is contingent upon the orientation toward physical science and exactness (Marcuse, 2002). In positivism, "Facts become separated from values, objectivity undermines critique, and the notion that essence and appearance may not coincide is lost in the positivist view of the world" (Giroux, 2001, p. 15).

The positivist perspective does not seem to allow for questioning of the conceptual systems that select, define, and organize what is perceived as factual (Giroux, 2001). How the conceptual systems were created, developed, and came to be viewed as the standard or norm is not examined either. This lack of analysis does not permit the understanding of the complex interaction of power, knowledge, and values; instead, it 
lends support to the assertion that objective knowledge exists and is inherently superior to other kinds of knowing (Friedman, 1978).

Marcuse (2002) contended the lack of critical analysis of society has its genesis in consumerism. The system of production creates goods and services, which subsequently market and impose the social system onto the people. That which is produced (e.g., mass transportation, communication, lodging, food, clothing, entertainment, and information) prescribes attitudes and habits that bind consumers to manufacturers, who subsequently connect consumers to society as a whole. Via indoctrination and manipulation, a false consciousness is achieved in which a way of life dedicated to consumption is perceived to be the ideal. Subsequently, a pattern of one-dimensional thought and behavior emerges in which ideas that differ from or transcend established thought are either rejected or refashioned into what already exists.

Technical rationality contributes to the false consciousness that consumerism relies upon to perpetuate itself. Defined by principles of control and certainty, technical rationality is used to control the environment through a process of deduction used to discover patterns among isolated variables. Knowledge is conceptualized as objective and neutral, as an entity that can be objectively tested. Empirical data are the focus of explanation and discovery, while other forms of knowledge are considered speculative and of less value (Giroux, 2001). Due to the belief that knowledge is neutral, objective, and exists in isolation, technical rationality does not question who legitimizes knowledge, how that knowledge is legitimized, or acknowledge that facts have to be interpreted by someone to be accessible (Friedman, 1978). 
Positivism and technical rationality contribute to singular perceptions of reality, which when legitimized contribute to the existence of privilege. Privilege allows people not to know, not to see, and not to act against unjust conditions (Howard, 2003). For example, individuals' perception of the significance of racism as a problem differs among Whites, who are the most privileged in U.S. society, and non-Whites. According to a recent CNN/Essence survey (Opinion Research Corporation, 2009), only 17\% of Whites believe racial discrimination is a very serious problem, contrasting the $55 \%$ of African Americans who do. A separate poll conducted by The New York Times and CBS News (2008) found racial discrimination to be a common experience among people of color with $68 \%$ of Blacks and $52 \%$ of Hispanics stating they had experienced a specific discriminatory incident. Informed by a review of national survey data about racial attitudes, Bobo (2001) asserted that Whites have a tendency to minimize the current persistence of discrimination, whereas Blacks, Latinos, and Asians recognize discrimination in most areas of their lives. Moreover, people of color are more likely to attribute patterns of discrimination to racial bias, while Whites are more likely to attribute problems to the individual's lack of effort or cultural values within the disadvantaged group. Sleeter (1994) contended that due to a strong belief in individualism, Whites tend to interpret racism as an individual belief versus an institutionalized system supported by a collective worldview. Acknowledging the existence of racism requires individuals to suspend their belief in rugged individualism and the U.S. as a meritocratic society (Tatum, 2003; Wise, 2009).

Rothenberg (2002) maintained that White privilege is the other side of racism. By examining White privilege, individuals can understand who benefits from racism and 
how. Once this understanding is reached, steps can be taken to dismantle racism on a personal and institutional level. A central aspect of White privilege is that Whiteness provides the norm or standard by which most other people and things are judged (Tatum, 2003). Whiteness is assumed and difference is understood in relation to it (i.e., a White person is not different from a person of color; rather the person of color is different). Ways of being that contrast Whiteness are often viewed negatively (Wildman \& Davis, 2002), benefiting those of the privileged group (i.e., because the non-privileged person is viewed as deviant, responsibility for adapting to the White norm falls upon his or her shoulders).

Another key factor in the power of White privilege is its invisibility. Many White people find it difficult to accept that White privilege exists and that they have benefitted from it (Rothenberg, 2005). Instead, success is often explained by superior intelligence and an individual's hard work or drive, rather than at least in part, his or her privilege. The focus on individual behavior obscures the existence of systems of privilege and power (Wildman \& Davis, 2005). White privilege directly supports the myth that everyone has access to power through individual resourcefulness (Wildman \& Davis, 2005). When racial privilege is unexamined, a sense of entitlement is produced. Achievements made more attainable by unearned privilege are viewed as fair compensation for an individual's effort. Therefore, when an individual's sense of entitlement is threatened, he or she often perceives a personal offense, rather than an arbitrary redistribution of privilege (Tatum, 2003). Accepting the existence of racism and its contributing factors, threatens many Whites' beliefs about society and their 
accomplishments in life. Therefore, talking about race is an uncomfortable topic that is often avoided in White communities.

Discussion about race in White communities is often absent, so Whites tend to think of racial identity as a characteristic that others have and something that is irrelevant to their experiences (Tatum, 2003). Fear prevents people from talking about race, lest they ask a naïve question or make an offensive remark, be uncomfortable with the level of honesty and vulnerability required to have such a discussion, and experience isolation or ostracism for challenging racism or bringing up topics that generate discomfort. Naming or discussing issues like the inequitable distribution of power and resources exposes realities and may disrupt people's belief systems (Fine, 2003).

One of the ways in which discussion is avoided and the status quo maintained is through the continued belief in the myth that knowledge is completely objective, neutral, and apolitical. Boler and Zembylas (2003) asserted that education explicitly and implicitly molds individuals to accept dominant cultural values. When such education is challenged (e.g., the dominant culture is questioned) it is considered propaganda. On the contrary, the exclusion of alternative views from the curriculum is often not considered a political act. Contrasting European western philosophy in which multiplicity and difference is negated, multicultural education asserts other truths exist and merit exploration (Nieto, 1995). However, the consequences of not naming or challenging the dominant cultural view in the areas of school administration, pedagogy, curriculum, and relationships between the school and community are most profound for low-income and minority students. Within the act of silencing, these students become alienated from the 
experiences they live, and ultimately from their educational development (Fine, 2003).

Ultimately, this silence is detrimental to all of us. Tatum (2003) noted,

As a society, we pay a price for our silence. Unchallenged personal, cultural, and institutional racism results in the loss of human potential, lowered productivity, and a rising tide of fear and violence in our society. Individually, racism stifles our own growth and development. It clouds our vision and distorts our perceptions. It alienates us not only from others but also from ourselves and our own experiences. (p. 200)

What is not said nor discussed, allows the status quo to continue. For that which is invisible cannot be fought, and as a result, privilege continues (Wildman \& Davis, 2005).

Resistance to cultural hegemony. Although privilege exists, it is also challenged by individuals in society. Teachers and students do resist cultural hegemony (Giroux, 2001). When issues of race, class, and gender are absent in school curriculum and classroom instruction, the dominant cultural view of U.S. society and school knowledge as inherently correct is legitimized. Avoiding issues of multiculturalism, communicates to students of color that school staff and the school as an institution do not acknowledge the existence of oppression or students' life experiences (Banks \& Banks, 2007). Refusing to learn as a form of political resistance in response to hegemonic content and methods of instruction becomes the outcome of schooling (Nieto \& Bode, 2008). Such resistance among students ranges from inattention in class, failure to do homework, negative attitudes toward school, poor relationships with teachers, misbehavior, vandalism, violence, and dropping out of school. In her research on 
students who had dropped out of high school, Fine (1991) found the two principle reasons for students' school desertion were (a) their disappointment with the promise of education and (b) a political stance of resistance. Classrooms in which teachers and students engage in a critique of power are sites of resistance (Anderson, Bently, Gallegos, Herr, \& Saavedra, 1998).

Opposing positivism and technical rationality's characterization of knowledge as objective, neutral, and singular; critical pedagogy questions the manner in which the dominant culture constructs knowledge and legitimizes particular forms of reality, while negating others (McLaren, 2003). Giroux (2001) explained critical theory is both a school of thought and process of critique. To create the conditions for social change, critical thinking and ongoing critique work in concert to question the claims of any theory and its portrayal of the world and the world as it actually exists. Hence, if the system is to become more equitable, each individual is responsible for his or her individual actions in society and for the system in which we participate (McLaren, 2003).

Giroux (1988) affirmed teachers must become transformative intellectuals if they are to educate students to become active, critical citizens. This can be accomplished by joining the political with the pedagogical and redefining schooling as both a struggle to define meaning and a struggle over power relationships. Instructing students from a multicultural education perspective is an example of the intersection of politics and pedagogy used to challenge the status quo of inequality. Giroux (1988) asserted, Within this view, critical reflection and action become part of a fundamental social project to help students develop a deep and abiding faith in the struggle to overcome economic, political and social injustices, and to further humanize 
themselves as part of this struggle. In this case, knowledge and power are inextricably linked to the presupposition that to choose life, to recognize the necessity of improving its democratic and qualitative character for all people, is to understand the preconditions necessary to struggle for it. (p. 127)

When education is an act of emancipation, and not of subordination, the conditions that create resistance are mitigated. Cummins (1996) maintained that when students' developing sense of self is affirmed and broadened through interactions with their teachers, they are more likely to increase their academic effort and participate actively in classroom instruction. The resultant learning motivates additional academic effort.

Because identity negotiation occurs throughout all educator-student interactions, educators must make concerted efforts to ensure students' identities and prior experiences are affirmed, not devalued. The importance of affirming students' identities cannot be understated. Communities and students of color are often given the message that success in school and society is contingent upon them abandoning any identification or connection they have with their culture and experiences. Students' prior experiences are seen as impediments to their academic growth, rather than the foundation upon which that development can be built (Cummins, 1996; Ogbu \& Simons, 1998). Ogbu and Simons (1998) emphasized the importance of a relationship of trust between minority students and their teachers characterized by teachers demonstrating with words and actions that they believe in their students, that their students' cultures are worthy of respect, and that success in school will leave their students' identities intact. Moreover, Ogbu and Simons (1998) advocated for teachers and students to discuss issues of 
resistance to school as a means of helping students critically evaluate their actions in response to instruction. Such discussion would also support teachers' understanding of the ways in which they may have structured learning activities that did not affirm students' prior experiences or their identities.

By devaluing the abilities and beliefs of students who are the most disempowered (i.e., minorities, poor, and females), the status quo is maintained in which schools, based on race, class, and gender sort, select, and favor students of the middle to upper classes. Inequality is produced through a system of meritocracy in which success is primarily attributed to individual characteristics while an individual's advantage and privilege is systematically ignored (McLaren, 2003). Furthermore, schools are part of power, politics, and history; they cannot be neutral or apolitical, as they are commonly perceived (McLaren, 2003).

The subsequent section of this paper describes an approach to teaching English language learners that does value their abilities and cultures while assisting them in meeting the same grade-level content standards that are expected of native-English speakers.

\section{Effective Instructional Approaches to Teaching English Language Learners}

My research study focused on the use of sheltered instruction, a technique used in content courses to teach grade-level objectives to English language learners. This is accomplished through the use of both content and language objectives and modified instruction, which includes the use of visual aids, vocabulary previews, cooperative learning, and the like. Sheltered instruction provides a bridge from English to Speakers 
of Other Languages (ESOL) classes, which focus on teaching the English language, to mainstream content classes (Echevarría et al., 2008).

Sheltered instruction is one component of a successful program for an English language learner. The international educational organization, Teachers of English to Speakers of Other Languages (TESOL) (1999) advanced recommendations for educating English language learners to acquire academic proficiency in English. TESOL (1999) identified the following predictors of English language learner academic success include (a) support in the student's native language, (b) family involvement in the child's education, (c) highly qualified teachers of English as a Second Language (ESL) who are trained in second language acquisition, (d) rigorous content standards coupled with high expectations, (e) a system of accountability using multiple measures for student assessment, (f) content-based instruction, (g) the alignment of curriculum, instruction, and assessment, (h) classroom teachers trained in ESL strategies, (i) commitment on behalf of the entire school to educate English language learner students, and ( $\mathrm{j}$ ) a learning environment that respects and honors the cultural diversity of students.

Sheltered instruction incorporates several of the components TESOL asserts predict academic success for English language learners. Additionally, both TESOL and proponents of multicultural education recognize the role and importance of including cultural diversity in the education of students. Second language acquisition (SLA) is referred to twice in the TESOL predictors of success. Both references discuss the importance of teacher training and SLA and ESL strategies, which apply SLA theory to classroom instruction. The next section of this paper will explain some of the principle theories of second language acquisition as they relate to sheltered instruction. 


\section{Sheltered instruction and related theories of second language acquisition.}

Learning a language is a long, complex endeavor influenced by multiple factors (e.g., learner and linguistic factors, learning processes, and instructional variables) (Brown, 2007). The Prism Model, as shown in Figure 1, helps explain the complexity involved in acquiring a second language (Collier \& Thomas, 2009). The model consists of four interrelated components that influence academic language acquisition: sociocultural, linguistic, academic, and cognitive. At the center of the model are the student's social and cultural experiences including that which occurred in the past, present, and future; as well as in all contexts (i.e., home, school, and community).

\section{Language Acquisition for School}

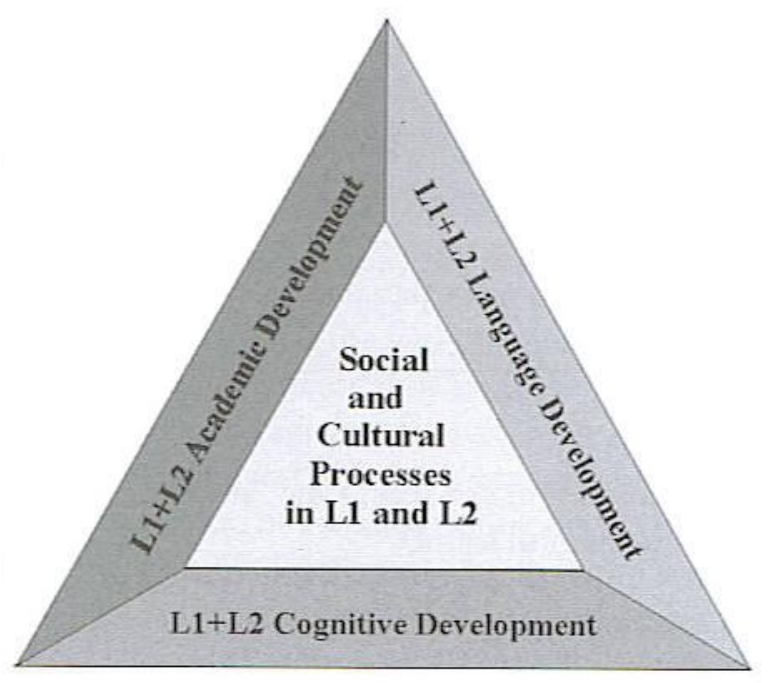

The Prism Model for Bilingual Learners

Figure 1. Prism model of second language acquisition. From Educating English Learners for a Transformed World (p. 34), V. P. Collier and W. P. Thomas, 2009, Albuquerque: Dual Language Education of New Mexico Fuente Press. Copyright, 2009 by Virginia P. Collier and Wayne P. Thomas. Reprinted with permission.

The social and cultural aspect of the model acknowledges the influence on language learning that sociocultural variables have such as an individual's self-esteem or 
the experience of being discriminated against by members of a community. The linguistic component of the model comprises the subconscious and conscious aspects of language development (i.e., the innate ability of humans to acquire oral language and the formal instruction of language in school, as well as the acquisition of writing). The high cognitive development of oral and written language in the student's first language significantly supports cognitive and academic success in the second language.

In their model, Collier and Thomas (2009) defined academic development as schoolwork in the subjects of language arts, mathematics, the sciences, and social studies. As students progress through the grades, the cognitive, lexical, and sociolinguistic demands increase dramatically. In that academic knowledge and conceptual development transfer between languages, offering academic classes in the students' first language, while teaching the second language through meaningful content during other times of the day, is most efficient and beneficial to the student.

The cognitive aspect of the model refers to the natural, subconscious process of constructing knowledge that occurs from birth through schooling and beyond. Collier and Thomas (2009) emphasized the importance of continuing cognitive development in the child's first language at the very least through elementary school. In the past, cognitive development was not sufficiently addressed by language teachers. Instead, language was simplified, while academic content was often reduced to basic skills. Cognitive development was also frequently neglected in the first language (Collier \& Thomas, 2009).

Because each component in the Prism Model influences the development of the other components, a student's overall development and success relies on program design 
and instruction that address each part of the model. At the center of the model is the sociocultural environment in which the student learns. Therefore, it is essential for educators to ensure the school environment is supportive to students and allows both their first and second language development to thrive (Collier \& Thomas, 2009).

The complexity of language acquisition is further illustrated upon examining the definition of linguistic competence. According to Bachman (1990), linguistic competence is divided into two types of knowledge: organizational and pragmatic. Organizational competence consists of grammatical and textual competence. Grammatical competence requires knowledge of vocabulary, morphology (word structure), syntax (analysis of sentence structure), and phonology (function and patterning of sounds) or graphology (systems of writing), while textual competence attends to the overall cohesion and organization of the discourse. Pragmatic competence consists of illocutionary and sociolinguistic competence. The illocutionary aspect focuses on sending and receiving messages, while the sociolinguistic aspect deals with politeness, formality, and other culturally related facets of language. Lastly, strategic competence involves the use of verbal and nonverbal communication strategies used to compensate for difficulties with communication.

Due to the complexity of language acquisition, becoming proficient in a language is a long-term process. Conversational fluency, also referred to as Basic Interpersonal Communication Skills (BICS), is context embedded (Cummins, 1979; 2000) and usually developed within two to three years, provided the learner had access to second language speakers and a social setting that encouraged natural linguistic interaction (Ovando, Combs, \& Collier, 2006). However, much of classroom, school-orientated language is 
context reduced and therefore more cognitively demanding (Cummins, 2000). This type of language, referred to as Cognitive Academic Language Proficiency (CALP), normally takes at least five years to acquire (Collier, 1995; Cummins, 1981; 2000; Hakuta, Butler, $\&$ Witt, 2000). Students who do not have prior schooling in their first language or access to native language development while learning the second language, may take seven to 10 years to develop CALPs (Collier, 1995).

The extent to which English language learners obtain academic English language proficiency influences their ability to adapt socially at school and is highly predictive of their overall academic success in the U.S. (Suárez-Orozco, Suárez-Orozco, \& Todorova, 2008). The importance of English proficiency, coupled with the difficulty and time involved in achieving it, underscores the importance of the use of second language acquisition research in designing and implementing educational programs in schools.

SLA research seeks to identify universal characteristics of second language acquisition and account for differences in the ways individuals learn a second language (Ellis, 2008). Such research provides insights into the process of acquisition, which teachers may subsequently use to make informed decisions about instruction (Center for Adult English Acquisition, 2009). The next section of this paper examines several principle theories of SLA as they relate to the learner's linguistic input, output, and the negotiation of meaning.

The role of input. Developing a high level of proficiency in a new language requires comprehensible input and targeted output. With the Input (Comprehension) Hypothesis, Krashen (2003) argued language is acquired only when the individual comprehends messages or receives comprehensible input. He asserted all learners 
progress along a natural order of language acquisition by understanding linguistic input that contains structures a little above their current level of competence.

Additionally, Krashen's (2003) Affective Filter Hypothesis stipulated learners acquire language when comprehensible input is present and learners' affective filter is lowered allowing input to reach them. An individual's affect consists of feelings, motives, needs, attitudes, and emotional states. If a learner is tense, anxious, bored, has a low self-esteem, or feels negatively toward a particular culture and its language, input may be blocked from reaching the language acquisition device or area in the brain responsible for language acquisition.

Critics of the Input Hypothesis asserted Krashen did not clearly explain the process that leads to learning, or clearly distinguish the difference between acquisition and learning (Færch \& Kasper, 1986; White, 1987). Færch and Kasper (1986) recognized that learners use contextual information and existing knowledge to understand utterances and that they may sometimes pay closer attention to the linguistic forms in messages. If learners are able to rely extensively on contextual knowledge and existing knowledge, they may pay little attention to the input's form and subsequently not acquire new knowledge. Færch and Kasper (1986) suggested that acquisition takes place only when learners experience a problem with comprehension, believe the problem is related to a deficit in their knowledge and not due to poor quality of input, and perceive these gaps to be their responsibility and not that of the interlocutor.

White (1987) challenged Krashen's assertion that comprehensible input causes second language acquisition. She argued that a substantial amount of acquisition occurs free of input. White referred to Corder's (1967) distinction between input and intake; 
input is language generated in the presence of learners, while intake is the language that is absorbed by learners and later remembered. Understanding input does not cause learners to unlearn certain types of overgeneralizations they make as they acquire a language. Learners require negative evidence such as corrective feedback. Additionally, for some linguistic structures, the failure to understand input may be a source of motivation for the learner to pay closer attention to the sentence structure to obtain clues about the utterance's meaning (White, 1987).

With its focus on providing students comprehensible input and time, support, and feedback related to students' targeted output (Echevarría et. al., 2008), the sheltered instructional approach incorporates aspects of Krashen's Input Hypothesis while taking into consideration the assertions of those who have responded to Krashen's theory with criticism. The sheltered instructional technique uses multiple means for making academic content understandable to English language learners. Sheltered instruction teachers tailor their speech to the proficiency levels of their students by attending to rate, enunciation, and complexity of their speech (e.g., vocabulary level, sentence structure, and idiom usage) (Echevarría et al., 2008). Academic tasks are explained clearly, and varieties of techniques are used to make concepts understandable. According to Echevarría and colleagues (2008) some principle techniques include (a) accompanying speech with the use of gestures, body language, pictures, and objects; (b) providing students with models of processes, tasks, and assignments; (c) previewing content for optimal comprehension by focusing students' attention on the information they are responsible for learning and using activities that activate students prior knowledge; (d) permitting students to use alternative forms for demonstrating understanding (i.e., hands- 
on activities); (e) exposing students to words, concepts, and skills multiple times; (f) presenting information in a well-organized format while ensuring students' knowledge is well-organized and accurate via the use of mental models (Jensen, 2005); and (g) using graphic organizers to visually demonstrate key points.

The role of output. Contrasting Krashen's (2003) assertion that linguistic output occurs too rarely for it to be of benefit to the learner, Swain (2005) declared output is as or even more important than input. Swain developed the Comprehensible Output Hypothesis, based on her assertion that Canadian language immersion programs had demonstrated comprehensible input alone was not sufficient to ensure learners achieved proficient levels of grammatical or sociolinguistic competence (e.g., using the appropriate register of language in both formal and informal settings). Production requires learners to attend not only to the message they wish to communicate, but also the form or grammatical structures used to communicate the message. Swain proposed three principle functions of output in SLA: (a) drawing the learner's attention to errors, (b) providing the learner an opportunity to try out language or test hypotheses that are forming about language, and (c) through speech and writing, affording the learner opportunities to reflect productively about language when interacting with peers.

Due to their lack of proficiency in English and the complexity involved in communicating information in English, English language learner students may not always be able to express the extent of their knowledge. Hence, it becomes more important for teachers of English language learners to respond by facilitating their students' communication of their thinking (Mohr \& Mohr, 2007). In addition to pacing lessons to fit students' proficiency levels and providing students sufficient wait time 
when asking questions, sheltered instruction teachers allow their students sufficient time for processing information (Echevarría et al., 2008). Direct instruction is limited to short increments, is followed by discussion time and small group and individual assignments. When students respond to teachers' questions during a sheltered instruction lesson, they are encouraged to elaborate their responses and avoid yes/no or one-word answers. Finally, the review of key vocabulary and content concepts remind English language learners of language that can be used to express their ideas. As teachers provide regular feedback to students about their language production, students improve their ability to communicate. As the correct use language and conceptual understandings are reinforced, misconceptions and misunderstandings are clarified and corrected (Echevarría et al., 2008). As part of the long-term process of second language acquisition, English language learners require substantial practice in developing the necessary linguistic competence to respond to questions, comprehend presented information, and participate in academic discussions.

The role of negotiating meaning. With his Interaction Hypothesis, Long (1996) emphasized the role of negotiation in facilitating language acquisition by directing the learner's attention to linguistic form. Negotiation of meaning occurs when a non-native speaker modifies her output as a result of receiving feedback about an error from a more advanced or native speaker. Long asserted the negotiation of meaning, especially that which causes the native or more competent interlocutor to adjust speech while interacting with the non-native speaker, facilitates language acquisition. Such acquisition occurs as connections are made between input; the capacities within the learner, especially selective attention; and output. Gass (2003) examined the relationship between 
communication and acquisition and concluded positive evidence or input consisting of well-formed sentences is necessary for learning. Production of language moves learners from focusing primarily on the meaning of language to a focus on the structure and formation of sentences. Output or language production also creates automaticity in learners in that a certain amount of practice is needed if learners are to produce language that is more fluent. Evidence of learning appears to occur because of the negotiation of meaning between non-native and native or advanced speakers of a language (Gass \& Varonis, 1994), in that corrected forms appear later on in non-native speakers' speech. Recognizing the importance of corrective feedback, the sheltered instruction model encourages teachers to provide students with regular feedback regarding their linguistic and conceptual output (Echevarría et al., 2008). Periodic reviews of language, vocabulary, and content allow teachers to provide students with specific academic feedback to clarify and correct any misconceptions they may have. Key vocabulary and content concepts are reviewed during a lesson, and particularly at the lesson's conclusion (Echevarría et al., 2008). A final review at the end of a lesson assists English language learners in assessing their own comprehension. Sheltered instruction teachers use students' responses during reviews to guide future instructional decisions (Echevarría et al., 2008).

Echevarría and her colleagues (2008) contended feedback, when supportive and validating, helps develop students' English proficiency. They provided an example of teachers using recasting or modeling to correct English usage by restating a student's response with the corrected grammatical forms. Lyster and Ranta (1997) studied the different types of feedback teachers give students regarding their linguistic errors and 
how students immediately responded to the correction. They found that while the teachers studied used recasts (i.e., correcting the mistake in a student's utterance and repeating the corrected version back to the student), the majority of the time; students were least likely to use the corrected form later on after it had been modeled. Students were more likely to use the correct form later if teachers had used elicitations, metalinguistic feedback, clarification requests, and repetitions, respectively (Lightbown \& Spada, 2006). Elicitations are the techniques teachers use to draw out the correct form from their students. These techniques include strategically pausing to allow students to fill in the blank, asking questions that cannot be answered with yes or no, and asking students to reformulate their utterance. In metalinguistic feedback, teachers use comments, information, or questions related to the correctness of the utterance to draw the student's attention to the existence of an error without explicitly telling the student the nature of the error. Clarification requests use phrases such as 'Pardon me...' and 'What do you mean by...?' to indicate to the student that the teacher has either misunderstood his/her utterance or there is a mistake. The goal is for the student, using the teacher's hints, to identify and correct the error. Repetition involves the teacher repeating a student's error, often adjusting his/her intonation, so the student's attention is drawn to the mistake.

In addition to receiving feedback from their teachers about the correctness of their utterances, students negotiate meaning with each other during sheltered instruction lessons (Echevarría et al., 2008). Sheltered instruction lessons use a variety of grouping configurations to support students in learning, discussing, and processing new information. Such lessons require students practice and use English to express and 
elaborate their ideas, opinions, and answers. Sheltered lessons are designed to foster meaningful interaction between students providing them practice with speaking and the negotiation meaning (i.e., asking and answering questions, clarifying ideas, giving and justifying opinions, and so forth).

Rigorous content standards coupled with high expectations. In addition to acknowledging the importance of second language acquisition theory, TESOL (1999) supported the use of content-based instruction that includes rigorous content standards paired with high expectations of English language learners. Content objectives are a key component of sheltered instruction lessons. Content objectives describe what students will learn to do, are used orally and in writing to simply state learning goals, and are tied to specific grade-level content standards (Echevarría \& Graves, 2007). Defining, displaying, and reviewing the content objectives with students assists the teacher and students in evaluating whether the objective was achieved at the end of each class and planning for future teaching (Echevarría et al., 2008). Like content objectives, language objectives are also clearly defined, displayed, and reviewed with students during a sheltered instruction lesson. Recognizing the complex and multifaceted aspects of language acquisition, language objectives in a sheltered instruction lesson vary in focus and may include receptive (listening and reading) and productive (speaking and writing) skills and process- and performance-orientated language use (Echevarría et al., 2008).

Once the content and language objectives have been established in a sheltered instruction lesson, various techniques are used to ensure an English language learners' academic and linguistic growth. Such techniques focus on (a) building students background knowledge by linking concepts to students' life experiences, (b) providing 
students with comprehensible input, (c) using learning strategies to support student understanding and critical thinking, (d) designing instruction for student-student interaction and student-teacher interaction which leads to frequent elaborated discussion and linguistic and conceptual feedback, and finally (e) providing students with activities to practice, apply, and review what they have been learning (Echevarría et al., 2008). By expecting English language learners to meet high academic standards and scaffolding their learning with sheltered instructional techniques, English language learners can reach

grade-level standards. Conversely, when students who are attempting the difficult task of learning both language and content are provided low levels of instruction and poor quality linguistic and content interactions, which is often the case, the result is poor academic achievement (Mohr \& Mohr, 2007).

\section{Sheltered Instruction and Multicultural Education}

Aligned with TESOL's recommendations and characteristics of multicultural education, creating a learning environment that respects and honors the cultural diversity of students is a critical component of the sheltered instruction approach. Effective teachers of sheltered instruction consider their students' cultural backgrounds and learning styles when planning for and delivering instruction (Echevarría et al., 2008). For example, during the building background component of a sheltered lesson, the teacher explicitly connects the concepts of focus to students' background experiences.

Throughout the lesson, teachers provide comprehensible input in multiple ways using a variety of techniques to make content concepts clear. This supports students of various learning styles as they learn the lesson concepts (Echevarría et al., 2008). 
In addition to creating a learning environment that honors and respects cultural diversity, sheltered instructional lessons support multicultural education at the classroom level by structuring learning so that English language learners are successful at meeting high standards, contrasting the remedial type of instruction that is frequently offered to English language learners (Collier \& Thomas, 2009). Characteristics of sheltered instructional lessons include (a) content objectives selected from grade-level academic standards, (b) meaningful activities used to integrate lesson concepts and provide students practice in all four language domains, (c) concepts linked to students' background knowledge, (d) an emphasis on key vocabulary, (e) scaffolding techniques used throughout the lesson to support student understanding, (f) students grouped to interact and discuss lesson concepts with academic language, and (g) a review of key vocabulary and content concepts at the end of each lesson (Echevarría et al., 2008).

Establishing a classroom environment in which students' affective needs are met is critical to the success of a sheltered instruction lesson and particularly important for students who have not been successful in school. A sheltered instructional lesson addresses students' affective needs in multiple ways. In addition to providing students with substantial language and problem-solving practice, the use of pairs and small groups creates a more comfortable, supportive learning environment. In sheltered instructional lessons, an emphasis is placed on students working cooperatively versus competing against one another (Echevarría et al., 2008). Students' English proficiency and academic levels determine the pacing of lessons. Additionally, assessments are adapted to accurately measure English language learners' knowledge while maintaining high 
standards. Finally, student responses are validated with paraphrasing, a technique that supports students in feeling comfortable and able to take risks in the classroom.

School success relies upon, but is not limited to, classroom learning and the ability to perform technical skills competently (Stanton-Salazar, 1997). Knowledge of the cultural capital and belief systems embedded in mainstream institutions, like schools, is also central to students' academic success. Within the contexts of the home and community, dominant culture children are socialized at an early age into mainstream institutional life. This socialization includes knowledge of (a) socially acceptable ways of using language and communicating, (b) academic subject areas, (c) how bureaucracies operate, (d) networking behavior, (e) technology, (f) labor and educational markets, and (g) how to solve problems related to school, making sound decisions, and reaching personal and collective goals (Stanton-Salazar, 1997). Being able to decode the mainstream system is an essential component of poor and minority students' academic success. To thrive within the system, poor and minority students need to engage in the academic process communally, rather than individualistically, as they continue to maintain their connection to systems of support within their families and communities (Stanton-Salazar, 1997). For individuals from socially marginalized groups, the maintenance of nondominant cultural capital supports their sense of belonging, connection, and identity (Carter, 2005). Their challenge is to manage living in multiple worlds (Stanton-Salazar, 1997). However, the responsibility for adapting to multiple contexts should not fall solely on the shoulders of nondominant culture students. Rather, both students and educators must embrace multiple cultural capitals (Carter, 2005). 
Cummins (2000) stressed the importance of educators and students facing issues of equity and power together. He asserted:

...the negotiation of identity in the interactions between educators and minority students is central to students' academic success or failure...These interactions reflect both the organizational structures that have been implemented (first language promotion or first language suppression) and the ways in which educators have defined their roles with respect to the purposes of education in general and culturally diverse students and communities in particular...This perspective clearly implies that in situations where coercive relations of power between dominant and subordinated groups predominate, the creation of interpersonal spaces where students' identities are validated will entail a direct challenge by educators (and student) to the societal power structure. School reform efforts that fail to challenge coercive relations of power rarely succeed because they do not address the causes of school failure. (p. 258) Multicultural education, when implemented correctly, challenges coercive relationships of power (Nieto \& Bode, 2008). Implementing multicultural education in schools requires current practices to undergo substantial change (Banks \& Banks, 2009). Reform must occur related to (a) relationships of power, (b) communication between teachers and students, (c) the culture of the school, (d) the curriculum, (e) extracurricular activities, (f) assessment, (g) grouping practices (Banks \& Banks, 2009), and (h) attitudes toward minority languages (Romaine, 2009). In short, the school's institutional norms, social structures, cause-belief statements, values, and goals all must be transformed and reconstructed. 
Such extensive change will require educators to transform their beliefs about teaching and learning as well as their practice as they learn new ways of communication, thinking, reflection, and teaching. This requires high-quality professional development that incorporates adult learning theory in its design and delivery. The next section examines the role of teacher beliefs in educational practice, the core principles of adult learning theory, and best practices in the professional development of teachers.

\section{Relationship Between Teacher Beliefs and Instructional Practice}

According to Borko and Putman (1996), learning is "an active, constructive process that is heavily influenced by an individual's existing knowledge and beliefs and is situated in particular contexts" (pp. 674-675). Beliefs, defined as psychologically held understandings or premises about the world that are believed to be true, significantly influence how and what preservice teachers learn (Richardson, 1996). As students enter preservice teacher education programs, they bring with them the beliefs about teaching, learning, and subject matter they have acquired throughout their experiences as students (Griego Jones, 2002). Acknowledging the influence preservice teachers' beliefs have on the education of English language learners, Griego Jones (2002) asserted:

As extensive as it is, the body of knowledge about effective practices for second language learners is useless if it doesn't connect with the preservice teacher's beliefs about second language learning. There has to be some readiness within the preservice teachers themselves to receive the knowledge and do something with it. (p. 45)

Because preservice teachers often already have clear beliefs about concepts and ideas (e.g., group learning, assessment, and diversity) discussed in preparation courses, they 
tend to assimilate what is being taught with their preexisting schema. This can create a barrier to developing deeper conceptual understanding (Hammerness, Darling-Hammond, \& Bransford, 2005).

In terms of my hypothesis, I believed teachers who had not developed an understanding of the importance of multiculturalism in student learning would be more likely to assume incorrectly that instruction appropriate for native-English speakers would also be effective with English language learners. Therefore, these teachers would be less likely to use sheltered instructional techniques with their English language learner students.

Programs that change beginning teachers' understanding of teaching and learning use their students' initial beliefs about teaching as catalyst for surfacing and confronting misconceptions via structured discussions and guided observations of classrooms (National Academy of Education Committee on Teacher Education, 2007). When preservice teachers are not engaged in the critical examination of their beliefs in the context of compelling alternatives or assisted in the development of powerful images of good teaching and strong professional commitments, their entering beliefs will continue to influence their ideas and practice (Feiman-Nemser, 2001). Ultimately, to become effective, teachers must learn to integrate ways of thinking, knowing, feeling, and acting into an ethical and responsive teaching practice (Feiman-Nemser, 2008). Accomplishing such a task requires quality professional development that integrates theories of adult learning. Such professional development is discussed in the subsequent section. 


\section{Adult Learning Theory}

Explaining how adults learn has been approached from numerous perspectives, including, but not limited to andragogy, cognitive theory, critical theory, developmental psychology, elemental and holistic models, philosophy, and spirituality

(Knowles, Holton, \& Swanson, 2005; Mackeracher, 2004; Merriam, 2008). Aspects of the various approaches are included in Vella's (2002) principles for effective adult learning and in the andragogy described by Knowles and colleagues.

Core adult learning principles. Andragogy is defined as a set of essential adult learning principles that apply to all adult learning situations (Knowles et al., 2005). The principles include the (a) learner's need to know, (b) self-concept of the learner, (c) prior experiences of the learner, (d) individual's readiness to learn, (e) individual's orientation to learning, and (f) individual's motivation to learn. Adult learners benefit from knowing why they should study a particular topic before beginning to learn it. Furthermore, adults develop readiness to learn that which they believe will help them cope effectively with real-life situations. Similarly, adults' orientation to learning is life-centered. Adults are motivated to learn what they perceive will help them perform tasks or resolve problems they confront in their daily lives.

In his work with adults in developing countries, Freire (2003) developed a process he referred to as conscientizaçāo or conscious raising. In this process, oppressed people become aware that their worldview and place within the world is influenced by social and historical forces, which work against their interests (Tennant, 2006). According to Freire (2003), all authentic education examines thinking. An individual cannot think for others, nor can others think for him or her. Through conscientizaçāo, people leave behind their 
status as objects and use praxis to become subjects in the struggle against their own dehumanization.

In his work with literacy programs in Brazil, Freire and his colleagues avoided the use of a mechanistic literacy programs and instead taught adults to read within the context of raising their consciousness about causality and developing critical thinking (Freire, 1974). Rather than being told why it was important for them to learn to read, the adults in these literacy programs experienced how learning to read and write changed their thinking and provided them with tools to act upon the injustice they experienced in their lives.

Being responsible for their own decisions and lives is part of adults' self-concept (Knowles et al., 2005). Inherent in that self-concept is the need for others to perceive and treat adults as capable of self-direction. Situations in which others seem to impose their will tend to be met with resistance and resentment from adults. Learning experiences, which encourage adults to be self-directed learners, support adults' self-concept. Tough (1982) investigated the intentional change, a form of self-directed learning, of 330 adults in Canada, England, and the United States. The adults ranged in age from 25-85, were predominantly White (73\%), and of the middle to upper class (72\%). Using interviews Tough explored how people chose to change, what they tried to change, and who or what helped them change. The researcher found most men and women were successful at achieving the changes they selected, with an average of $80 \%$ of the study's participants achieving the changes they had chosen. Tough's study appears to lend support to the assertion that adults are generally successful self-directed learners. However, it is important to acknowledge the results of Tough's study are based on a sample of 
predominantly White, middle to upper class adults whose approach and style of learning is specific to their culture and class (Brookfield, 1985).

Because adults have lived longer, they have a larger amount and different quality of experience than younger people (Knowles, et al., 2005). Furthermore, adults define who they are based on their experiences. In learning situations in which adults' experiences are ignored or devalued, adults will perceive this as a rejection of their experience as well as their person.

As a group, adult learners are quite heterogeneous in terms of their background, learning style, motivation, needs, interests, and goals. This range and quantity of experience provides adult education classes a rich resource for learning. However, as individuals accumulate experiences, they tend to develop mental habits, biases, and presuppositions that create resistance to new ideas and alternative ways of thinking. Transformative Learning Theory contends individuals change their thinking through a process of critically reflecting previously unexamined assimilated assumptions, beliefs, values, and perspectives (Mezirow, 2000). The process can be brought about by a single dramatic event, a series of almost imperceptible cumulative events, an individual's intentional effort to change his or her life, or the natural developmental progression of maturing (Cranton, 2006). Once an individual has become critically reflective of his or her assumptions, engaging in discourse with others supports the individual's ability to view an issue from multiple perspectives. As individuals become more reflective and open to other's perspectives, their capacity to imagine alternative contexts for understanding is enhanced (Mezirow, 2000). 
Finally, adults respond to external motivation such as promotions, but are more significantly influenced by internal pressure such as self-esteem and quality of life (Knowles, et al., 2005). According to Wlodkowski (1999),

All adults want to make sense of their world, find meaning, and be effective at what they value. This is what fuels their motivation to learn. They key to effective instruction is to evoke and encourage this natural inclination- the propensity in all adults, whatever their background or socialization, to be competent in matters they hold important. (p. xi)

In an investigation of the factors associated with adults' time spent in learning activities, Livneh and Livneh (1999) surveyed adults in educational programs and found selfmotivated learning (i.e., being internally motivated to obtain additional knowledge), to be the greatest predictor of the amount of time spent in professional growth activities. External motivation and participants' educational level (i.e., having at least a bachelor's degree) were secondary predictors of the amount of time adults spent in professional learning activities.

Although andragogy serves the purpose of providing educators a guide for teaching adults, a limited number of well-designed empirical studies actually support andragogical theory (Tennant, 2006). Andragogy has also been criticized for portraying all adult learning as universal and ignoring the context in which the learning occurs (Merriam, Caffarella, \& Baumgartner, 2007). Chief concerns with andragogy include (Sandlin, 2005) (a) the assumption that education is value neutral and apolitical, (b) the promotion of a generic adult learner as universal with White, middle class values, (c) disregard for other ways of knowing and subsequent silencing of other voices, (d) a lack 
of awareness about the relationship between the self and society, and (e) the reproduction of inequalities and support of the status quo. According to Griffin (1991), adult learning lacks a "sense of the historical, economic, and cultural forces that shape the possibilities for and the meaning of individual growth and transformation" (p. 268). Consequently, educators must understand how to design learning in their classrooms in such a way that culturally diverse students are not required to sacrifice their cultural identities if they are to learn (Alfred, 2003).

The use of dialogue in instruction is one means of giving voice to individuals who are often silent, acknowledging other ways of knowing, and understanding the uniqueness of each learner in a particular context. Vella (2002) maintained that adult learning is best accomplished through dialogue, and proposed 12 learning principles and practices for beginning, maintaining, and nurturing dialogue. Freire (2003) also emphasized the importance of dialogue and its application:

Only dialogue, which requires critical thinking, is also capable of generating critical thinking. Without dialogue there is no communication, and without communication there can be no true education...Dialogue further requires an intense faith in humankind, faith in their power to make and remake, to create and re-create, faith in their vocation to be more fully human (which is not the privilege of an elite, but the birthright of all). (p. 90, 92-93) Vella's (2002) approach to adult learning consisted of 12 principles: (a) a needs assessment that involves the learners in identifying what is to be learned; (b) safety in the environment and process used for learning and teaching; (c) sound relationships between the teacher and learners and among the learners; (d) sequencing content to begin with 
relatively simple tasks that become progressively complex as well as reinforcing facts, skills, and attitudes in engaging ways until they are learned; (e) praxis or learning by doing coupled with reflection; (f) respect for learners as decision makers; (g) designing learning activities to include the cognitive, affective, and psychomotor aspects of learning; (h) clarifying the immediate usefulness of the learning; (i) clear roles and role development that support engagement in dialogue; (j) teamwork and use of small groups, (k) engagement of the learners in what they are learning; and (l) accountability of the teacher to the learners in designing activities that meet the established objectives, as well as learners' accountability to each other and to their teacher.

Concepts of quantum physics such as relatedness, a holistic perspective, duality, uncertainty, participation, and energy are evident in Vella's (2002) approach to adult learning. Each of the 12 principles and practices are related to the others. The holistic perspective asserts the whole is more than the sum of its parts; the learner brings his or her entire life experience to the learning, which in turn affects the content and process used for teaching. Dualistic thinking encourages dialogue through the use of open questions and dialectical thinking. As theories are applied to new contexts, they are adapted and changed. Certainty is not a given; adults' education cannot be static and designed from the same mold if it is to be effective. Learners' participation is incorporated into the needs assessment, their role in making decisions, the inclusion of cognitive, affective, and psychomotor aspects of learning, and through the use of dialogue in their education. Contrasting certainty, participation recognizes the effect of context and culture on a learner's perspective. Learning requires energy. Numerous principles create and maintain the energy level of adult learners (e.g., the establishment of 
a safe context for learning, effective sequencing and reinforcement of content, the use of praxis in learning, and participant decision making).

The range of theories and perspectives on adult learning provide the adult educator with a foundation for improved instructional design and outcomes (Knowles, et al., 2005). Factors such as the learner's self-concept and motivation, relevancy of the learning task, relationship between the teacher and learners, and the role of critical thinking in the teaching and learning influence the extent of the learner's growth. Andragogy is an important component of well-designed professional development for teachers. The other essential components of quality staff development, as well as the unique needs of English language learner teachers, are described in the next section.

Effective practices in the professional development of teachers. As discussed in Chapter 1, quality teaching has a significant effect on the academic gains of students. The professional learning of teachers and administrators is a critical factor in determining instructional quality. Additionally, district structures and school cultures play an essential role in determining the quality of professional learning experienced by teachers and principals (Sparks, 2002).

High quality staff development has been defined as that which (a) focuses on deepening teachers' content knowledge and pedagogical skills; (b) includes opportunities for dialogue, practice, research, and reflection; (c) is embedded in educators' work and takes place during the school day; (d) is sustained over time with adequate support systems (e.g., observation, modeling, coaching, and problem solving); (e) is founded on a sense of collegiality and collaboration among teachers and between teachers and principals in solving important problems related to teaching and learning; and (f) uses 
andragogical instruction (Darling-Hammond \& McLaughlin, 1995; Díaz-Maggioli, 2004; Sparks, 2002).

If the essential purpose of professional development is to advance the use of effective instruction that results in learning gains for all students (Díaz-Maggioli, 2003), such professional development must be specifically designed to instruct teachers of English language learners in the specific knowledge and attitudes essential to the teachers' and their students' success (Clair \& Adger, 1999). Teachers of English language learners must have the following knowledge, skills, and attitudes to educate their students effectively: (a) a solid understanding of language acquisition, communicative competence, and the role first language and culture play in language learning and academic achievement; (b) content area expertise, in addition to being language teachers who are able to make academic content comprehensible to students; (c) the perception of English language learners as having resources relevant to learning and the ability to learn, rather than equating limited English proficiency and academic skills as deficiencies; (d) proficiency in planning, implementing, and managing instruction, as well as the proper methods and techniques for assessing language growth; and (e) an understanding of teaching as a political and ethical act that requires educators to critically examine personal beliefs about race, culture, and language, to be able to identify and challenge inequitable school practices (Clair, 1993; Friedman, 2002; TESOL, 2003; Téllez \& Waxman, 2005; Villegas \& Lucas, 2002; Walqui, 1999). 


\section{Conceptual Framework}

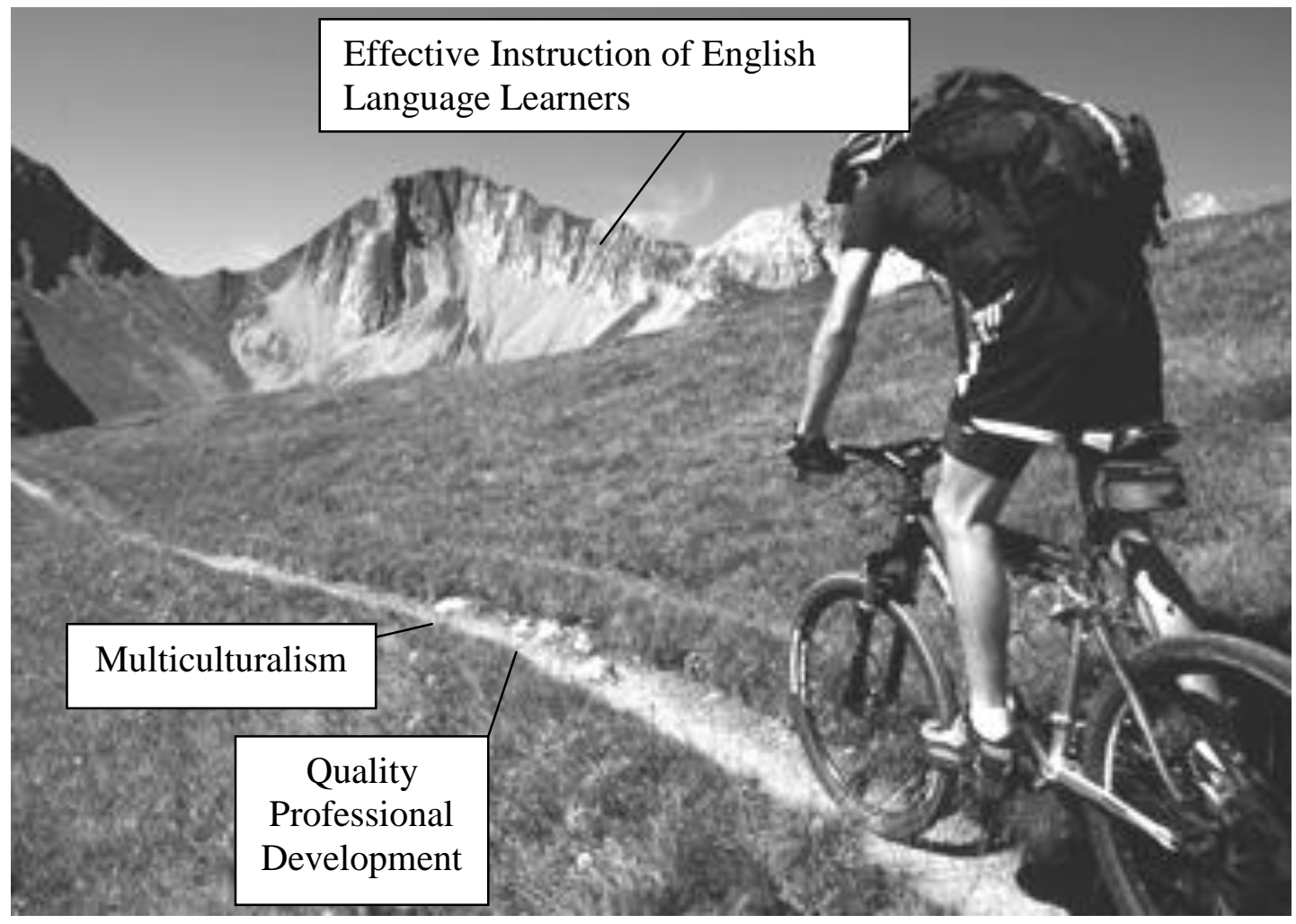

Figure 2. Path to educating English language learners successfully.

The model for successfully educating English language learners identifies the barriers this population often faces related to their education, as well as the factors that ensure their linguistic and academic success. Quality professional development and the use of multiculturalism to guide instructional practices provide a means of reaching the goal of effective instruction of English language learners.

Multiculturalism in this model refers to educators' use of multicultural education to value students' diverse cultures, affirm pluralism, and critically analyze relationships of power and oppression to challenge discrimination while promoting social justice (NAME, 2003). Related to multiculturalism, educators' beliefs about teaching and learning can support or undermine English language learners' education. Several tenets 
of multiculturalism support the perception of teaching as a political act. Teachers and students are encouraged to analyze relationships of power and oppression in their community, society, and the world and respond by working actively toward establishing equity. Researchers found the belief that all students could be successful learners and their communication of that belief to students was a common element among effective teachers in urban schools (Delpit, 1988; Gay, 2000). Perceiving teaching as a political act and believing all students to be capable of learning have the effect of supporting English language learners' academic and linguistic success. Finally, in that a large number of teachers of English language learners do not feel prepared to teach this population of students (Lewis et al., 1999), they will need additional professional development. To address the instructional needs of their English language learner students, teachers' professional development must include the skills, knowledge, and attitudes required to instruct both academic content and develop language proficiency (Clair \& Adger, 1999).

The dirt path is a means of reaching the mountain. However, the status quo has the ability to erect barriers along the path of multiculturalism and quality professional development, which can prevent or at least slow the bicyclist or educator's success in reaching the mountain (i.e., goal of providing English language learners with instruction that meets their academic and linguistic needs). In society, the status quo provides the overwhelming rationale for how decisions are made, which decisions are made, and who is involved in the decision making (Giroux, 2001). If the bicyclist is to reach the mountain, he or she must respond to the barriers that are presented on the path. For example, a decision to ride through a pothole similar to the action taken upon seeing the 
last pothole can result in a flat tire. Unlike the previous pothole, this one had a sharpedged rock embedded in the dirt. The bicyclist failed to acknowledge how the two potholes were actually distinct and did not respond appropriately. By valuing diverse cultures and perspectives, multicultural education challenges the status quo (Nieto \& Bode, 2008) to acknowledge that mainstream U.S. culture is not the standard to which all other cultures must assimilate, rather the expectation is that individuals must develop the ability to see difference and respond appropriately.

In addition to potholes and flat tires, the bicyclist could face pedaling against a strong wind. Professional development as most teachers and principals experience it is often unfocused, insufficient, and irrelevant to the daily challenges faced by educators (Sparks, 2002). When professional development is that ineffective, it does not have the effect of supporting the educator in learning how to teach effectively English language learners. Conversely, the poor quality professional development could have the effect of slowing down the educators' progress toward learning to instruct English language learners effectively.

\section{Relationship Between the Literature Review and Proposed Study}

Some believe the U.S. has established a public educational system in which all are

provided the equal opportunity to learn and succeed academically. Others argue that such a system, built on the belief that all are capable of succeeding, does not exist (Darder, 1991; McLaren, 2003). High school dropout rates, quality of instruction indicators, national content area assessment results, and school funding allocations along ethnic, racial, and socioeconomic lines attest differences exist among several populations, 
resources are not necessarily distributed to those who need them the most, and privilege is consistently provided to some while denied to others.

Zeichner (2003) contended that three key practices are required for all students to achieve high standards: (a) educators' high expectations of students, (b) culturally responsive instruction, and (c) the use of instructional strategies for making meaning (e.g., use of dialogue in instruction and a rejection of the transmission model of education). Studies have shown many teacher education students have lower expectations of some groups of students, even when presented with evidence that demonstrated the students' ability to meet higher standards (Goodlad, 1990; Pang \& Sablan, 1998). Additionally, Valli (1996) found many teacher education students had negative attitudes about students from racial, ethnic, and linguistics groups different from their own. In that the majority of public school teachers are White, female, and middle class (National Education Association, 2003; Strizek, Pittsonberger, Riordan, Lyter, \& Orlofsky, 2006), and the student population continues to become more culturally, linguistically, ethnically diverse, and poor (Garofano \& Sable, 2008; Lindholm-Leary, 2000; Tang, Sable, \& Hoffman, 2009); the need for culturally responsive instruction becomes ever apparent.

Most teacher education programs have a limited amount of substantive programs or courses that address diversity (Gollnick, 1995). Moreover, student teachers from such programs significantly struggled in classroom placements in which the students were culturally and linguistically different from them (Birrell, 1994; Goodwin, 1994; Weiner, 1990). Because most teacher education programs do not adequately prepare teachers to educate students of color, it is necessary for school districts to provide teachers with on- 
site professional development. Quality professional development must expand teachers' understanding of the complexity of ethnic groups and how race, ethnicity, language and social class influence student behavior (Banks et al., 2005) and incorporate best practices in its design (e.g., is sustained over time with adequate support systems and includes dialogue, practice, research, and reflection for its participants) (Darling-Hammond \& McLaughlin, 1995; Díaz-Maggioli, 2004; Sparks, 2002). For teachers of English language learners, professional development must be specific to the knowledge, skills, and attitudes such teachers need to ensure their linguistically diverse students' needs (Clair \& Adger, 1999).

Throughout this paper, the case has been made for educators to frame their work with students from a multicultural perspective whereby politics and pedagogy intersect to challenge the status quo of inequality. Yet, the majority of teacher education programs do not train teachers to do this (Gollnick, 1995). Moreover, recommendations in the staff development literature are insufficient for guiding the professional changes necessary for teachers to become adept at culturally relevant instruction (Zeichner \& Hoft, 1996). My study focused on the long-term sheltered instruction staff development of teachers of English language learners at a school with a large English language learner population. The study examined the relationship between teachers' attitudes and beliefs about instructing diverse students and their use of the sheltered instructional approach. The results of the study may encourage the school district and research site within to approach professional development differently, so that the information and pedagogical techniques presented during the professional development sessions is successfully applied in teachers' classrooms. 


\section{Chapter 3}

\section{Methodology}

An explanatory mixed method design was used to conduct this study. In explanatory mixed method research, quantitative and qualitative data are collected in two phases: Phase 1 involves the collection of the quantitative data, which are the priority of the study, while Phase 2 focuses on the qualitative data (Creswell, 2008). The quantitative data and results establish a general idea of the research problem; further analysis using qualitative data collection serves the purpose of refining, extending, or explaining the general idea (Creswell, 2008). Mixed method research has strengths that quantitative and qualitative research do not have when used separately. Quantitative research has been criticized for not allowing participants' voices to be heard directly and for lacking understanding of the context of the study. When data are reported in quantitative studies, the researchers are in the background yet their own personal biases and interpretations are often not discussed. Qualitative research has been challenged because of the personal interpretations of the researcher may result in bias and the difficulty of generalizing findings to a large group because of the limited number of participants studied (Creswell \& Plano Clark, 2007).

Previously, I argued against positivism and described its role in maintaining the status quo. When results are accepted without a process of examination and questioning, quantitative research can support positivism. However, I chose to include quantitative research in my study because of the contribution it makes toward obtaining a general understanding of the problem and because I examined and questioned the quantitative 
results via qualitative data collection and analysis. Further, the use of quantitative methods lends credibility to this study in that quantitative research is widely respected.

Explanatory correlational research was used to examine the relationships between the Demographic Questionnaire and the quantitative measures used in the study: the Multicultural Efficacy Scale (MES), the Sheltered Instruction Observation Protocol (SIOP), and question six of the Classroom Instruction Questionnaire (CIQ); and the qualitative section of the CIQ and the semi-structured interview, a qualitative instrument.

\section{Research Design}

During Phase 1 of this explanatory correlation study, quantitative data were collected at one point in time, with at least two scores per variable being obtained for each individual in the group. Participants' data were analyzed as a single group and in subgroups based on their total MES and SIOP scores. Correlational tests were conducted on the Demographic Questionnaire variables, MES, SIOP, and the results of question six of the CIQ. In my study, I reported the use of the correlation statistical test in the data analysis and used the statistical results to make interpretations or draw conclusions (Creswell, 2008).

Phase 2 of the study involved the collection of the qualitative data in the study (i.e., the first five questions on the CIQ and semi-structured interview responses). The collection of the qualitative data served the purpose of elaborating upon the quantitative results. Participants' qualitative responses offered distinct perspectives on the topic and more detailed, specific information not evident in the quantitative results.

The process used to conduct the study ensured that the data analyses were matched to the study's explanatory mixed method design. The design began with the 
collection and analysis of the quantitative data (Creswell \& Plano Clark, 2007). The Pearson Product-moment Correlation was used to determine any relationships between variables on the quantitative measures (i.e., the MES, the SIOP, and question six of the CIQ) and variables on the Demographic Questionnaire and quantitative measures. Next, the qualitative data were collected and analyzed (Creswell \& Plano Clark, 2007). Based on the quantitative data results and the responses to the CIQ, six individuals were selected for interviewing. Once the interviews had been completed and analyzed, the relationship between all of the data were examined as well as the original thinking behind the hypothesis that there was a relationship between participants' scores on the MES and SIOP.

\section{Context and Access}

The research site was a middle school of approximately 600 students, with $57 \%$ of the student population being English language learners. Of the 38 teachers at the school, approximately 20 had participated in sheltered instruction training. Access to the school was facilitated by my position there as a Teaching and Learning Coordinator. In this position, half of my time is spent coordinating the school's English to Speakers of Other Languages (ESOL) program, while the other half of my time is dedicated to coaching teachers. The middle school principal and district superintendent agreed to the study.

\section{Participants and Selection Process}

Because the school had only 38 teachers, I oversampled the population by initially asking all teachers to participate in the study. My plan was to follow the initial sampling with maximal variation sampling, a type of purposeful sampling in which the researcher samples individuals who differ on a particular characteristic (Creswell, 2002) (i.e., 
teachers who had and had not participated in sheltered instruction training). However, only 15 teachers agreed to participate in the study. Because the number of teachers who expressed interest in the study was limited, all were accepted as participants for the study. Consequently, convenience sampling was used in this study. Because the sample consisted of individuals who were not systematically selected for the study, the results cannot be generalized with confidence to the entire teaching population of the school (Creswell, 2008). Additionally, 30 participants in a study would be needed to provide a large enough sample for simple types of analyses (Bouma \& Ling, 2004; Creswell, 2008; Salkind, 2004). Therefore, this study lends itself to being characterized as a pilot study. Results from the Demographic Questionnaire show the majority of the study's participants were female, Christian, and between 41 to 60-years-old. All participants had traveled outside the U.S., with the majority being bi or multilingual and born in the U.S. None of the participants was physically disabled (see Table 2). 
Table 2

Personal Background of Participants $(N=15)$

\begin{tabular}{|c|c|c|}
\hline Variable & Frequency & Percent \\
\hline \multicolumn{3}{|l|}{ Gender } \\
\hline Male & 4 & 26.7 \\
\hline Female & 11 & 73.3 \\
\hline \multicolumn{3}{|l|}{ Age of participants } \\
\hline 21-40-years-old & 4 & 26.7 \\
\hline 41-60-years-old & 11 & 73.3 \\
\hline \multicolumn{3}{|l|}{ Birthplace of participants } \\
\hline In the U.S. & 9 & 60.0 \\
\hline Outside of the U.S. & 6 & 40.0 \\
\hline \multicolumn{3}{|l|}{ Race of participants } \\
\hline Caucasian & 8 & 53.3 \\
\hline Non-Caucasian & 7 & 46.7 \\
\hline \multicolumn{3}{|l|}{ Religion of participants } \\
\hline Christian & 11 & 73.3 \\
\hline No religion & 4 & 26.7 \\
\hline Physical disability of participants & 0 & 0.0 \\
\hline \multicolumn{3}{|l|}{ Linguistic status of participants } \\
\hline Monolingual & 6 & 40.0 \\
\hline $\mathrm{Bi}$ or multilingual & 9 & 60.0 \\
\hline Travel outside of the U.S. & 15 & 100.0 \\
\hline
\end{tabular}

The highest educational degree of the participants and their parents are displayed in Figures 3-5. While a Master's degree was the highest degree obtained by the participants, a high school or general equivalency degree was the highest degree achieved by the participants' mothers and fathers. 


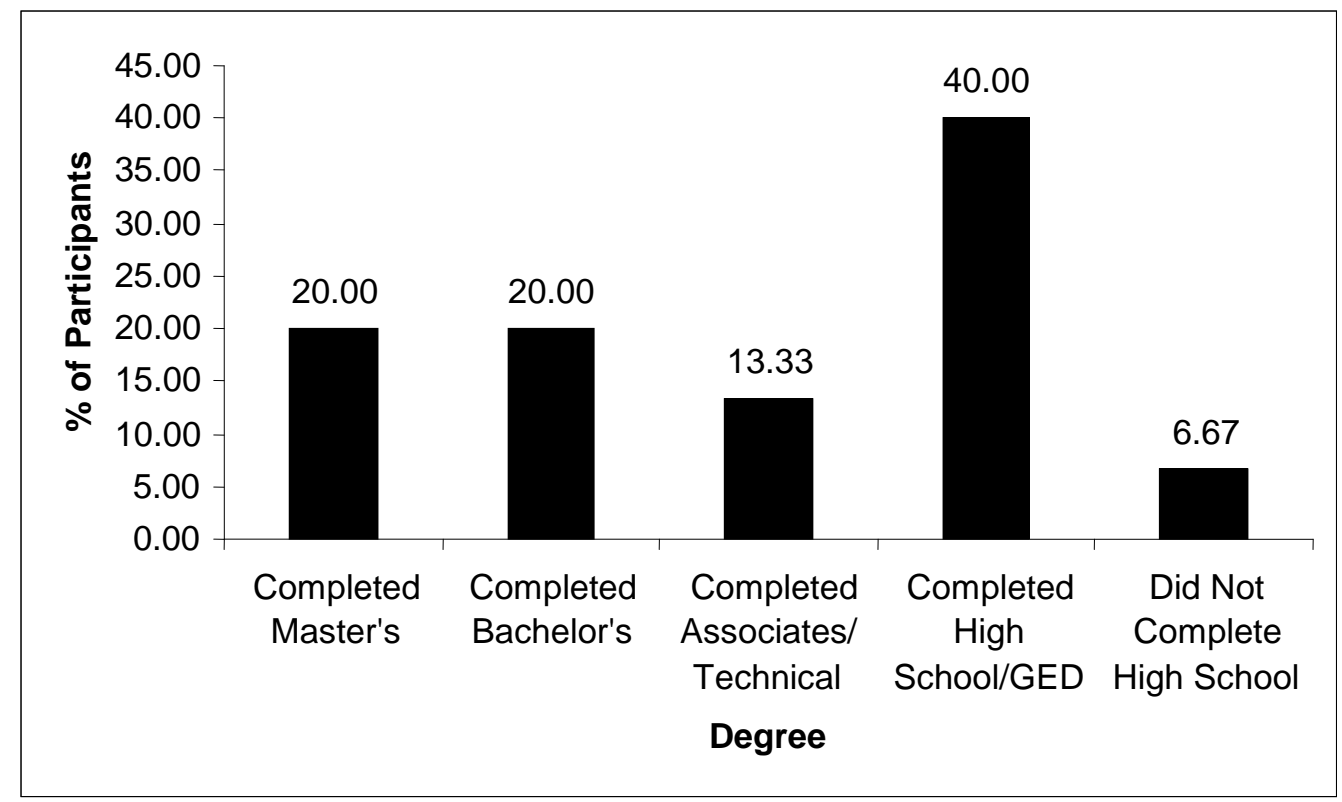

Figure 3. Highest educational degree obtained by participants' fathers.

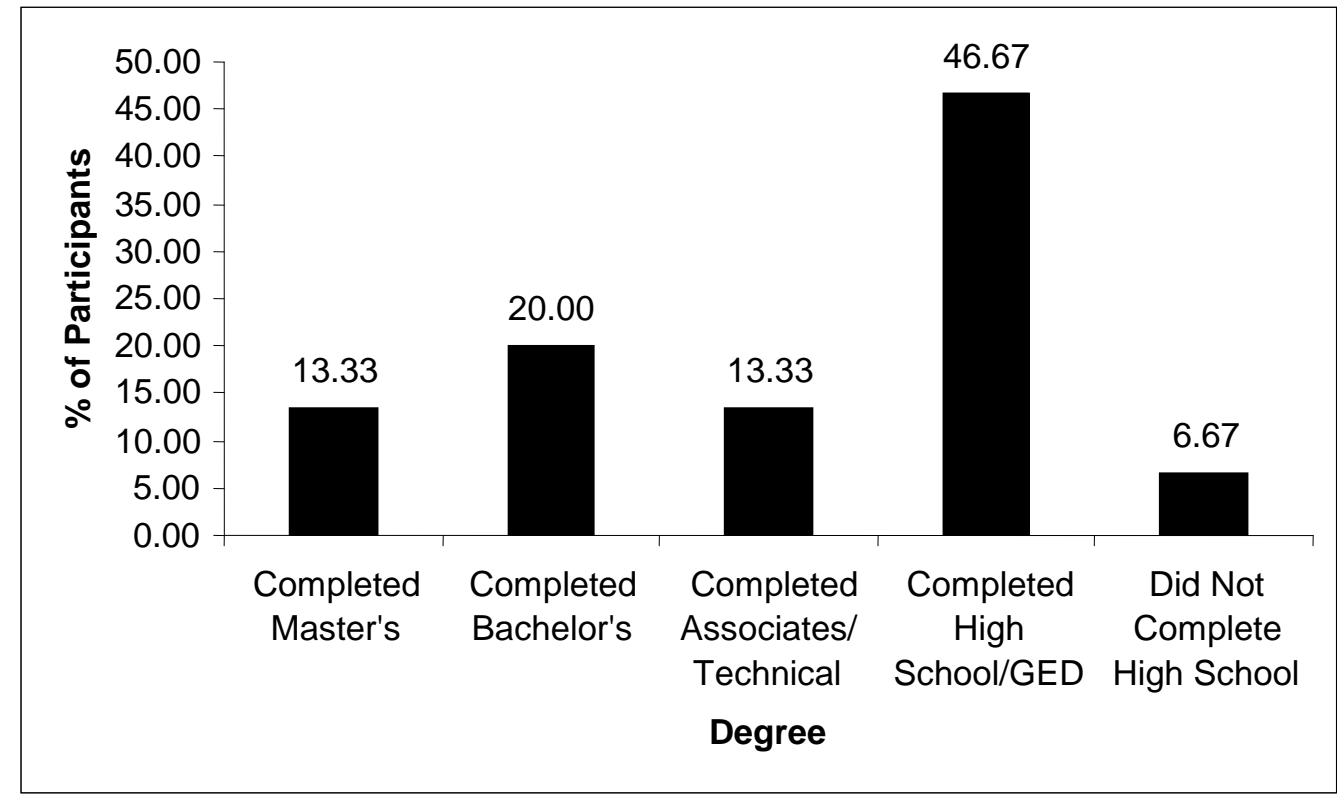

Figure 4. Highest educational degree obtained by participants' mothers. 


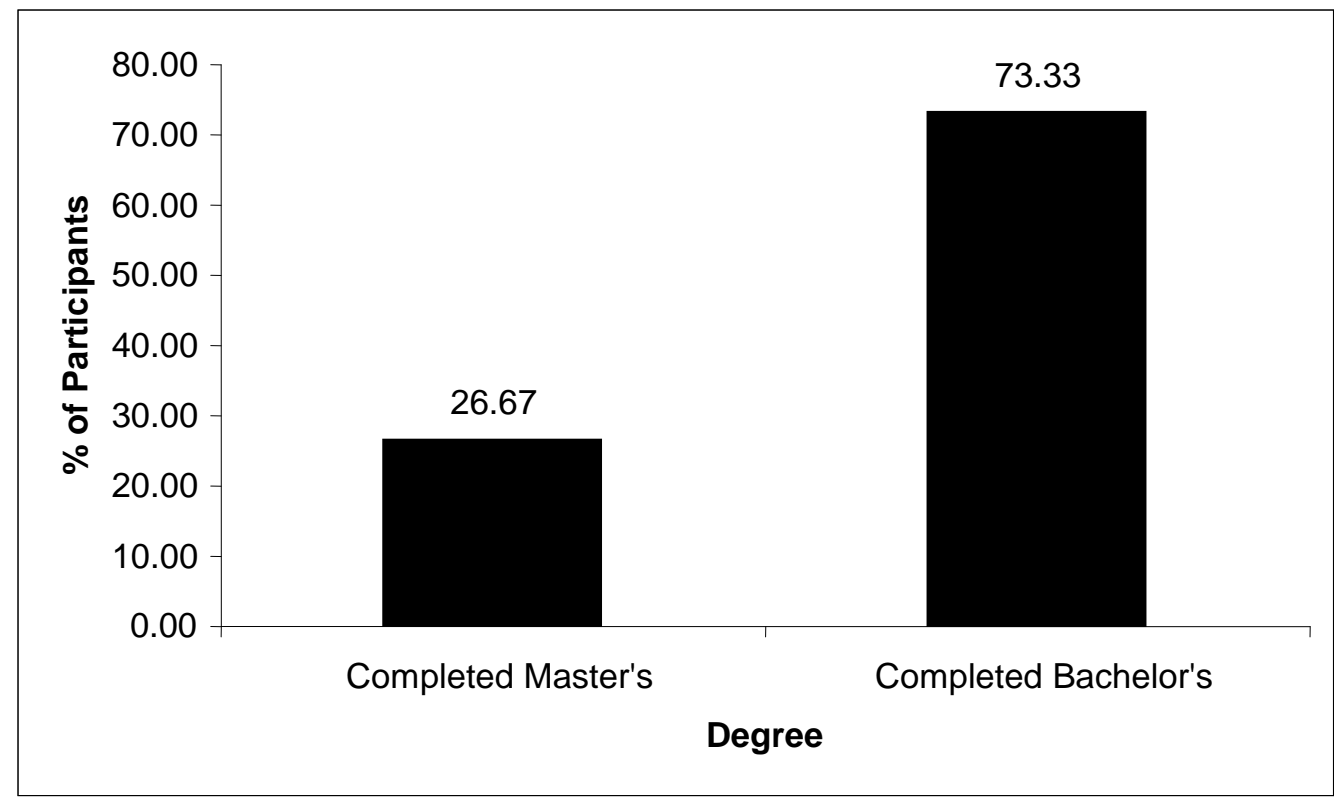

Figure 5. Highest educational degree obtained by participants.

As children, the majority of the participants were of middle socioeconomic status.

By adulthood the participants were of mostly upper middle socioeconomic status (see Figures 6 and 7).

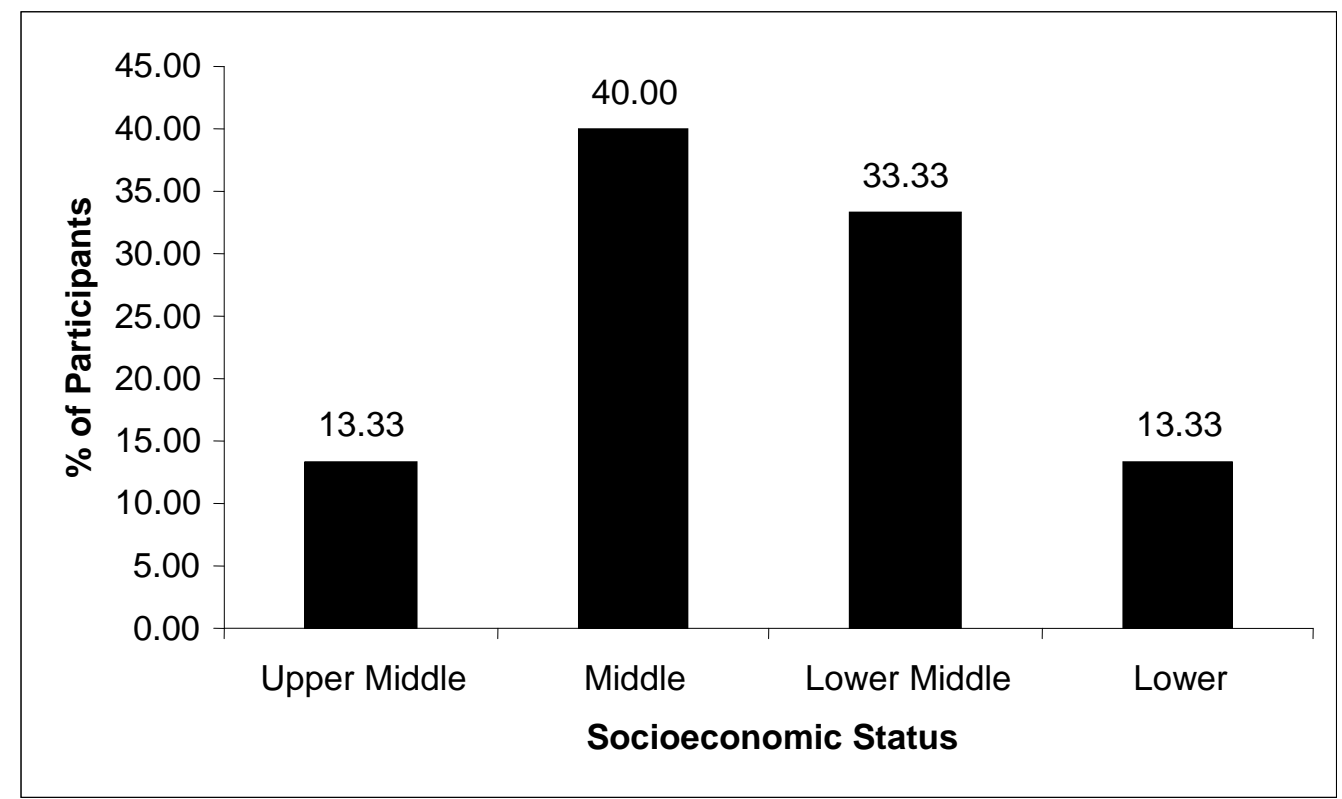

Figure 6. Participants' socioeconomic status as children. 


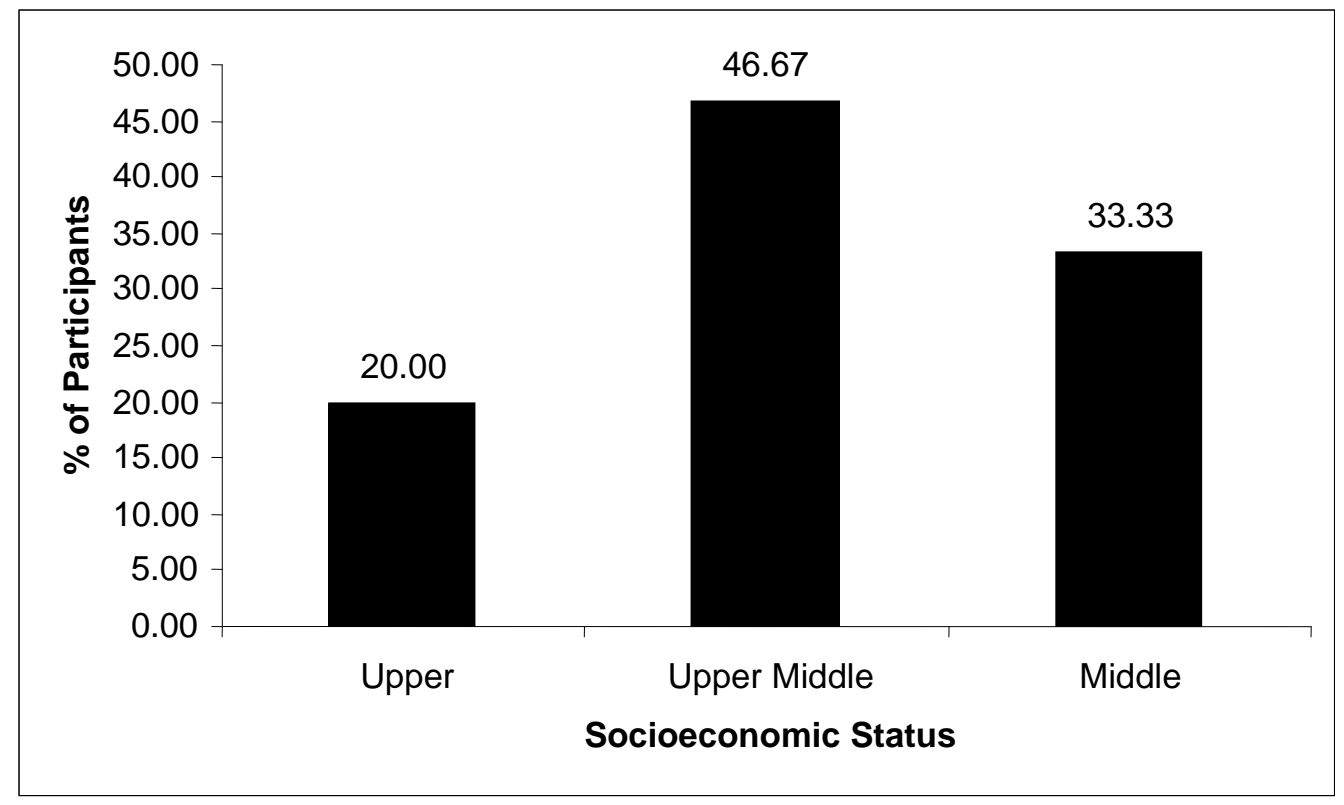

Figure 7. Participants' socioeconomic status as adults.

Participants' education and training related to English language learners is summarized in Table 3. A slight majority of the participants had no specializations or majors related to educating students learning English. Approximately half of the participants had at least one endorsement related to educating English language learners, and approximately half of the participants had done some personal study of sheltered instruction. A slight majority of the participants completed 55 to 109 hours of sheltered instruction training (see Figure 8). 
Table 3

Participants' Education and Training Related to Teaching English Language Learners

Variable

Frequency

Percent

Specializations and majors for all of participants' degrees

Not related to English language learners

At least one related to educating

English language learners

Completed endorsements of participants

Not related to English Language

Learners

At least one related to educating

English language learners

Personal study of sheltered techniques

None

At least some

10

66.7

5

33.3

7

46.7

8

53.3

8

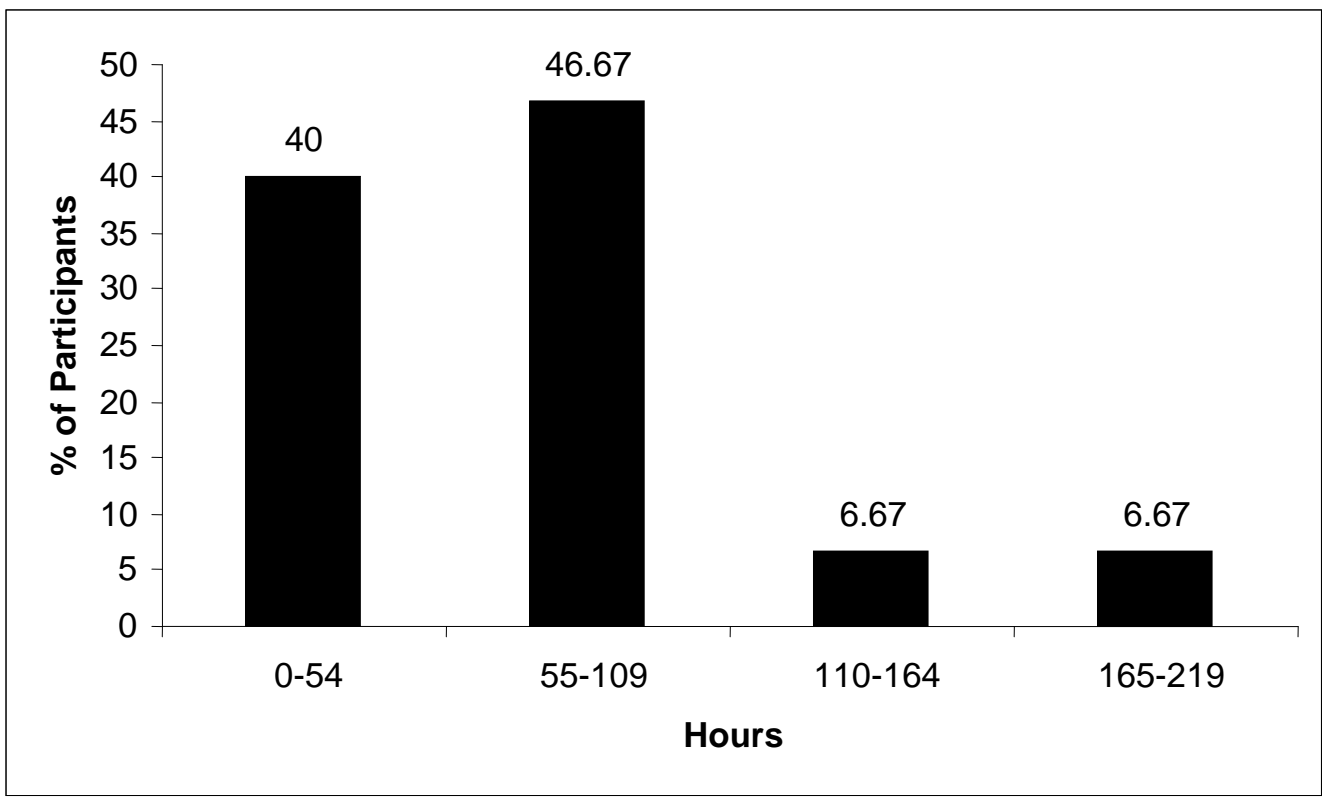

Figure 8. Total hours of participants' sheltered instructional training. 
The majority of the study's participants have 10 years or less of teaching experience in middle schools and the same amount of experience teaching at least one English language learner (see Figures 9 and 10).

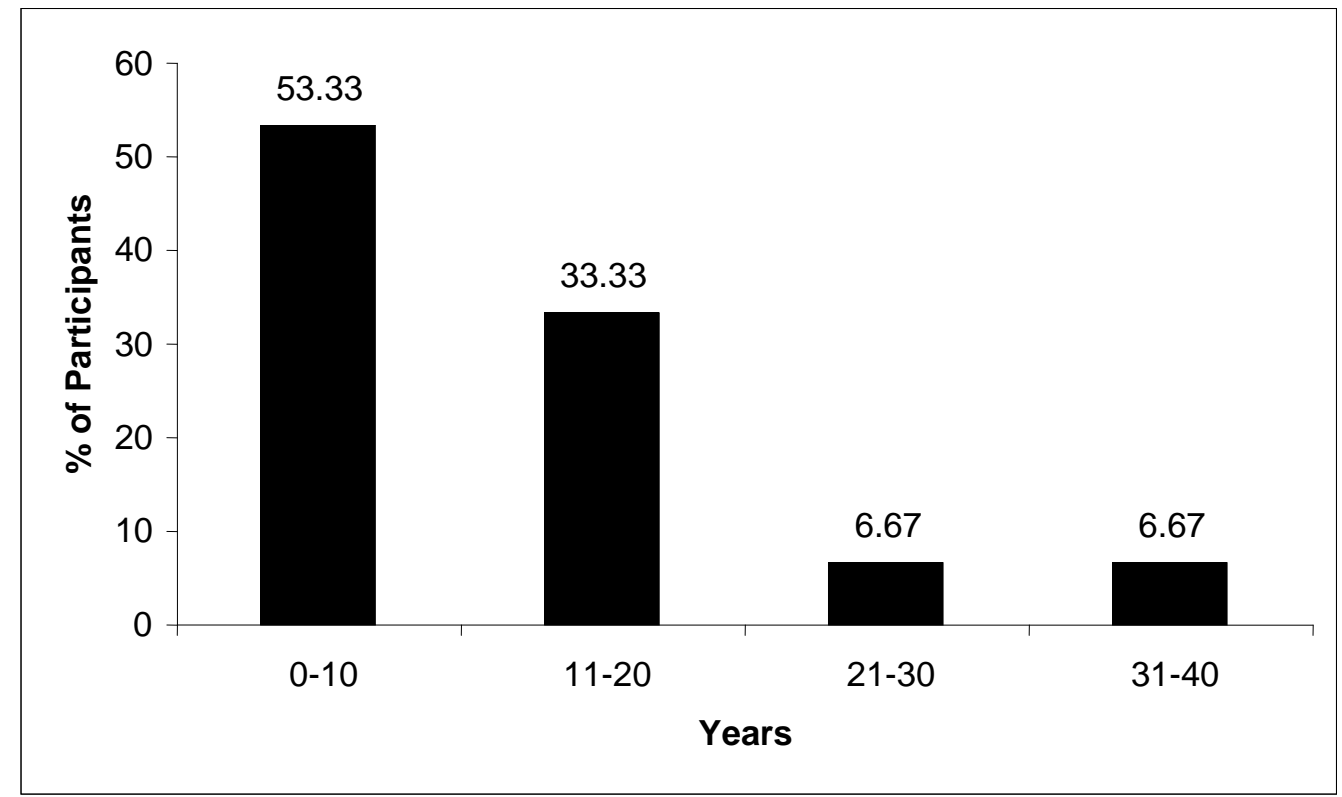

Figure 9. Total years of participants' middle school teaching experience.

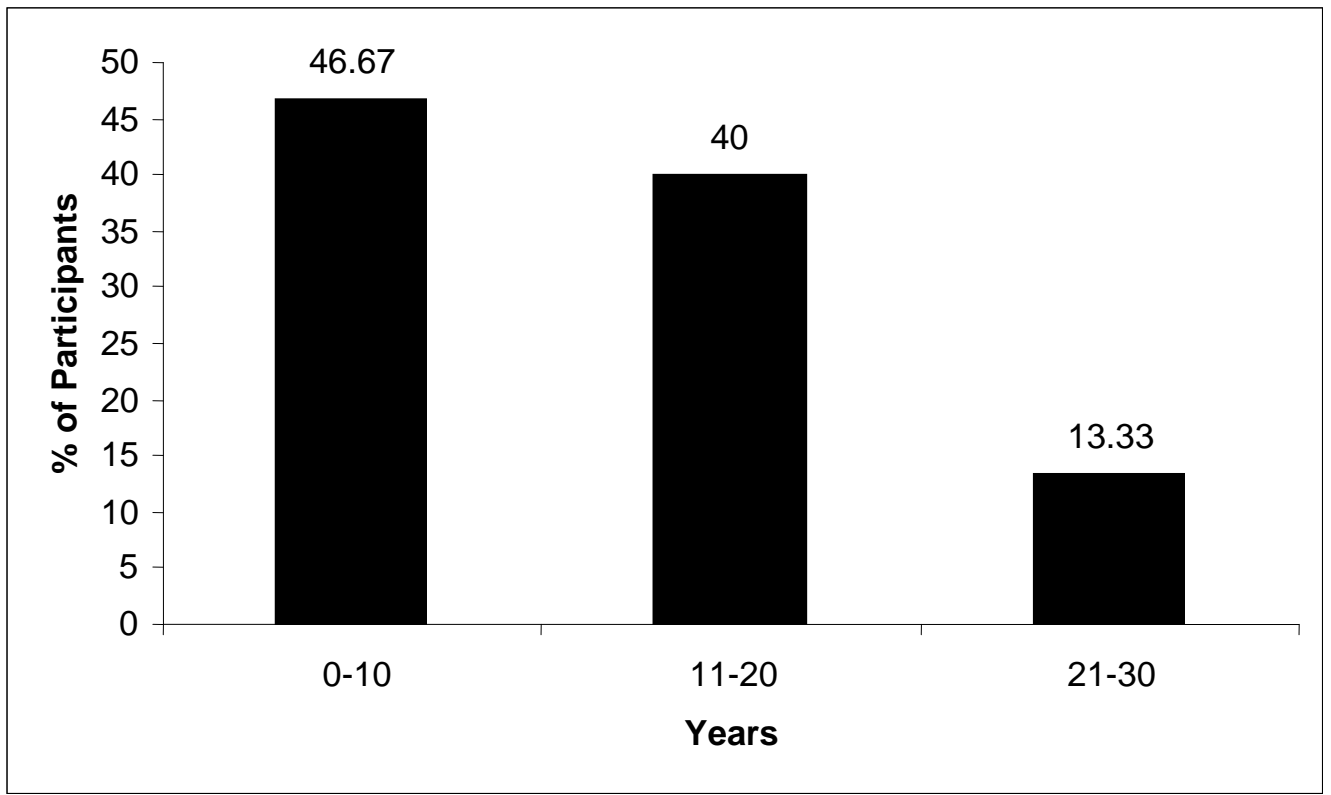

Figure 10. Total years of participants' experience teaching at least one English language learner. 
After the data collection and analysis of the quantitative data were complete, Phase 2 of the study began. Six participants were selected for the semi-structured interviews (i.e., the three highest SIOP scorers and three lowest SIOP scorers). Results from the data analysis of the quantitative and qualitative data informed the selection process of interviewees. Participants that represented both typical and outlier cases were selected for interviewing to reach a deeper understanding of what may have caused some participants to score higher on the SIOP than others. For example, the CIQ results indicated some participants understood the role of multicultural education in student learning, while others did not. Participants who did not seem to understand the connection between student learning and multicultural education, yet were the highest SIOP scorers, were selected for interviewing, as were those who did seem to understand the student learning-multicultural education connection, but were the lowest SIOP scorers.

\section{Instrumentation}

Several instruments were used to measure participants' attitudes and levels of implementation of sheltered instructional techniques. Important considerations were reliability and validity. Factors that can threaten an instrument's reliability and result in unreliable data include: (a) ambiguous or unclear questions on an instrument; (b) nonstandardized test administration; and (c) participants feeling fatigued or nervous, misinterpreting directions or questions, or guessing on tests (McMillan \& Schumacher, 1997; Rudner, 1994).

To address these factors, I standardized the administration of each instrument by providing each participant with the same written and oral directions that clearly explained 
the instructions, placed the participant at ease, and encouraged each individual to ask for clarification as needed. My intention was to avoid administering the instruments to participants at the end of their workday or week, the times they were most likely to be fatigued. However, for some participants these were the most convenient or least stressful times to complete the instruments because they did not have to think about preparing for or teaching a class.

An instrument's validity or the extent to which the instrument measures what it intends to measure (Salkind, 2004), may be threatened by several factors. Invalid scores may result from poorly designed questions, measures of variables, or the inability to make useful predictions from scores (Creswell, 2008). Detailed information regarding the validity of each instrument is reported in the following paragraphs.

Demographic Questionnaire. During Phase 1 of the study, demographic data were collected from the participants. The Demographic Questionnaire asked participants to respond to questions organized into three categories: (a) personal information, (b) teaching experience and training, and (c) international travel and foreign language use (see Appendix A). The personal information section gathered data about participants' gender, age range, and socioeconomic status. Information about participants' completed degrees and endorsements, the extent of their sheltered training and study, and experience teaching middle school and English language learners was collected in the teaching experience and training section. Information on any international travel and intermediate to fluent proficiency in a non-English language was gathered in the travel and foreign language use section. The Demographic Questionnaire was based on the personal information items of the MES. One item, regarding participants' sexual orientation, was 
eliminated because of my existing relationship with the participants. Some individuals may believe their sexual orientation is a private matter and do not want to share this information. I was also concerned that participants would choose not to participate in the study due to (a) concerns that the information about sexual orientation might be divulged after the study, or (b) feelings of discomfort about working with a colleague who knew their sexual orientation. Further, I believed the other variables on the instrument provided sufficient information to test the relationship with sheltered instruction. Additional items, related to independent variables that may affect the extent participants use sheltered instructional techniques were added (i.e., sheltered instruction training and study, number of years teaching middle school, number of years teaching English language learners, travel outside the U.S., and languages other than English spoken at the intermediate level or above).

I received no specialized training to administer the Demographic Questionnaire. However, I relied on my experience administering other assessments and attempted to maintain neutral body language and speech during the administration (e.g., when a participant asked a question) and a relaxed atmosphere (e.g., not looking at the clock or preparing to go home), so the participant did not feel rushed.

Multicultural Efficacy Scale. Phase 1 continued with the 35-item MES that measured participants' beliefs about multiculturalism in three areas: (a) experience with others different from themselves, (b) opinions about multicultural educational practices, and (c) a self-assessment of their ability to incorporate multicultural practices into classroom instruction (Guyton \& Wesche, 2005) (see Appendix B). 
Consistent, stable scores across repeated administrations of an instrument contribute to its reliability (Creswell, 2008). Likewise, individuals who answer questions in a particular way should answer similar questions in a consistent manner. To assess the internal consistency of the respondents' answers on the MES, Guyton and Wesche (2005) used Cronbach's alpha and computed a score of .89 for the 35-item scale and 0.72 to 0.93 for the subscale alphas of experience with diversity, attitude about diversity, and selfassessment of teaching efficacy related to diversity (Guyton \& Wesche, 2005). I calculated the internal consistency of the answers of my study's participants and found Cronbach's alpha to be 0.87 for the 35 -item scale, 0.87 for experience with diversity, 0.65 for attitude about diversity, and 0.94 for self-assessment of teaching efficacy related to diversity. These results show participants in my study answered the questions on the subscales of the MES in a consistent manner.

To improve the clarity and precision of the instrument's questions, Guyton and Wesche (2005) conducted an analysis of participants' responses and eliminated questions that did not accurately measure the concepts they intended to measure. I conducted a similar analysis with the CIQ by administering the questionnaire to educators who would not participate in the study. Based on the analysis, the questions were revised to narrow their foci and list examples of key terminology.

The content validity, or extent to which an instrument's questions are representative of all possible questions that could be asked about the content or skills (Creswell, 2008), was reviewed for the MES. Guyton and Wesche (2005) sent the instrument to more than a dozen experts in the field of multicultural education to request their feedback. Based on the experts' recommendations regarding the instrument's scale 
and questions, the authors revised the MES (e.g., some sections were deleted) (Guyton \& Wesche, 2005).

Another issue of validity (i.e., inaccurate predictions from scores) is examined by criterion-related validity. This type of validity determines whether an instrument's scores do in fact predict the outcome that they are expected to predict (Creswell, 2008). To improve the extent to which the MES predicted a teacher's effectiveness in multicultural settings, Guyton and Wesche (2005) conducted two stages of statistical analyses before finalizing the item selection for the instrument. Questions that did not effectively measure the specific area of interest related to multicultural education were eliminated (Guyton \& Wesche, 2005). They also conducted a confirmatory factor analysis and reliability analysis on the MES. Factor loadings under 0.50 were suppressed. Results demonstrated that all 20 of the efficacy items loaded on Factor 1, eight of the attitude items and seven of the attitude items loaded together. Because the general knowledge and instructional knowledge items did not seem to form identifiable factors, the two knowledge sections were eliminated from the final instrument. Based on these results, Guyton and Wesche (2005) asserted the instrument is useful for diagnosing teachers' levels of multicultural efficacy and is an indicator for the type of professional development needed. They also granted permission for the use of the Multicultural Efficacy Scale in my study (see Appendix C).

I did not have any specialized training to administer the MES. When administering the MES, I relied on previous experience administering assessments by using neutral body language and speech during the administration and maintaining a relaxed atmosphere. While reading the instructions aloud to each participant, I stressed 
the phrase in the directions that referred to there being no correct answers, indicating my interest in people's opinions.

Sheltered Instruction Observation Protocol. Phase 1 concluded with the use of the SIOP as a behavioral instrument. The SIOP was used to measure participants' usage of sheltered instructional techniques in the following areas of instruction: (a) preparation, (b) building background, (c) comprehensible input, (d) strategies, (e) interaction, (f) practice or application, (g) lesson delivery, and (h) review and assessment (Echevarría et al., 2008) (see Appendix D). Each participant's class was observed and scored twice with the SIOP. Each observation was videotaped.

To establish the internal consistency of the SIOP, Guarino and associates (2001) calculated the Cronbach's alpha for each of the instrument's subscales. Four teachers experienced with sheltered instruction analyzed videos of classroom instruction and scored the teachers using the SIOP. Specialists had classified three of the videotaped lessons as highly representative of sheltered instruction, whereas the other three were not. The observational protocol was divided into three subscales; Cronbach's alpha was calculated for each subscale. Results ranged from 0.91 to 0.95 demonstrating that the SIOP was a reliable instrument for distinguishing between educators who used sheltered techniques and those who did not. The Cronbach's alpha calculations for the two classroom observations I conducted were 0.85 for the first observation and 0.83 for the second observation, indicating the SIOP was a reliable indicator of the extent participants used sheltered techniques in their classroom instruction.

To address bias that I might have brought to scoring the use of sheltered techniques in classroom instruction, interrater reliability was calculated for $10 \%$ of the 
observations. With one other individual, I scored classroom instruction for its use of sheltered techniques with the SIOP. This individual had experience teaching with sheltered instructional techniques and using the SIOP for observations. Moreover, this individual was not an administrator, so participants would not be concerned that information obtained during the observations would be used in performance evaluations. Two observations were conducted jointly at the beginning of scoring all of the observations and one observation was scored jointly toward the end of scoring the observations. Scores from each joint observation were compared and discrepancies were discussed. On most items an exact agreement was reached, on a few items the difference was as much as three points. According to the Pearson Product-moment Correlation, the rate of correlation across 24 items was statistically significant $(r=.92 ; p<.01)$.

Guarino and associates (2001) examined the criterion validity of the Sheltered Instruction Observation Protocol using direct discriminant formula analysis. This analysis was conducted on the three subscales of the instrument to predict membership in one of two groups: participants who used sheltered techniques and those who did not. At $\alpha<.001$, no univariate or multivariate within-cell outliers were found. The Wilks' lambda was calculated to be 0.117 ; results showed instructional types explained $94 \%$ of the function variability. Univariate tests indicated all three predictors were statistically significant for distinguishing between educators who used sheltered techniques and those who did not. The stability of the classifications was checked with cross-validation resulting in a $95.25 \%$ correct classification rate.

Echevarría and colleagues (2008) granted permission for use of the SIOP for this study (see Appendix E). My experience teaching and planning sheltered lessons and 
knowledge of second language acquisition provided the necessary background to understand the components of the sheltered instruction model. Prior to this study, I had used the SIOP to conduct classroom observations. Additionally, I reviewed the rubrics and compact disc provided in Making Content Comprehensible for English Learners: The SIOP Model materials in preparation for scoring classroom instruction.

Classroom Instruction Questionnaire. Phase 2 of the study, the collection of qualitative data, began with the CIQ. The questionnaire, consisting of six open- and close-ended questions, is designed to learn about each participant's (a) use of multicultural educational practices in the classroom, (b) the participant's attitude about educating diverse students, and (c) the participant's perception of his or her classroom use of sheltered instructional techniques (see Appendix F). Because the CIQ consists of open- and close-ended questions, the advantages of both types of questions are incorporated into the instrument. Close-ended questions are useful in supporting theories and concepts in the literature (Creswell, 2002) and are practical because respondents answer the questions using the options provided. This allows for comparing and coding responses for data analysis (Creswell, 2008). Conversely, open-ended questions are more difficult and time consuming to code and analyze because responses must be categorized by theme and subsequently counted. Benefits of using open-ended questions include allowing participants to explain personal experiences in detail and share those experiences outside the scope of the close-ended questions (Creswell, 2002). I developed the CIQ with assistance from my advisor, who has experience using interviews and surveys in research. 
Although both the MES and CIQ measure participants' attitudes about multicultural educational practices, the CIQ is specific to the participants' classroom practices related to multicultural education. While responses on the MES are measured on a five-point scale, the majority of the questions on the CIQ are open-ended questions, which allows the participants to tailor their answers to their specific teaching context. To improve the questionnaire's reliability, I standardized the instrument's administration by using clearly written and explained instructions, placing participants at ease with my tone of voice and body language, encouraging participants to ask clarifying questions as needed, and avoiding the administration of the questionnaire to participants during times they were likely to be fatigued or distracted.

To address its content validity, the CIQ was reviewed by experts in multicultural education and the education of English language learners, district instructional coaches, and classroom teachers who were not part of the study. I solicited these individuals' feedback regarding the extent the questionnaire was representative of all the possible questions that could be asked to understand participants' attitudes about multicultural education, teaching diverse students, and the extent teachers say they are using sheltered instruction. In response to the feedback, I revised the questions to (a) narrow their focus, (b) ask only one question per item, and (c) provide examples of key terminology used in the questions. I received no special training to administer this questionnaire and relied on previous experience administering other similar assessments.

Semi-structured Interviews. Phase 2 of the data collection concluded with interviewing six participants using the semi-structured interview questions (see Appendix G). The interview questions, consisting of three open-ended questions, were used to 
better understand participants' attitudes about multicultural education, teaching diverse students, and using sheltered instructional practices. The interviews were audio taped. I began each interview by explaining the purpose (i.e., to further my understanding about the relationship between multiculturalism and teachers' use of sheltered instructional techniques in the classroom). Moreover, I explained to each participant that the interview would be recorded and later transcribed. I also asked each participant if there were any questions before starting the interview. During the interviews, I asked participants the three original semi-structured interview questions, as well as questions I had developed after analyzing participants' written responses from the CIQ. To understand participants' answers to these questions and their thoughts about what might explain teachers' implementation of sheltered instructional techniques and the lack thereof, I used various types of questions (e.g., follow-up, probing, specifying, indirect, and interpreting).

Reliability for the semi-structured interviews was addressed in a manner similar to that used for the CIQ (i.e., providing clear instructions, placing participants at ease, encouraging participants to ask for clarification as needed, and avoiding interviewing at times participants were likely to be fatigued or distracted). Prior to the interviews, I studied Kvale and Brinkman's (2009) indicators of a quality interview: (a) the degree of spontaneous, detailed, specific, and relevant answers from the interviewee; (b) the amount of short interviewer questions and longer interviewee answers; (c) the extent to which the interviewer follows up and clarifies the meanings of the relevant aspects of the answers; (d) to a great degree, the interview being interpreted throughout the interview; (e) the interviewer trying to verify his or her interpretations of the subject's answers over the course of the interview; and (f) the interview being self-reported, a self-reliant story 
that does not require much additional explanation. I used these criteria as well as other resources in the book as a guide for conducting the interviews. I also developed a threepage summary that I could refer to as I conducted the interviews, which included a protocol for conducting the beginning, middle, and end of the interview; the criteria for an interview; types of interview questions; and Ellen Mayo's method of interviewing. To become familiar with the interview equipment and procedures, I practiced interviewing someone who was not part of the study.

Issues of validity specific to qualitative data. Maxwell (2005) concluded there were distinctions between quantitative and qualitative designs related to how they addressed threats to validity. According to Maxwell, qualitative proposals should emphasize how the researcher will rule out specific, plausible alternatives and threats to the researcher's interpretations and explanations. Quantitative researchers generally attempt to design controls in advance to manage anticipated and unanticipated threats to validity (e.g., framing explicit hypotheses in advance of collecting the data and using tests of statistical significance). However, qualitative researchers often do not have the benefit of using tools that rule out or control plausible threats (e.g., previously planned sampling strategies and statistical calculations that control for threats). Instead, researchers involved with qualitative designs must attempt to exclude most validity threats after the research has begun (Maxwell, 2005).

Two broad threats to validity, researcher bias and reactivity, are often discussed in relationship to qualitative studies (Maxwell, 2005). Researcher bias occurs when data are selected to fit the researcher's existing theory, or data are selected because they stand out to the researcher (Miles \& Huberman, 1994). In the case of this study, possible biases 
include (a) I believe teachers' attitudes about multiculturalism and subsequently diverse students may influence the extent they use sheltered instructional techniques in their classrooms, (b) I believe culturally responsive teaching is an instructional method that is essential for the success of students from diverse backgrounds, and (c) I believe sheltered instruction is an essential component of content construction for English language learners.

The ways I attempted to address researcher bias included avoiding leading questions in the interviews and explaining to participants the importance of their particular thoughts and opinions to this study. Second, I looked for evidence that could challenge my conclusions (Maxwell, 2005). The first participant I interviewed discussed the role self-reflection played in learning how to use sheltered instructional techniques. From her perspective, being self-reflective substantially influenced her implementation of sheltered instruction in her classroom teaching. To ascertain if the use of self-reflection was a factor in the other participants' use of sheltered instruction, I asked each of them what role, if any, self-reflection played in their teaching. Third, verbatim transcriptions of interviews and video recordings of classroom instruction provided me with a rich and significant amount of data, which I could mine for evidence that challenged my conclusions. Finally, respondent validation was another method I used to identify my own biases and misunderstandings of what I had observed. In respondent validation, the researcher systematically asks for feedback about the data and his or her conclusions from the individuals being studied. The purpose of this technique is to exclude the possibility of misinterpreting participants' statements or actions and the perspectives they have about what is occurring (Maxwell, 2005). I used this technique during the 
interviews and afterwards. During the interviews, I used follow-up and clarification questions to confirm my understanding of the ideas put forth by the interviewees. For example, when I asked one of the participants about the role of self-reflection in her teaching, she initially stated self-reflection was more effective when the teacher had a smaller group of students. By using clarification questions, I learned that the participant wanted to reflect by conversing about instructional practice with other teachers instead of reflecting alone. When I quoted the participants, I shared the quote and its context with the participants to check that I had accurately understood them. Each participant confirmed that I had. One participant asked that I complete a quote by emphasizing what she had said about students in the interview. I followed her suggestion.

Reactivity refers to the influence the researcher has on a setting or the individuals being studied (Maxwell, 2005). Because I have worked at the study site for 10 years in some capacity related to the education of English language learners, participants know I support the use of sheltered instruction in content classrooms and I advocate for English language learners related to program design, scheduling, instruction, and professional development. Participants could act or make statements that are not necessarily accurate depictions of what they believe or do in the classroom. Instead, they could behave in a manner consistent with what they perceive I believe or am looking for in the study. Conversely, my years of experience working at the research site served as an asset because I could compare participants' responses and behavior during the study with patterns of behavior observed before the study. During data collection, I found participants to be honest in describing their perspectives on multicultural education, teaching diverse students, and using sheltered instruction in their classrooms. 
Participants disclosed areas of their practice they believed needed improvement (e.g., "But, it's [multicultural education practices] one of those slippery concepts for me that I want to see more of what it is...looks like to make sure that I'm doing it right."), times they believed they had made mistakes (e.g., "I remember one of the...first interactions I had with him [indigenous Latino boy]...it was a discipline issue. And he didn't look at me, and I approached that from my background, which is...you look at people when they're talking to them [you]...I read the way his body language was as shame...so he wouldn't look at me...I didn't understand that that was part of his culture that you don't look authority figures or adults in the eye..."), and how deeply working within a school system dominated by mainstream culture affects them (e.g., "I think the biggest challenge [related to educating diverse students] is to be immersed in a system that...doesn't understand the needs to serve multicultural students... when things are presented, my reaction is totally different than the rest [of my team members], but because I am one, many times I have to think if I want...[to] open my mouth. It's going to create conflict and then I'm going to be labeled...instead of me being understand [understood] as [a] representative voice of the students...because if I feel it this way and then the same thing is going to be introduced to the students, how are the students going to feel?"

\section{Data Collection}

Before the data collection and analysis began, the significance level for the data analysis was set to $p<.05$. As a result, there was a five percent chance of rejecting the null hypothesis (i.e., there is no relationship between teachers' attitudes about multiculturalism and their use of sheltered techniques, when the null hypothesis was in fact true). Conversely, there was a five percent chance of accepting the null hypothesis 
when it was actually false (Salkind, 2004). I collected data with the Demographic Questionnaire and several quantitative measures: the MES, SIOP, and question six of the CIQ; and two qualitative measures: the majority of the CIQ and semi-structured interviews.

The data collection began with the 14-item Demographic Questionnaire designed to collect information about participants' background. The MES, a Likert-scale survey of 35 items gathered participants' attitudes about multiculturalism. Two observations were conducted in each participant's classroom. Each observation was videotaped and scored with the SIOP. The videotapes were used to initially score lessons as well as review given scores. The CIQ consisted of six open and closed-ended questions designed to measure participants' attitudes about multicultural education, teaching diverse students, and using sheltered instructional techniques in their classrooms. The last form of data collection involved the semi-structured interviews that consisted of three initial questions and follow-up questions designed to understand the participants' thinking on a deeper level, as well as possible explanations for the high SIOP scores. The interviews were approximately 45 minutes in length. To allow subsequent transcription, the interviews were audio taped.

Measuring variables. Five different types of variables are characteristic of quantitative research: (a) dependent, (b) independent, (c) control, (d) intervening, and (e) confounding. A dependent variable is the outcome the researcher is attempting to explain (Creswell, 2008). In this study, each participant's total score on the SIOP instrument is a dependent variable. Independent variables are the factors that may influence the outcome (Creswell, 2008). The responses on the Multicultural Efficacy Scale are examples of 
independent variables. Control variables (e.g., gender and age on the Demographic Questionnaire), are another type of independent variable, used as possible explanations for the outcome (Creswell, 2008). In this study, items such as gender and age on the Demographic Questionnaire are control variables (see Appendix H for a Codebook of the Quantitative Data).

Intervening variables mediate the effects of the independent variable on the dependent variable (Creswell, 2008). The intervening variable exercises an influence on the dependent variable that is distinct from the independent variable. If the researcher does not identify an intervening variable that is influencing the dependent variable, he or she may overestimate the independent variable's influence on the dependent variable or falsely attribute a relationship between the independent and dependent variables. To identify any intervening variable in this study, multiple control variables were measured. Second, both the CIQ and semi-structured interviews included open-ended questions. The intervening variable of self-reflection was identified via the interviews.

Though confounding variables may influence the relationship between the independent and dependent variable, confounding variables cannot be directly measured (Creswell, 2008). This is because the effects of confounding variables cannot be easily separated from those of other variables. Therefore, it is important to explain limitations on the results. For example, if the other independent variables in the study had been controlled and a significant relationship was found between non-Caucasian, nonEuropean participants and high scores on the Sheltered Instruction Observation Protocol, multiple factors related to race could provide a plausible explanation for this relationship. In addition to their diverse racial and ethnic backgrounds, this group of participants might 
have grown up in multiracial or multiethnic families and communities where they were exposed to different ways of thinking and being. Because of their experiences living and interacting with people of diverse cultures, respondents in this group may tend to pay close attention to activating students background knowledge and teaching students how to use strategies to complete tasks and solve problems. Both instructional approaches are part of the sheltered instruction technique. For further information about the variables measured on the quantitative instruments (i.e., type of scale with which the variables are measured and how the variables are scored) see Appendix $\mathrm{H}$, which includes variable information exclusively on the quantitative measures.

Rather than investigate a cause and effect relationship between dependent and independent variables, as is done with quantitative research, qualitative research is designed to explore and understand a single phenomenon (Creswell, 2008). For this study, qualitative methods were used to arrive at an understanding of the dependent variable, while quantitative methods were used to examine the relationship between the dependent and independent variables.

\section{Data Analysis}

This study involved the analysis of both quantitative and qualitative data. Analysis of the quantitative data included the use of descriptive and inferential statistics, while analysis of the qualitative data required exploring and coding the data to develop themes (Creswell, 2008). The process used to analyze the quantitative and qualitative data are illustrated in Figure 11. 


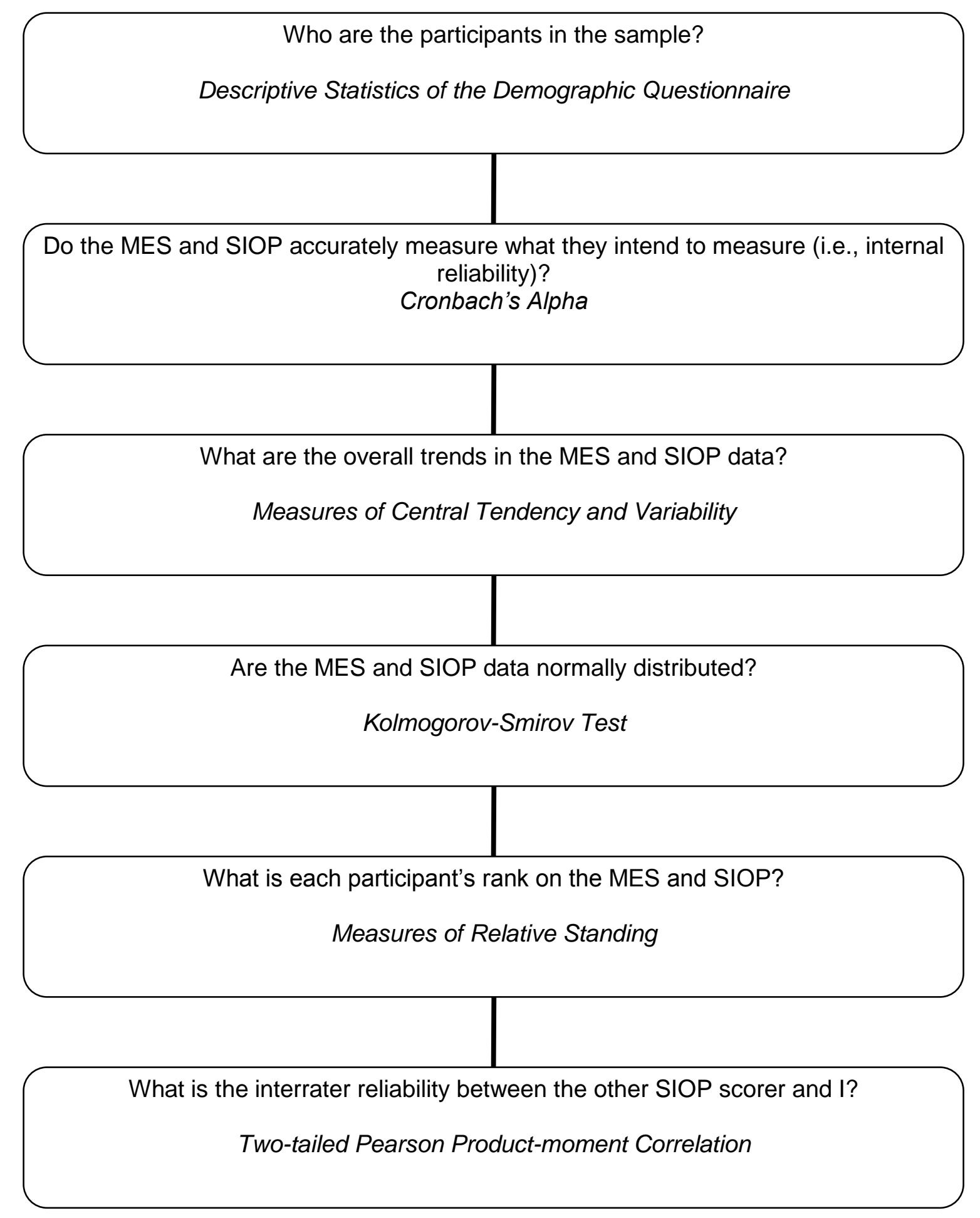

Figure 11. Flowchart of quantitative and qualitative data analysis. 
Is there a significant relationship between teachers' attitudes about multiculturalism and their level of implementation of sheltered techniques in the classroom?

Two-tailed Pearson Product-moment Correlation

Is there a significant relationship between variables on the Demographic Questionnaire and the total SIOP score?

Two-tailed Pearson Product-moment Correlation

How do participants rate themselves on their use of sheltered instructional techniques?

Descriptive Statistics of the Quantitative Section of the CIQ

What are the overall trends in the quantitative CIQ data?

Measures of Central Tendency and Variability

Is the quantitative section of the CIQ normally distributed?

Kolmogorov-Smirov Test

What is each participant's rank on the quantitative section of the CIQ?

Measures of Relative Standing

Is there a significant relationship between participants' total SIOP score and their selfevaluation of their use of sheltered instructional techniques?

Two-tailed Pearson Product-moment Correlation

Figure 11. Flowchart of quantitative and qualitative data analysis continued. 


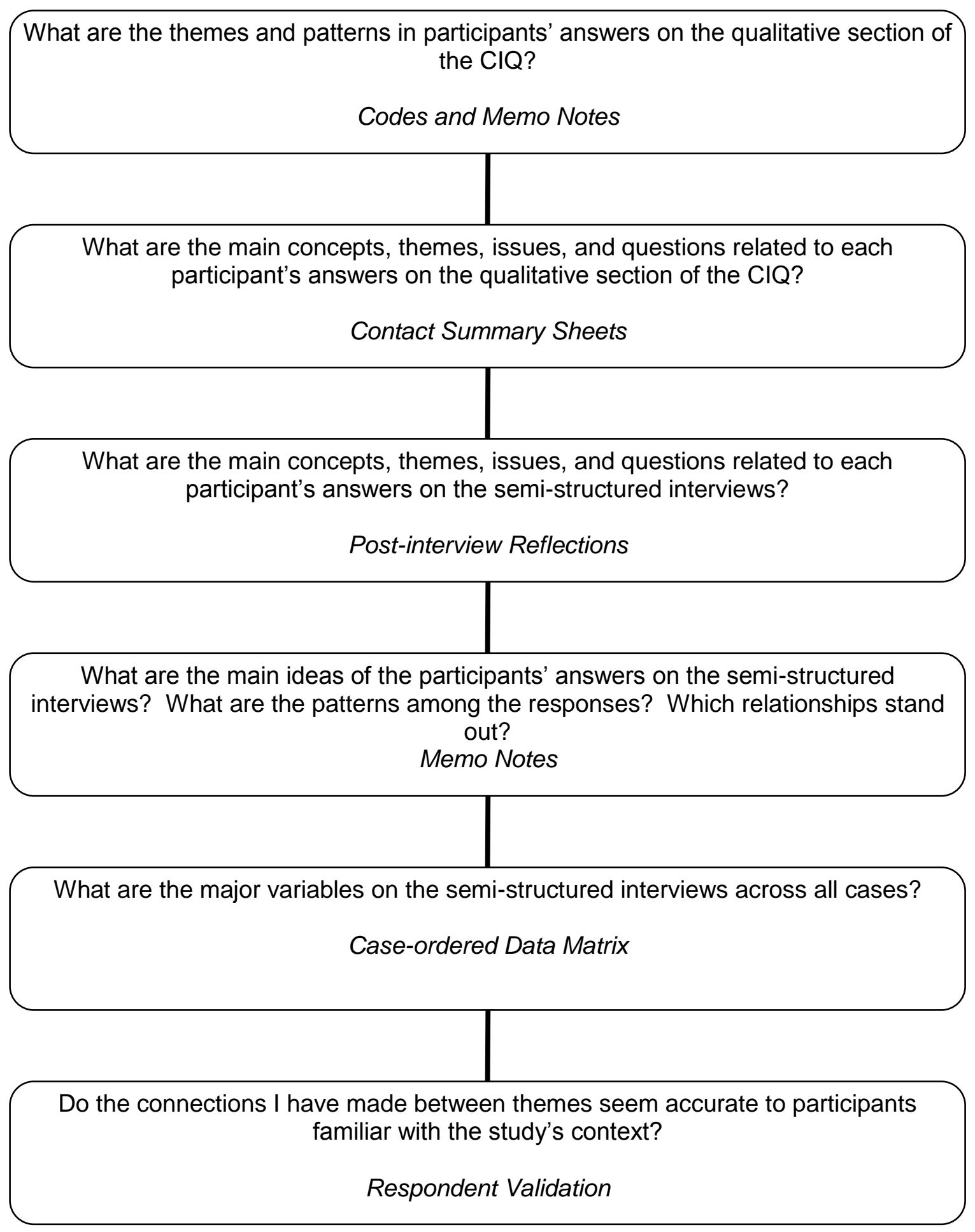

Figure 11. Flowchart of quantitative and qualitative data analysis continued. 
Quantitative. The quantitative data analysis began with the use of descriptive statistics to describe the sample's general characteristics according to the Demographic Questionnaire. Next, Cronbach's alpha was calculated for the MES and SIOP to compare internal reliability results from this study with those of the instruments' authors. A descriptive analysis was conducted on the MES and SIOP to determine the overall trend and distribution of data, illustrate the extent to which scores vary, and highlight how a particular score compares to others (Creswell, 2008). Calculating the central tendency of the data identified the mean or average score of all participants, the median or result of a typical participant, and the mode — the most frequently occurring score (Creswell, 2008). Measures of variability (i.e., range, variance, standard deviation, skewness, and kurtosis) were examined to determine the extent to which the scores varied from one another. Additionally, the Kolmogorov-Smirov test was used to check the significance of any deviations from the norm. The descriptive analysis concluded with the use of measures of relative standing, which described one participant's score relative to the scores of other participants.

Before beginning the inferential analysis involving results from the MES and SIOP observations, I examined the interrater reliability of $10 \%$ of the SIOP observations that I and another individual had scored. A two-tailed Pearson Product-moment Correlation was used to examine the interrater reliability of all eight categories on each of the SIOP observations selected for interrater reliability review.

Next, the quantitative analysis involved an inferential analysis in which groups of two or more variables were compared (Creswell, 2008). In hypothesis testing, the observed value of the sample is compared to the population value to determine if there is 
no difference between the values and whether a relationship exists between the values (Creswell, 2008). The hypotheses being tested include the null hypothesis (i.e., there is no relationship between teachers' attitudes toward multiculturalism and their classroom implementation of sheltered instruction), and the alternative hypothesis (i.e., there is a relationship between teachers' attitudes toward multiculturalism and their classroom implementation of sheltered instruction). The level of significance or alpha for rejecting the null hypothesis was set to $p<.05$. Because the hypothesis is nondirectional and states only that there is a relationship between teachers' multicultural attitudes and their classroom use of sheltered instruction, a two-tailed test of significance was used to establish the rejection region of the null hypothesis (Salkind, 2004). Computing the sample statistic allows the researcher to decide if the computed $p$ value falls within the critical region (Creswell, 2008). The Pearson Product-moment Correlation was used to test the hypothesis between two interval variables (Salkind, 2004): the total MES score and total SIOP score.

The final stage of hypothesis testing determined whether to reject or fail to reject the null hypothesis (Creswell, 2008). After obtaining the $p$ value from the two-tailed test of significance, the researcher rejects the null hypothesis if $p<.05$. Because the decision to reject or accept the null hypothesis does not indicate the extent of the differences in the mean scores, confidence intervals were used to understand how large the variation might be (Creswell, 2008). Additionally, the confidence interval provided a more accurate estimate of the range of acceptable values consistent with observed data and likely to be contained in the actual population. In this study, the confidence interval was set to $95 \%$, 
indicating that $95 \%$ of the time the population value would fall between the ranges of the interval.

A two-tailed Pearson Product-moment Correlation decision at the $p<.05$ level was also used to ascertain if there was a statistically significant relationship between variables on the Demographic Questionnaire and the total SIOP score with the intention of eliminating control variables as possible explanations for the outcome (Creswell, 2008).

The next phase of the quantitative analysis focused on the CIQ results. The use of descriptive statistics provided information on how participants rated themselves in terms of their use of sheltered instruction. Measures of central tendency and variability demonstrated the overall trends in the quantitative CIQ responses, while the Kolmogorov-Smirov test provided information on the distribution of the data. Using measures of relative standing, each participant was ranked based on their responses to the quantitative section of the CIQ. The analysis of the quantitative CIQ data concluded with the use of a two-tailed Pearson Product-moment Correlation to ascertain whether there was a statistically significant relationship between participants' total SIOP score and their self-evaluation of their use of sheltered instructional techniques.

Qualitative. Qualitative data analysis involves (a) preparing and organizing the data for analysis, (b) exploring and coding the data, and (c) building description and themes from the data (Creswell, 2008). Responses to the CIQ and semi-structured interviews were typed into a word document for analysis. Because I was analyzing a small dataset (i.e., less than 500 pages of transcripts or field notes) and preferred to be close to the data, I conducted the data analysis by hand (Creswell, 2008). Additionally, 
the qualitative data analysis began after the CIQs had been completed and continued with the interview responses (Maxwell, 2005).

The process of exploring and coding the data began by examining the data to get a general sense of it, noting ideas in the margins of field notes and transcripts, reflecting on the organization of the data, and considering whether more data were needed (Creswell, 2008). Coding involved segmenting and labeling text to form descriptions of participants' concepts and beliefs, identifying broad themes, and developing theoretical concepts (Creswell, 2008; Maxwell, 2005). In this study, the coding process entailed (a) creating a start list of codes consisting of two principle categories: multiculturalism and working with diverse students (see Appendix I); (b) revising the start list of codes by eliminating codes that were not used and further subdividing the codes into more specific categories to represent participants' ideas; (c) labeling the left margins of the CIQ responses with codes; (d) rereading the CIQ responses and recoding them as necessary; and finally (e) combining the codes into broad themes and noting these in the right-hand margins of the responses. As a means of reflecting and reviewing the main concepts, themes, issues of participants' answers in the CIQ, I prepared a contact summary sheet for each participant (Miles \& Huberman, 1994). On each participant's contact summary sheet, I noted the main issues or themes, a summary of the information the participant provided for each question on the CIQ, the ideas I perceived as salient or interesting, and new questions. Questions generated at this point in the qualitative analysis were included in the semi-structured interviews.

Similar to the process used for the CIQs, the analysis of the semi-structured interviews involved multiple steps. At the conclusion of each interview, for 
approximately 10 minutes, I noted my reflection of the interview including (a) what I had learned that was specific to that person, (b) how her thoughts and ways of approaching the instruction of diverse students related to my original hypothesis, (c) and the questions and ideas that I could explore in subsequent interviews. Once I had transcribed the interviews, I underlined main points in the participants' responses and then noted memos in the right-hand margins. I used memos for the semi-structured interviews to identify themes and their relationships (Miles \& Huberman, 1994). Themes for both the CIQ and semi-structured interviews were developed by their type, major and minor. Next, I created a case-ordered data matrix to compare the six interviewed participants across the major variables identified from the interviews: (a) multiculturalism, (b) life experiences supportive of multiculturalism, (c) observation of students, (d) sheltered instruction, and (e) learning process (Miles \& Huberman, 1994). Any contrary evidence that did not support the identified themes was reported. To provide additional rigor and insight to the study and highlight any relationship between the themes, any interconnecting themes were identified and described (Creswell, 2008; Miles \& Huberman, 1994). Finally, I used respondent validation to confirm participants' meaning in interviews and openended questionnaires (Maxwell, 2005).

\section{Representing and Reporting Findings}

Consistent with the study's explanatory mixed method design, findings from the quantitative analysis are reported first followed by the qualitative findings. Each section reports the findings from each instrument as well as any connections between results on the instruments. Each section is organized to address the research questions. The quantitative section responds to all three research questions, while the qualitative section 
focuses on question three. Finally, the findings reported in the quantitative and qualitative sections are synthesized into a third section that addresses question one from both perspectives.

Quantitative. Data gathered from the Demographic Questionnaire are reported in tables and figures to clearly describe the participants. The descriptive data are organized into tables and column charts that include measures of central tendency and variability (APA, 2002; Creswell, 2002), as well as the frequency data for the independent variables. The inferential data include the value of the test statistic, the probability of obtaining a value as extreme as or more extreme than the one obtained, and the direction of the effect (APA, 2002). The findings related to question two include a table consisting of the measures of central tendency for each section of the SIOP and for each observation, measures of variability for the two SIOP observations, and each participant's rank based on the sum of the two SIOP observations. Findings related to question three include results from the Pearson Product-moment Correlation test, tables of participants' selfrating of their use of sheltered instructional techniques and comparison of their self-rating with their total SIOP observation score, measures of central tendency and variability for the quantitative section of the CIQ, as well as each participant's rank on the quantitative section of the CIQ and rank on all three quantitative measure (i.e., MES, SIOP 1-2, and CIQ).

Qualitative. The qualitative data collected for this study are used to answer question three regarding the relationship between teachers' multicultural attitudes and their classroom implementation of sheltered instructional techniques. Tables and figures are used to illustrate the relationship between themes drawn from the CIQ and semi- 
structured interview analyses (Creswell, 2008). The questions that arose from the analysis process are reported in text. Finally, a case-ordered data matrix is used to compare the principle themes across the six participants interviewed (Miles \& Huberman, 1994). 


\section{Chapter 4}

\section{Results}

\section{Question 1}

What are teachers' levels of multicultural efficacy as measured by the Multicultural Efficacy Scale? This first research question was answered by the descriptive analysis of the MES (see Tables 4-5).

Table 4

Descriptive Statistics for the Multicultural Efficacy Scale Scores-Central Tendency $(N=15)$

\begin{tabular}{lccc} 
Subscale & Mean & Median & Mode \\
\hline Experience with diversity & 21.07 & 23.00 & 25.00 \\
Attitude about diversity & 24.27 & 24.00 & 24.00 \\
$\begin{array}{l}\text { Self-assessment of teaching efficacy } \\
\quad \text { related to diversity }\end{array}$ & 64.53 & 65.00 & 65.00 \\
$\begin{array}{l}\text { Conception of multiculturalism } \\
\text { Plion }\end{array}$ & 4.00 & 4.00 & 5.00 \\
\hline
\end{tabular}

The total possible points for each subscale of the MES in the order they appear in Table 4 are $28,28,80$, and 5 . On average, participants scored between $75-80 \%$ on each of the four subscales. The $95 \%$ confidence intervals for each subscale of the MES in order of appearance in Table 4 are [18.22, 23.92], [23.32, 25.21], [59.30, 69.77], and [3.41, 4.59], indicating a $95 \%$ certainty the population value would fall between the upper and lower bounds of each subscale. 
Table 5

Descriptive Statistics for the Multicultural Efficacy Scale Scores-Variability $(N=15)$

\begin{tabular}{|c|c|c|c|c|c|}
\hline Subscale & Variance & SD & Range & Skewness & Kurtosis \\
\hline Experience with diversity & 26.50 & 5.15 & 16.00 & -0.41 & -0.97 \\
\hline Attitude about diversity & 2.93 & 1.71 & 7.00 & -0.78 & 1.66 \\
\hline $\begin{array}{l}\text { Self-assessment of teaching } \\
\text { efficacy related to } \\
\text { diversity }\end{array}$ & 89.41 & 9.46 & 33.00 & -0.60 & -0.02 \\
\hline $\begin{array}{l}\text { Conception of } \\
\text { multiculturalism }\end{array}$ & 1.14 & 1.07 & 3.00 & -0.81 & -0.40 \\
\hline
\end{tabular}

The distribution of all the subscales of the MES was negatively skewed. The tails of the subscales' distributions were longer on the left than the right (See Appendix J), therefore the participants tended to score on the high end of each subscale (Salkind, 2004).

Kurtosis measures the extent a distribution is peaked or flat. Platykurtic distributions are relatively flat compared to a normal, bell-shaped distribution; whereas leptokurtic distributions are peaked compared to a normal distribution (Salkind, 2004). To ascertain whether the results on the MES subscales were normally distributed, I used the Kolmogorov-Smirov test. While histograms show when a sample deviates from the normal distribution, they do not show whether the deviation is statistically significant enough to be important. A significant value of $p<.05$ indicates a non-normal distribution (Field, 2000). Results from all subscales indicated a normal distribution or moderate attitudes related to (a) experience with diversity $(Z=.70 ; p=.72)$, (b) attitude about diversity $(Z=.57 ; p=.91)$, (c) self-assessment of teaching efficacy related to diversity $(Z=.72 ; p=.68)$, and $(\mathrm{d})$ conception of multiculturalism subscale 
$(Z=.90 ; p=.39)$.

Finally, I ranked each participant based on the total for subscales B through D on the MES, with the top rank being for the highest total score on the MES (see Table 6).

Table 6

Descriptive Statistics for the Multicultural Efficacy Scale Scores-Rank Based on Total Subscale B-D Scores $(N=15)$

\begin{tabular}{lrcr}
\hline Rank & Participant & Rank & Participant \\
\hline 1 & 13 & 9.5 & 9 \\
2 & 10 & 9.5 & 11 \\
3.5 & 1 & 11 & 12 \\
3.5 & 5 & 12 & 7 \\
5 & 15 & 13 & 3 \\
6 & 14 & 14 & 2 \\
7 & 8 & 15 & 6 \\
8 & 4 & & \\
\hline
\end{tabular}

\section{Question 2}

What are teachers' levels of implementation of sheltered instructional techniques in the classroom as measured by the Sheltered Instruction Observation Protocol? The second research question was answered by the descriptive analysis of the SIOP. 
Table 7

Descriptive Statistics for the Sheltered Instruction Observation Protocol Observations 1 and 2-Central Tendency $(N=15)$

\begin{tabular}{|c|c|c|c|c|c|c|}
\hline \multirow[b]{2}{*}{ Component } & \multicolumn{3}{|c|}{ Observation 1} & \multicolumn{3}{|c|}{ Observation 2} \\
\hline & Mean & Median & Mode & Mean & Median & Mode \\
\hline Preparation & 11.53 & 13.00 & 14.00 & 10.20 & 9.00 & 7.00 \\
\hline Building background & 4.67 & 4.00 & 0.00 & 3.20 & 3.00 & 0.00 \\
\hline Comprehensible input & 8.73 & 9.00 & 10.00 & 8.20 & 9.00 & 10.00 \\
\hline Strategies & 6.87 & 7.00 & 0.00 & 5.40 & 6.00 & 6.00 \\
\hline Interaction & 9.27 & 9.00 & 9.00 & 8.80 & 8.00 & 5.00 \\
\hline Practice or application & 3.13 & 3.00 & 2.00 & 4.20 & 4.00 & 3.00 \\
\hline Lesson delivery & 8.67 & 11.00 & 11.00 & 8.67 & 10.00 & 7.00 \\
\hline Review or assessment & 7.40 & 8.00 & 10.00 & 6.60 & 6.00 & 6.00 \\
\hline Total percentage & 50.47 & 57.00 & 63.00 & 47.27 & 46.00 & 40.00 \\
\hline
\end{tabular}

Note. Boldface numbers indicate multiple modes exist. The smallest value is shown.

The total points possible for each section of the SIOP vary and are reported in order of appearance in Table 7 beginning with Preparation: 24, 12, 12, 12, 16, 12, 16, and 16 (Echevarría et al., 2008). The average total performance of the sample for both observations was $48.87 \%$. The $95 \%$ confidence intervals for each section of the SIOP and the total percentage score in order of appearance in Table 7 are $[9.52,13.55],[2.73$, 6.60], [7.45, 10.01], [4.70, 9.03], [7.72, 10.81], [2.05, 4.22], [6.59, 10.75], [5.72, 9.08], and $[41.64,59.30]$, indicating a $95 \%$ certainty the population value would be between the upper and lower bounds for each section's score. 
Table 8

Descriptive Statistics for the Sheltered Instruction Observation Protocol Observation 1Variability $(N=15)$

\begin{tabular}{lrrrrr}
\hline Component & & & & & \\
& Variance & SD & Range & Skewness & Kurtosis \\
\hline Preparation & 13.27 & 3.64 & 13.00 & -0.59 & -0.51 \\
Building background & 12.24 & 3.50 & 10.00 & -0.01 & -1.44 \\
Comprehensible input & 5.35 & 2.31 & 8.00 & -1.74 & 2.55 \\
Strategies & 15.27 & 3.91 & 12.00 & -0.46 & -0.65 \\
Interaction & 7.78 & 2.79 & 10.00 & 0.37 & -0.23 \\
Practice or application & 3.84 & 1.96 & 7.00 & 0.64 & -0.15 \\
Lesson delivery & 14.10 & 3.75 & 13.00 & -1.19 & 0.52 \\
Review or assessment & 9.26 & 3.04 & 9.00 & -1.20 & 0.26 \\
Total percentage & 254.27 & 15.95 & 50.00 & -0.73 & -0.78 \\
& & & & & \\
\hline
\end{tabular}

Table 9

Descriptive Statistics for the Sheltered Instruction Observation Protocol Observation 2Variability $(N=15)$

\begin{tabular}{lrrrrr}
\hline Component & Variance & SD & Range & Skewness & Kurtosis \\
& & & & & \\
\hline Preparation & 23.62 & 4.86 & 17.00 & 0.49 & -0.43 \\
Building background & 9.46 & 3.08 & 9.00 & 0.48 & -0.94 \\
Comprehensible input & 4.31 & 2.08 & 6.00 & -0.42 & -1.44 \\
Strategies & 11.67 & 3.42 & 12.00 & 0.11 & -0.33 \\
Interaction & 18.46 & 4.30 & 13.00 & 0.13 & -1.36 \\
Practice or application & 5.17 & 2.27 & 7.00 & 0.14 & -1.24 \\
Lesson delivery & 17.38 & 4.17 & 16.00 & -0.30 & -0.06 \\
Review or assessment & 5.97 & 2.44 & 10.00 & 1.32 & 2.54 \\
Total percentage & 249.92 & 15.81 & 57.00 & 0.16 & -0.63 \\
& & & & & \\
\hline
\end{tabular}

For the first SIOP observation, results from the Kolmogorov-Smirov test with the significance level set to $p<.05$ indicated a normal distribution for all sections: (a) preparation $(Z=.84 ; p=.48)$, (b) building background $(Z=.63 ; p=.82),(\mathrm{c})$ 
comprehensible input $(Z=1.08 ; p=.19)$, (d) strategies $(Z=.47 ; p=.98)$, (e) interaction $(Z=.50 ; p=.96),(\mathrm{f})$ practice or application $(Z=.75 ; p=.63),(\mathrm{g})$ lesson delivery $(Z=1.29 ; p=.07),(\mathrm{h})$ review or assessment $(Z=.96 ; p=.32)$, and (i) total percentage $(Z=.82 ; p=.51)$.

Results from the Kolmogorov-Smirov test on the second sheltered instruction observation demonstrated a normal distribution for all sections (a) preparation $(Z=.55 ; p=.93),(\mathrm{b})$ building background $(Z=.71 ; p=.69),(\mathrm{c})$ comprehensible input $(Z=.80 ; p=.54),(\mathrm{d})$ strategies $(Z=.63 ; p=.82),(\mathrm{e})$ interaction $(Z=.56 ; p=.91),(\mathrm{f})$ practice/application $(\mathrm{Z}=.65 ; \mathrm{p}=.79),(\mathrm{g})$ lesson delivery $(\mathrm{Z}=.62 ; p=.84),(\mathrm{h})$ review or assessment $(Z=1.02 ; p=.25)$ and (i) total percentage $(Z=.56 ; p=.92)$.

Each participant's rank based on the sum of two SIOP observations is displayed in Table 10. The top rank equals the highest score on the summed sheltered observations. Of the 15 participants, only five scored over $60 \%$ on each of the two SIOP lessons. None of the participants scored over $70 \%$ on each of the SIOP lessons.

Table 10

Descriptive Statistics for Sheltered Instruction Observation Protocol-Rank Based on the Sum of the Two Observations $(N=15)$

\begin{tabular}{lrcr}
\hline Rank & Participant & Rank & Participant \\
\hline 1 & 4 & 8.5 & 12 \\
2 & 11 & 10 & 10 \\
3.5 & 3 & 11 & 8 \\
3.5 & 7 & 12 & 15 \\
5 & 9 & 13 & 13 \\
6 & 14 & 14 & 1 \\
7 & 6 & 15 & \\
8.5 & 5 & & \\
\hline
\end{tabular}




\section{Question 3}

What is the relationship between teachers' attitudes about multiculturalism as measured by the MES and their level of implementation of sheltered instructional techniques in the classroom as measured by the SIOP? To answer the third research question, I used a combination of descriptive statistics and correlational analyses.

A two-tailed Pearson Product-moment Correlation test of the total SIOP score and total MES score on subscales B through D showed no statistically significant relationship $(r=-.34 ; p=.22)$. Significance results for the highest scoring participants on the SIOP (i.e., scored a total of 123 or more) were similar to the group as a whole $(r=.75 ; p=.25)$, while the significance results for the lowest scoring participants (i.e., scored below 123 on the SIOP) were $(r=-.26 ; \mathrm{p}=.43)$. Therefore, I initially accepted the null hypothesis that there is no statistically significant relationship between teachers' attitudes about multiculturalism and their use of sheltered techniques.

However, the estimate of the Pearson Product-moment Correlation $(r)$ indicated a relationship between participants' attitudes about multiculturalism and their use of sheltered techniques, depending on how high they scored on the SIOP observations. The $r$ for the participants as a group was $-.34, .49$ for the highest five SIOP scorers, and .75 for the highest four SIOP scorers. A summary of the correlational results for the Total SIOP and MES subscales B through D are represented in Table 11. 
Table 11

Two-tailed Pearson Product-moment Correlation Test of the Total SIOP and MES Scores on Subscales B through D

\begin{tabular}{|c|c|c|c|}
\hline Sample & $\mathrm{N}$ & $\begin{array}{l}\text { Pearson Correlation } \\
(r)\end{array}$ & $\begin{array}{l}\text { Significance } \\
\text { (small sample size) }\end{array}$ \\
\hline All participants & 15 & -.34 & \\
\hline $\begin{array}{l}\text { Participants who } \\
\text { scored } 121 \text { or } \\
\text { above for the } \\
\text { total SIOP } \\
\text { score }\end{array}$ & 5 & .49 & \\
\hline $\begin{array}{l}\text { Participants who } \\
\text { scored } 123 \text { or } \\
\text { above for the } \\
\text { total SIOP } \\
\text { score }\end{array}$ & 4 & .75 & \\
\hline $\begin{array}{l}\text { Participants who } \\
\text { scored } 122 \text { or } \\
\text { below for the } \\
\text { total SIOP } \\
\text { score }\end{array}$ & 11 & -.26 & \\
\hline
\end{tabular}

Therefore, I rejected the null hypothesis for the highest four SIOP performers, based on the estimate of the Pearson Product-moment Correlation and accepted the null hypothesis for the lowest 11 SIOP performers.

Next, using a two-tailed Pearson Product-moment Correlation test chosen as the .05 level, I examined whether there was a statistically significant relationship between the variables on the Demographic Questionnaire and the total SIOP score. None of the variables on the Demographic Questionnaire were found to have a statistically significant relationship with the total SIOP score. I found the following results to be of particular interest in that they were not statistically significant, but I thought they would support 
teachers with using sheltered instruction: (a) specializations or majors related to English language learners $(r=.09 ; p=.76)$, (b) completed endorsements related to teaching English language learners $(r=.11 ; p=.70$, (c) total hours of sheltered training $(r=-.20 ; p=.48,(\mathrm{~d})$ personal study of sheltered techniques $(r=-.43 ; p=.11)$, and $(\mathrm{e})$ number of years teaching at least one English language learner $(r=.08 ; p=.77)$. Additionally, there was a negative relationship between the total SIOP score and participants' total hours of sheltered training $(r=-.20 ; p=.48)$ and personal study of sheltered techniques $(r=-.43 ; p=.11)$.

The two-tailed Pearson Product-moment Correlation showed statistically significant negative relationships at the .05 level for the highest four SIOP scorers between their total SIOP scores and the following (a) their socioeconomic status as adults $(r=-.99 ; p=.02)$ and (b) completed endorsements related to teaching English language learners $(r=-.99 ; p=.02)$. However, there were no statistically significant relationships between the variables on the Demographic Questionnaire and the lowest SIOP scorers. For the lowest SIOP scorers, there was a negative relationship between the total SIOP score and (a) completed endorsements related to teaching English language learners $(r=-.01 ; p=.99),(\mathrm{b})$ the total hours of participants' sheltered training $(r=-.06$; $p=.87)$, and (c) participants personal study of sheltered techniques $(r=-.30 ; p=.38)$. Additionally, for the participants as a whole, the highest four SIOP scorers, and the lowest 11 SIOP scorers, there were no statistically significant relationships between MES and SIOP scores $(r=-.34 ; p=.22),(r=.75 ; p=.25)$, and $(r=-.26 ; p=.43)$.

Question six of the CIQ prompted participants to rate their frequency of implementation of each sheltered instruction component. Results in Table 12 indicated 
the majority of participants believed they used sheltered instructional techniques most of the time. Of the eight sheltered lesson components, four were noteworthy in that $80 \%$ or more of the participants believed they always used them in the classroom. The four areas of focus included background knowledge, strategies, interaction, and practice or application. Lessons in which background knowledge is built contain concepts that are explicitly linked to students' background experiences, past learning that is connected to new concepts, and an emphasis on key vocabulary (Echevarría et al., 2008). During the SIOP observations, it was common for participants to omit at least one of the three characteristics of building background. As a group, participants scored on average $32.8 \%$ on this component across the two observations.

Always using strategies in a sheltered lesson requires the teacher to provide students multiple opportunities to use learning strategies, consistently use scaffolding techniques to support student understanding, and ask a variety of questions to promote students' use of higher-order thinking skills (Echevarría et al., 2008). A lack of learning strategies was evident during most participants' SIOP observations. On average, participants as a group scored $51 \%$ on this component across the two observations.

Interaction is a key component of sheltered instruction lessons because it provides English language learners with frequent opportunities to discuss and use elaborated responses related to lesson concepts affording students necessary English practice. In the interaction component, language and content objectives are partially supported by how students are grouped. Additionally, key concepts are clarified as needed with the first language and sufficient wait time for students' responses is provided (Echevarría et al., 2008). During the SIOP observations, I noticed students were often seated in groups; 
however, the assigned task did not require them to communicate with each other. In addition, when responding to questions, students often gave answers that lacked depth and were not elaborate. On average, participants as a group scored $56.4 \%$ on the interaction component across the two observations.

Practice or application in the SIOP model is a characterized by the use of handson materials or manipulatives for student practice of new content knowledge - activities that require the application of content and language knowledge, and activities that integrate all language skills (Echevarría et al., 2008). During the observed lessons, I found hands-on materials or manipulatives were often absent from observed lessons. Similarly, activities tended to focus on only one or two language skills, rather than an integration of all four. On average, participants as a group scored $30.5 \%$ on this area of instruction across the two observations. 
Table 12

Participants' Description of How Often They Use Sheltered Instructional Techniques from the Quantitative Section of the Classroom Instruction Questionnaire $(N=15)$

\begin{tabular}{|c|c|c|}
\hline Component & Frequency & Percent \\
\hline \multicolumn{3}{|l|}{ Preparation } \\
\hline Never or almost never & 0 & 0.0 \\
\hline Sometimes & 9 & 60.0 \\
\hline Always & 6 & 40.0 \\
\hline \multicolumn{3}{|l|}{ Building background } \\
\hline Never or almost never & 0 & 0.0 \\
\hline Sometimes & 3 & 20.0 \\
\hline Always & 12 & 80.0 \\
\hline \multicolumn{3}{|l|}{ Comprehensible input } \\
\hline Never or almost never & 0 & 0.0 \\
\hline Sometimes & 6 & 40.0 \\
\hline Always & 9 & 60.0 \\
\hline \multicolumn{3}{|l|}{ Strategies } \\
\hline Never or almost never & 0 & 0.0 \\
\hline Sometimes & 2 & 13.3 \\
\hline Always & 13 & 86.7 \\
\hline \multicolumn{3}{|l|}{ Interaction } \\
\hline Never or almost never & 1 & 6.7 \\
\hline Sometimes & 1 & 6.7 \\
\hline Always & 13 & 86.7 \\
\hline \multicolumn{3}{|l|}{ Practice or application } \\
\hline Never or almost never & 0 & 0.0 \\
\hline Sometimes & 3 & 20.0 \\
\hline Always & 12 & 80.0 \\
\hline \multicolumn{3}{|l|}{ Lesson delivery } \\
\hline Never or almost never & 0 & 0.0 \\
\hline Sometimes & 9 & 60.0 \\
\hline Always & 6 & 40.0 \\
\hline \multicolumn{3}{|l|}{ Review or assessment } \\
\hline Never or almost never & 0 & 0.0 \\
\hline Sometimes & 5 & 33.3 \\
\hline Always & 10 & 66.7 \\
\hline
\end{tabular}


Although I selected only four sheltered instructional components as examples of how participants' perception of their instruction differed from their observational scores (see Table 12), this discrepancy was also evident among the other four components as illustrated by the comparison of participants' self-rating of sheltered instructional use and their total SIOP observational scores (see Table 13).

Table 13

Participants' Description of How Often They Use Sheltered Instructional Techniques and Their Total SIOP Observation Score Reported as Percentages $(N=15)$

\begin{tabular}{llll}
\hline Participant & $\begin{array}{l}\text { Self-rating } \\
\text { of SI Use }\end{array}$ & $\begin{array}{l}\text { Total SIOP } \\
\text { Observation } \\
\text { Score }\end{array}$ & $\begin{array}{l}\text { Difference Between } \\
\text { Self-rating and Total } \\
\text { SIOP Observation } \\
\text { Score }\end{array}$ \\
\hline 4 & & & 5 \\
11 & & 70 & 18.3 \\
3 & 75 & 63 & 7.3 \\
7 & 81.3 & 61.5 & 32.3 \\
9 & 68.8 & 61.5 & 14.5 \\
14 & 93.8 & 60.5 & 16 \\
6 & 75 & 59 & -0.7 \\
5 & 75 & 57 & 32.8 \\
12 & 56.3 & 48.5 & 39 \\
10 & 81.3 & 48.5 & 53 \\
8 & 87.5 & 47 & 50 \\
15 & 100 & 37.5 & 57.8 \\
13 & 87.5 & 36 & 58 \\
1 & 93.8 & 29.5 & 65.8 \\
2 & 87.5 & 28 & 55.8 \\
\hline
\end{tabular}

Note. Participants appear in descending order based on the total SIOP observation score.

Participants' scores on question six of the CIQ are displayed in Tables 14 and 15, while their rank on the CIQ is displayed in Table 16. 
Table 14

Descriptive Statistics for the Quantitative Section of the Classroom Instruction Questionnaire-Central Tendency $(N=15)$

\begin{tabular}{llll}
\hline Section & Mean & Median & Mode \\
\hline Preparation & 1.4 & 1.0 & 1.0 \\
Building background & 1.8 & 2.0 & 2.0 \\
Comprehensible input & 1.6 & 2.0 & 2.0 \\
Strategies & 1.87 & 2.0 & 2.0 \\
Interaction & 1.8 & 2.0 & 2.0 \\
Practice or application & 1.8 & 2.0 & 2.0 \\
Lesson delivery & 1.4 & 1.0 & 1.0 \\
Review or assessment & 1.67 & 2.0 & 2.0 \\
& & & \\
\hline
\end{tabular}

There are two total possible points for each subscale of the CIQ. The 95\% CIs for each section of the CIQ in order of appearance in Table 12 are [1.12, 1.68], [1.57, 2.03], $[1.32,1.88],[1.67,2.06],[1.49,2.11],[1.57,2.03],[1.12,1.68]$, and [1.40, 1.94]. This indicates with $95 \%$ certainty that the population value would be within the upper and lower bounds of the confidence intervals. 
Table 15

Descriptive Statistics for the Quantitative Section of the Classroom Instruction Questionnaire-Variability $(N=15)$

\begin{tabular}{lllrrr}
\hline Section & Variance & SD & Range & Skewness & Kurtosis \\
\hline Preparation & & & & & \\
Building background & 0.26 & 0.51 & 1.00 & 0.46 & -2.09 \\
Comprehensible input & 0.17 & 0.41 & 1.00 & -1.67 & 0.90 \\
Strategies & 0.26 & 0.51 & 1.00 & -0.46 & -2.09 \\
Interaction & 0.12 & 0.35 & 1.00 & -2.41 & 4.35 \\
Practice or application & 0.31 & 0.56 & 2.00 & -2.92 & 8.39 \\
Lesson delivery & 0.17 & 0.41 & 1.00 & -1.67 & 0.90 \\
Review or assessment & 0.26 & 0.51 & 1.00 & 0.46 & -2.09 \\
& 0.24 & 0.49 & 1.00 & -0.79 & -1.62 \\
\hline
\end{tabular}

Table 16

Descriptive Statistics for the Quantitative Section of the Classroom Instruction Questionnaire-Rank Based on Total Score $(N=15)$

\begin{tabular}{lccc}
\hline Rank & Participant & Rank & Participant \\
\hline 1 & 10 & 9.5 & 2 \\
3.5 & 1 & 9.5 & 5 \\
3.5 & 7 & 12 & 4 \\
3.5 & 11 & 12 & 9 \\
3.5 & 15 & 12 & 14 \\
7 & 8 & 14 & 3 \\
7 & 12 & 15 & 6 \\
7 & 13 & & \\
\hline
\end{tabular}

As previously stated, it was common for participants to state they used sheltered instruction techniques more often than was indicated by the SIOP observations.

Similarly, a two-tailed Pearson Product-moment Correlation test of the total SIOP score and total CIQ score from the quantitative section found no statistically significant 
relationship $(r=-.40 ; p=.14)$ at the .05 level. Each participant's rank on all the measures: the MES, SIOP observations, and CIQ is summarized in Table 16.

Table 17

Descriptive Statistics for all Quantitative Measures-Rank Based on Total Scores

\begin{tabular}{lccc}
\hline & \multicolumn{3}{c}{ Rank } \\
\cline { 2 - 4 } Participant & MES & SIOP 1-2 & CIQ \\
\hline 1 & 3.5 & 14 & 3.5 \\
2 & 14 & 15 & 9.5 \\
3 & 13 & 3.5 & 14 \\
4 & 8 & 1 & 12 \\
5 & 3.5 & 8.5 & 9.5 \\
6 & 15 & 7 & 15 \\
7 & 12 & 3.5 & 3.5 \\
8 & 7 & 11 & 12 \\
9 & 9.5 & 5 & 1 \\
10 & 2 & 10 & 9.5 \\
11 & 9.5 & 2 & 7 \\
12 & 11 & 8.5 & 12 \\
13 & 1 & 13 & 3.5 \\
14 & 6 & 6 & \\
15 & 5 & 12 & \\
\hline
\end{tabular}

The qualitative results of this study served to refine, extend, and explain the quantitative results of the study (Creswell, 2008). The main concepts, themes, and issues of each participant's responses to the CIQ were summarized with contact summary sheets (Miles \& Huberman, 1994). Based on the analysis of the CIQ transcripts and the contact summary sheets, I identified the following principle themes related to incorporating multicultural educational practices into content: (a) teaching content with multicultural educational practices to improve students' academic learning and (b) the need for inclusion of multicultural educational practices in content areas to prepare students for a 
multicultural world. Nine participants asserted that by developing students' tolerance and awareness of other perspectives, empathy, morality, identity, and skills for cooperating and resolving conflict, students would become prepared for a multicultural world. Therefore, the majority of the participants did not describe the importance of multiculturalism in terms of how it helped students perform academically.

According to the participants, there were several challenges to teaching content with multicultural educational practices (see Figure 12) including (a) the lack of appropriate materials, time, and teacher knowledge which created barriers to teaching content with multicultural educational practices $(n=7)$; (b) resistance from others including teachers, the community, and mainstream culture $(n=5)$; and (c) students' level of maturity as a barrier to using multicultural educational practices in the content areas $(n=2)$. For example, one participant described students giggling or laughing when multicultural topics were raised. Finally, one participant explained that there is diversity among her students' cultures and that various instructional practices are not effective for all her students. Therefore, it is difficult to make generalizations that are true for all students. 


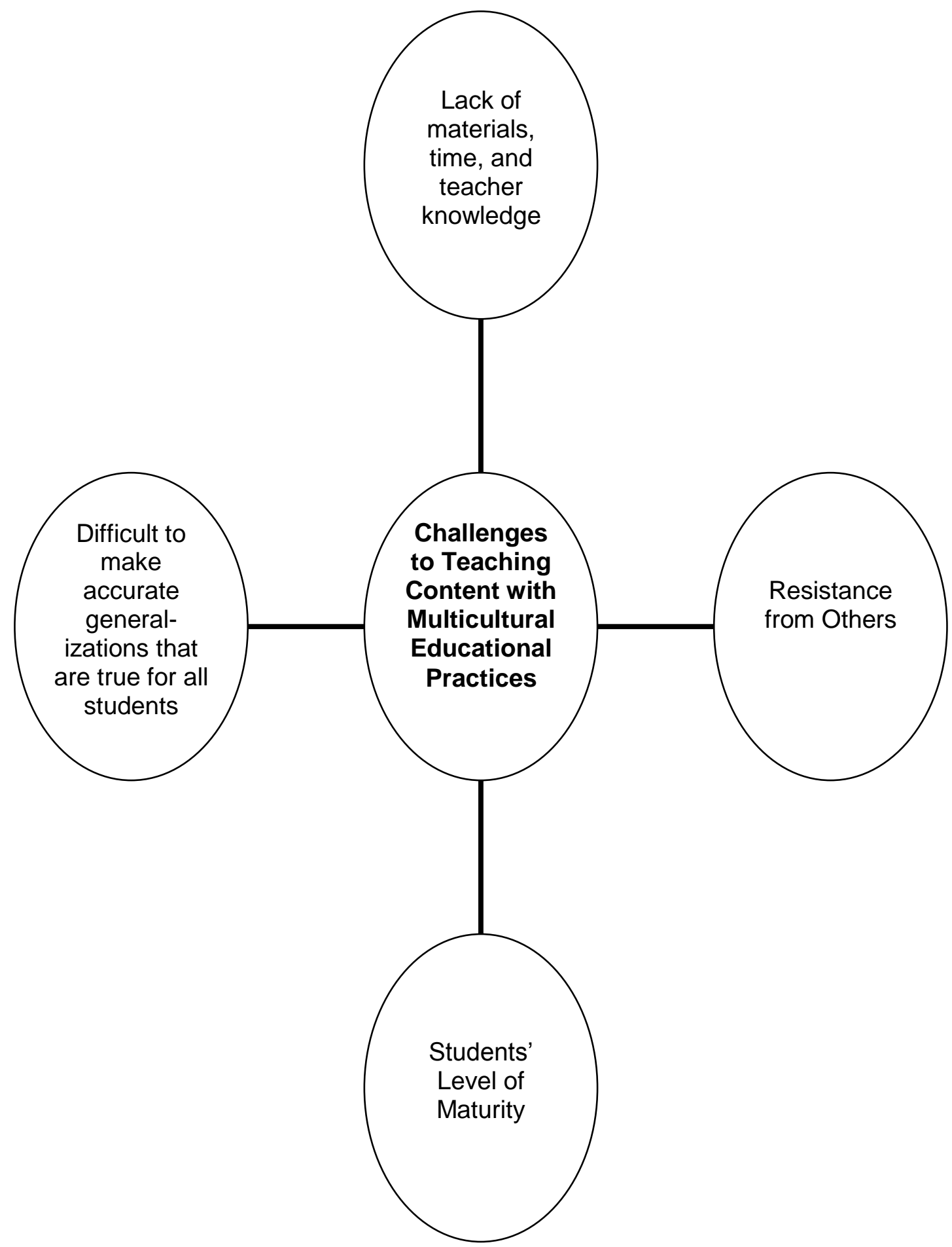

Figure 12. Participants' description of the challenges to teaching content with multicultural educational practices. 
Participants described several benefits to working with diverse students (see Figure 13) including: (a) learning about other perspectives and cultures $(n=9)$ and (b) improving instruction by making the class more interesting and motivating and requiring the teacher to tailor instruction to the students (i.e., using different strategies to improve student comprehension) $(n=4)$. Two participants did not discuss how working with diverse students was beneficial; rather, they described the benefit to the students or stated that a classroom should reflect the reality of the world.

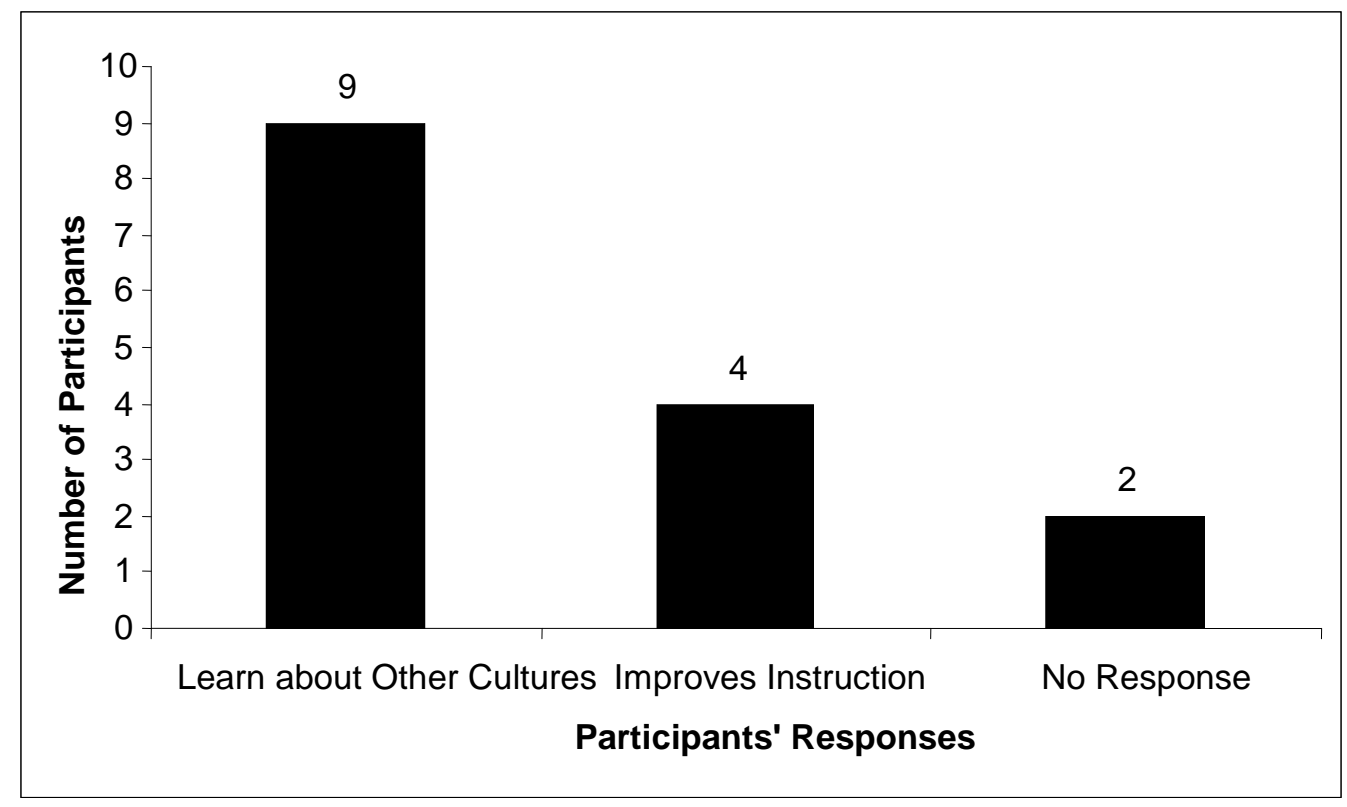

Figure 13. Participants' description of the benefits to working with diverse students.

Participants also described the challenges attributed to working with diverse students consisting of: (a) unintentionally offending students or responding to students who felt uncomfortable with teachers whose backgrounds differed from theirs $(n=5)$; (b) lacking sufficient information or skill to teach diverse students well (i.e., cultural knowledge, how to instruct students with various levels of language proficiency and ways of learning, and being able to self-evaluate and reflect to understand personal values as 
they compare to those of the students) $(n=9)$; (c) the lack of sufficient materials, time to work with individual students, and time to prepare lessons $(n=1)$; $(\mathrm{d})$ having a large number of students in classes $(n=1)$; and (e) seeing the injustice students experience and providing them encouragement to compensate $(n=1)$ (see Figure 14).

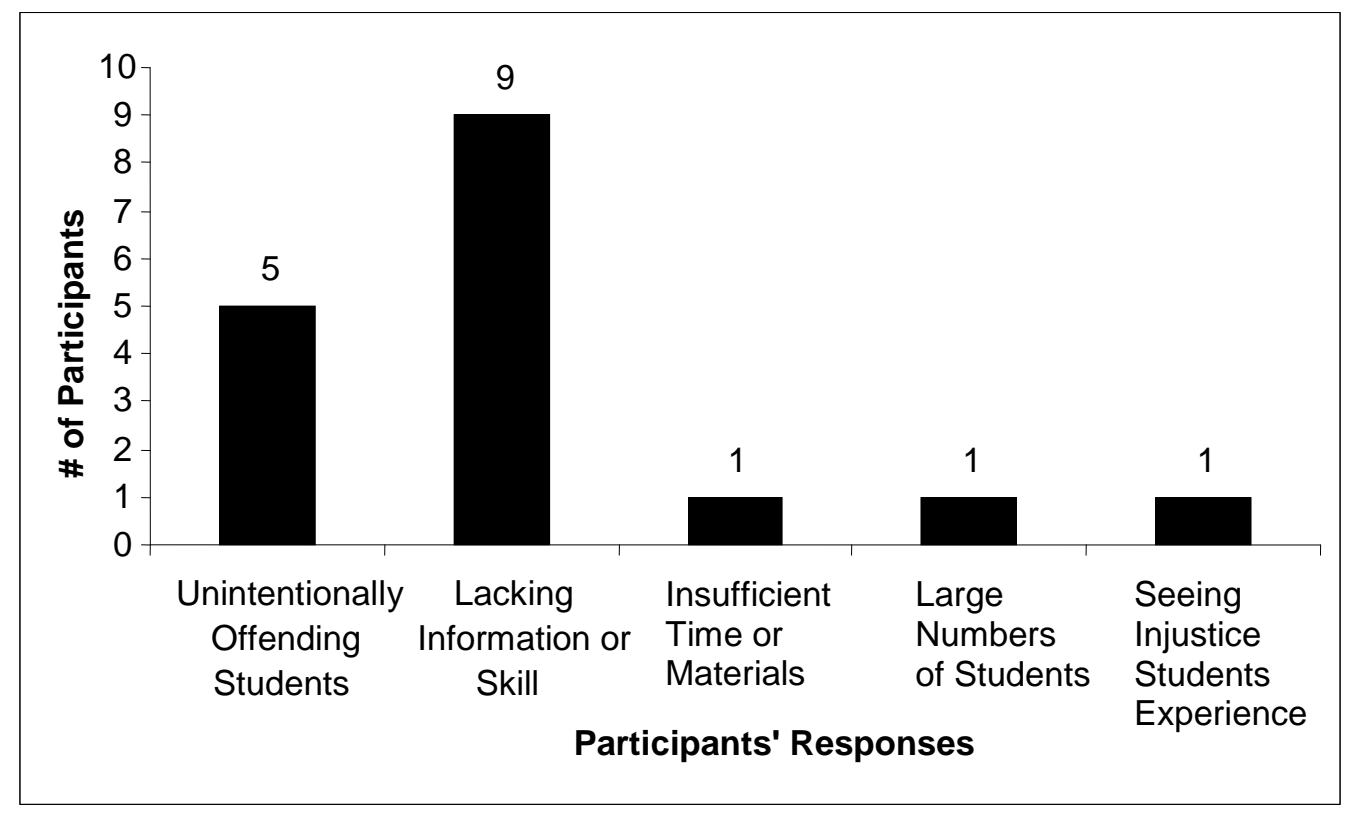

Figure 14. Participants' description of the challenges to working with diverse students.

While completing and reflecting about each participant's contact summary sheet, various questions were raised related to: (a) the knowledge and skills required to successfully implement multicultural educational practices into content, (b) the barriers to implementing multicultural educational practices in addition to time and materials, (c) identifying what caused some teachers to believe multicultural education was important, and (d) identifying possible explanations for how a participant who did not connect multicultural educational practices with student learning was able to score highly on the SIOP. 
Results from the contact summary sheets combined with the quantitative data results guided the selection of six participants to be interviewed. Based on the quantitative results, I grouped participants into those who had scored a 123 or above on the two SIOP lessons and those who had not. Then, I reviewed each participant's contact summary sheet and looked for relationships I did not expect to see when I began this research (e.g., participant talked about the importance of multicultural education in student learning, yet he or she was not one of the highest SIOP scorers). The quantitative results suggested: (a) for all the participants there was no statistically significant relationship between teachers' sheltered instructional training and their use of sheltered instruction in classrooms and (b) for most participants, there was no statistically significant relationship between their multicultural attitudes and their use of sheltered instruction in classrooms. At this point in the research, I wanted to understand the differences between those who scored a 123 or above on the SIOP lessons and those who did not.

The data analysis prompted the following questions (a) why did the SIOP performance of the majority of the participants in the study not match their own description of how often they use each sheltered component, (b) to what extent do participants' understand the complexity of teaching with sheltered techniques, and (c) is participant understanding a factor in their overestimation of how often they use sheltered techniques? Interviewing six participants helped to clarify the relationships and explain how participants could improve the implementation of sheltered techniques in their classrooms. 
I began the interviews with the highest SIOP scorer, Participant 4. I selected her as the initial interviewee to examine the reasons that might explain her relative success with sheltered instruction. Interview data indicated that central to her success were: (a) being conscious of hegemony in mainstream society and accepting multiple viewpoints, (b) believing sheltered instruction is essential to her English language learners' academic growth, (c) sustaining her learning of sheltered instruction over time, (d) being observant of students and adapting her behavior and teaching to meet their cultural, emotional, and academic needs, and (e) using self-reflection to improve her teaching.

Participant 4 referred to hegemony or the maintenance of the status quo through the use of power, domination, control, supremacy, and authority (Giroux, 2001) when discussing her opinion on the use of multicultural educational practices in content instruction. She stated, "I am open to learning about the ways that I either empower my students or possibly the things I may be doing that don't empower my students that I am maybe not aware of. Or trying to understand my own cultural background and the way it might impact students who are different than me and who aren't in the majority."

In response to a question about the role of sheltered instruction in her teaching, Participant 4 explained that using sheltered instruction made her a more coherent, better teacher and that sheltered techniques are an obvious need for her students. Having English language learners in her classroom gave her a sense of urgency to use sheltered instructional techniques. When asked what might explain some teachers' lack of sheltered instruction use after having received training, Participant 4 expressed that some teachers may feel overwhelmed with the amount of learning required to use sheltered instruction successfully in the classroom, a feeling she had experienced, but responded to 
by giving herself permission learn over time. She explained, "I was away from teaching for awhile raising my own kids... when I came back, it was kind of a whole new ball game. And, I was overwhelmed at first and...I knew I couldn't do it all. So, I ...gave myself permission to not do it all, and I think it might help if teachers kind of felt like if they viewed it [sheltered instruction teaching] as a long term thing out [learned] by the end of two or three years".

When asked if she thought being self-reflective played a role in her implementation of sheltered techniques in the classroom, Participant 4 stated, "And within that [environment where sheltered instruction is clearly necessary] my being reflective kind of has driven my implementation...Being reflective means you think about what you are doing...because you want to get better at it." She also talked about her habit of teaching a lesson at the beginning of the day, observing how students responded to the lesson, and then changing the lesson to improve student learning, so that by the end of the day the lesson may have been changed completely.

After this first interview, I proceeded with the other five interviews including two high scoring SIOP participants and three other participants who did not score highly on the SIOP observations. In addition to including the original questions of the semistructured interview protocol related to the role of multicultural education and sheltered instruction in the participants' classroom instruction and educating diverse students, I also asked participants about the role of self-reflection in their teaching and learning. I was interested in whether they experienced learning to teach with sheltered instruction as a long-term process, and whether participants felt proficient at using sheltered instructional techniques. 
Once the six interviews had been transcribed, coded, and memos had been written in the margins; I created a case-ordered descriptive matrix to compare principle themes from the interview analysis. The matrix allowed me to examine any patterns among high and low scoring SIOP participants in relationship to particular variables. I identified five principle themes to be used in the matrix: (a) multiculturalism (i.e., conscious of hegemony, connects multicultural education to student learning and social-emotional development, and is able to see from multiple perspectives); (b) life experiences are supportive of multiculturalism (i.e., support awareness of and understanding of multiple perspectives and support understanding how to effectively respond to students; (c) observation of students (i.e., adapts teaching to better fit students' needs based on observations and interactions with students); (d) importance of sheltered instruction (i.e., believes her students require it and cannot learn well without sheltered instruction and believes she has more to learn about sheltered instruction); and (e) the learning process (i.e., believes learning sheltered instruction is a long-term endeavor, uses self-reflection to learn and respond to students' academic needs).

Each participant was scored in each category with a + indicating the participant had met all of the descriptors, a $\checkmark$ indicating some of the descriptors had been met, and a - indicating that none or only one of the descriptors had been met. Each case or participant is listed in order of her rank on the SIOP observations (see Table 18). 
Table 18

Case-ordered Descriptive Matrix for Interview Data $(n=6)$

\begin{tabular}{ccllll} 
Case & Multiculturalism & $\begin{array}{l}\text { Life } \\
\text { Experiences }\end{array}$ & $\begin{array}{l}\text { Observation } \\
\text { of Students }\end{array}$ & $\begin{array}{l}\text { Sheltered } \\
\text { Instruction }\end{array}$ & $\begin{array}{l}\text { Learning } \\
\text { Process }\end{array}$ \\
\hline 4 & + & + & + & + & + \\
11 & + & + & + & + & + \\
7 & + & + & + & + & + \\
6 & $\checkmark$ & $\checkmark$ & $\checkmark$ & + & $\checkmark$ \\
1 & + & + & + & - & + \\
2 & $\checkmark$ & - & $\checkmark$ & $\checkmark$ & +
\end{tabular}

Note. Participants appear in descending order based on the total SIOP observation score.

Based on the matrix, several patterns were identified. The highest and lowest SIOP scorers met some or all of the descriptors for multiculturalism, while the highest SIOP scorers met all the descriptors for each theme. Participant 1 met all of the descriptors for multiculturalism, life experiences, and observation of students, yet was not one of the highest SIOP scorers. Participant 6 described the importance of sheltered instruction for students' learning, but did not meet each of the descriptors for the other themes. Participants 1 and 6 met one or some of the descriptors for the learning process (i.e., believes learning sheltered instruction is a long-term endeavor, uses self-reflection to learn and respond to students' academic needs). Conversely, Participant 2, who did meet all of the descriptors for the learning process described the frequency of her reflection, "I'm always self-reflecting...I will be up late at night wondering if I should've done it [lesson] another way." She noted subsequent changes to her lessons, "I'm really bad at getting ideas at the last minute. And so then, they [lesson ideas] play over and over...then, I need to do them." She also declared, "I'm not proficient at it [sheltered 
instruction] yet," which reveals her acknowledgement of the improvement she needed to make related to teaching with sheltered instructional techniques.

During their interviews, Participants 1 and 6 gave reasons why they do not selfreflect in their current teaching environment and described structures that would need to change if they were to self-reflect. When asked what she required to be able to focus more on self-reflection, Participant 1 replied, "The whole team of colleagues because I don't believe a teacher can grow by reflecting myself [herself]...sharing with teachers that have the same students in different subject areas can help me". Participant 6 asserted that as a classroom teacher, "It's very important to go back and analyze what's working and what's not... But, if you're not seeing what's working, then you don't have much to reflect on...if I get into other people's classrooms and say, 'Oh, why aren't I doing this?', then it helps me to reflect better because I get those ideas introduced...”.

During the process of respondent validation, Participant 6 clarified that for reflection to be effective, it is important for the teacher to understand what she is not proficient at, in addition to her successful practices. Additionally, she asserted, "It helps to have a mirror in a mentor or colleague that is able to point out what you are doing well and what you need to work on... and when people get into my classroom and dialogue and ask questions, that helps me stay focused." Participant 6 indicated that the dialogue and collaboration with colleagues helps maintain the momentum of improving teacher practice.

Finally, the lowest three participants range in their score for sheltered instruction. Of the three, Participant 6 was the only one to acknowledge students need sheltered 
instruction to learn. Though she met some of the descriptors in all of the other categories, she was not a high SIOP scorer.

Clear patterns between the highest and lowest SIOP scoring participants in the case-ordered descriptive matrix were not evidenced. Two of the participants asserted their practice would improve if the structure of their work environment allowed for reflection among colleagues. Because both participants stated this type of reflection would improve their practice, I think it is important to investigate the structures in schools that support or hinder reflection and whether such reflection influences teacher learning of sheltered instructional techniques.

\section{Conclusion}

In this next section, I synthesize the quantitative and qualitative results of the study to more clearly explain my understanding of whether there is a statistically significant relationship between teachers' multicultural attitudes and their level of implementation of the sheltered instructional techniques in their classrooms.

Significance results for the Pearson Product-moment Correlation $(p)$ indicated there was no statistically significant relationship between the multicultural attitudes of the participants and their use of sheltered instructional techniques. However, for the highest four SIOP scorers, the estimate of the Pearson Product-moment Correlation $(r)$ indicated there was a relationship between multicultural attitudes and the use of the sheltered instructional techniques. Results from question six on the CIQ and scores from the SIOP observations indicated the majority of the participants overestimated how often they used sheltered instructional techniques. This may be due to a lack of understanding of precisely what is involved in teaching with each of the sheltered instructional 
components. The highest four SIOP scorers are distinct from the other participants because they do have a somewhat accurate understanding of sheltered instruction as evidenced by their SIOP observation scores. The relationship between multicultural attitudes and the implementation of sheltered instruction might be true for this group because they do understand sheltered instruction enough to average at least a $61 \%$ on the SIOP observations. The other participants, however, have not developed enough of an understanding of sheltered instruction. Aptitude Measurement Interaction (K. Peterson, personal communication, February 18, 2011) may explain why the relationship between teachers' multicultural attitudes and SIOP implementation held only for the highest SIOP scorers. Multicultural attitudes may influence sheltered instruction use, but only when the teacher truly understands how to use sheltered instruction. This argument distinguishes between understanding the importance of sheltered instruction for English language learners or being open to using sheltered instruction and having the knowledge and skills to actually use the techniques with students.

As part of the qualitative data collection and analysis, three of the highest four SIOP scorers were interviewed. Results from their interviews were plotted on the caseordered data matrix. All three of them scored a + on all five categories of the matrix. Both high and low SIOP scorers met some or all of the descriptors for multiculturalism. Participant 1 met all the descriptors for multiculturalism, life experiences, and observation of students, yet was not a high SIOP scorer, which seemed to indicate these three themes did not influence sheltered instruction implementation. The two participants who met one or some of the descriptors for learning process described in their interviews how their environment posed barriers to their use of reflection to improve their practice. 
Overall, results from the case-ordered data matrix did not seem to indicate any patterns that might have explained the differences between the highest and lowest SIOP scorers. 


\section{Chapter 5}

\section{Discussion}

This chapter provides a review of the statement of the problem, the methodology used to study the problem, and a summary of the results. This review is followed by a discussion of the results including the relationship of the study to prior research, theoretical implications of the evidence, an explanation of unanticipated findings, implications for practice, and recommendations for future studies that would further investigate the validity of the proposed ideas.

\section{Review of the Study}

Statement of the problem. Due to the impact education has on people's lives, closing the achievement gap is an ethical imperative. The conditions necessary for closing the achievement gap have been identified (Manning \& Kovach, 2003), yet the teacher education and inservice professional development of most teachers does not adequately prepare them to address the needs of their ethnically, culturally, linguistically, and socioeconomically diverse students (Zeichner \& Hoeft, 1996). The problem is teachers of English language learners are often inadequately prepared to provide contentarea instruction (Echevarría et al., 2008).

Purpose of the study. The primary purpose of this study was to examine the relationship between teachers' multicultural attitudes and their use of sheltered instructional techniques in the classroom. This relationship was examined with the intention of informing practice in the field of professional development for teachers of English language learners. 
Research hypothesis and questions. Teachers who have positive attitudes toward multiculturalism including multicultural education are more likely to implement sheltered instructional techniques. This hypothesis was researched with the following questions.

1. What are teachers' levels of multicultural efficacy as measured by the Multicultural Efficacy Scale?

2. What are teachers' levels of implementation of sheltered instructional techniques in the classroom as measured by the Sheltered Instruction Observation Protocol?

3. What is the relationship between teachers' attitudes about multiculturalism as measured by the Multicultural Efficacy Scale and their level of implementation of sheltered instructional techniques in the classroom as measured by the Sheltered Instruction Observation Protocol?

Review of methodology. The explanatory mixed method design of this study involved the collection of quantitative data followed by the collection of qualitative data (Creswell, 2008). Explanatory correlational research was used to examine the relationships between the Demographic Questionnaire and the two quantitative measures used in the study: the Multicultural Efficacy Scale (MES) and the Sheltered Instruction Observation Protocol (SIOP), and the qualitative sections of the Classroom Instruction Questionnaire (CIQ) and the semi-structured interview, a qualitative instrument.

The analysis of the quantitative data began with the use of the Pearson Productmoment Correlation to determine if there were any relationships between variables on the quantitative measures (i.e., the MES, the SIOP, and question six of the CIQ, and between 
variables on the Demographic Questionnaire and variables on the quantitative measures). Next, the qualitative data were collected and analyzed. Based on the quantitative data results and the responses to the CIQ, six individuals were selected for interviewing. Once the interviews had been completed and analyzed, the relationships among data were examined with a case-ordered data matrix.

Results of the study. A two-tailed Pearson Product-moment Correlation test of the total SIOP score and total MES score on subscales B through D found no statistically significant relationship $(r=-.34 ; p=.22)$ at the .05 level. Significance results for the highest scoring participants on the SIOP (i.e., scored a total of 123 or more) were similar to the group as a whole $(r=.75 ; p=.25)$, while the significance results for the lowest scoring participants (i.e., scored below 123 on the SIOP) were $(r=-.26 ; p=.43)$. However, the estimate of the Pearson Product-moment Correlation $(r)$ indicated a relationship between participants' attitudes about multiculturalism and their use of sheltered techniques, depending on how high they scored on the SIOP observations. The $r$ for the participants as a group was $-.34, .49$ for the highest five SIOP scorers, and .75 for the highest four SIOP scorers.

A comparison of the three highest and lowest SIOP scorers across five themes on the case-ordered data matrix did not indicate any patterns that might distinguish the highest SIOP scorers from the lowest SIOP scorers. The difference between the two groups of participants may have been due to the lowest SIOP scorers' limited understanding of sheltered instruction. Aptitude Measurement Interaction (K. Peterson, personal communication, February 18, 2011), similar to Aptitude Treatment Interaction (Cronbach \& Snow, 1977), occurs when the participants' aptitude on the variable of 
focus (i.e., sheltered instruction) influences the extent to which the interaction between two variables (i.e., multicultural attitudes and sheltered instruction) can be demonstrated. The validity of subjects' self-report of multicultural attitudes depends to some degree on how good they are at the underlying phenomenon of interest (i.e., how proficient participants are at understanding, recognizing, and applying sheltered instruction). Participants can accurately report only when they clearly understand the basic idea and practice of interest. As noted, multicultural attitudes may influence sheltered instruction use, but only when teachers truly understand how to use sheltered instruction.

The combination of quantitative and qualitative results led me to accept the null hypothesis that there was no relationship between teachers' multicultural attitudes and their use of sheltered instruction in the classroom for the lowest SIOP scorers and accept the null hypothesis for the highest SIOP scorers.

My conclusion related to the null hypothesis was reached with an understanding of the limitations to the study. Teachers' description of their use of sheltered instruction and observations were the basis for evidence of implementation. However, data were not collected on participants' demonstration of multicultural attitudes and practices in the classroom. Participants were only asked to describe their attitudes on the MES. Yet, when asked to describe challenges to working with diverse students, nine participants described lacking sufficient information or skill to teach diverse students. Therefore, there may be some discrepancies between participants' classroom behavior and selfdescriptions of multicultural attitudes.

The sample consisted of individuals who were not systematically selected for the study; rather, participants volunteered to be part of the study. Therefore, the results 
cannot be generalized with confidence to the entire teaching population of the school (Creswell, 2008). Additionally, 30 participants are needed in a study for it to be considered a large enough sample for simple types of analyses (Bouma \& Ling, 2004; Creswell, 2008; Salkind, 2004). Therefore, the results from the study's statistical analysis may have been affected by the small sample of participants.

\section{Discussion of Results}

This study drew upon the research literature from the fields of multiculturalism, the education of English language learners, adult learning theory, teacher beliefs, and effective professional development for teachers. The hypothesis that teachers who have positive attitudes toward multiculturalism including multicultural education are more likely to implement sheltered instructional techniques was supported by the research literature in the fields of multicultural and English language learner education and teacher beliefs. Valuing cultural differences, affirming pluralism, and challenging discrimination in schools and society by promoting social justice are aspects of multiculturalism (NAME, 2003) that support English language learners receiving instruction that is tailored to their needs (e.g., sheltered instruction).

Multiculturalism directly opposes the system of hegemony consisting of power, domination, control, supremacy, and authority used to maintain the status quo (Giroux, 2001). Hegemonic beliefs, such as the perception of White, middle class culture and behaviors as the standard to which all other cultures and individuals should aspire, contribute to the underachievement of English language learners by informing (a) the design of inappropriate educational programs based on the inaccurate assumption that what is effective for native-English speakers will be as effective for English language 
learners (LaCelle-Peterson \& Rivera, 1994), (b) teachers' low expectations of English language learners, and (c) teachers' inaccurate perception of English language learners as low-performing native-English speakers (Waxman \& Padrón, 2002).

Teachers' beliefs influence the perceptions they have of their students and their ability to learn, and such beliefs influence the teachers' own learning. According to Richardson (1996), beliefs significantly influence how and what preservice teachers learn. Teachers' entering beliefs continue to shape their ideas and practice if they do not develop a clear understanding of what it means to be a good teacher and have strong professional commitments (Feiman-Nemser, 2001). Unfortunately, much of the professional development teachers receive once they are working in schools does not use principles for optimal learning, making it more difficult for them to learn effective instructional practices upon leaving the university. Examples of optimal learning embedded in professional development include (a) instruction that improves teachers' knowledge and pedagogical skills and (b) collaboration among teachers and between teachers and principals to solve problems related to teaching and learning. Professional development for teachers frequently is not (a) learner centered in which the teachers are asked what type of support would be most helpful to them; (b) knowledge centered in which a technique is introduced within the context of why it is of benefit, how it should be used, and when it is most appropriate to use; (c) assessment centered by providing teachers feedback as they use the technique in their classrooms which supports developing teachers' capacity to judge the successful transfer of the technique to classroom practice and the effect of the technique on students' achievement; and (d) 
community centered involving continued support and contact among teachers as they learn to incorporate new ideas into their teaching (National Research Council, 2000).

Results of my study did indicate a relationship between multicultural attitudes and sheltered instruction implementation for the highest SIOP scorers. During the interviews, two of the lowest scoring SIOP performers described some of the barriers to using selfreflection to improve their teaching practice, something they thought would be support their improvement as teachers. Reflection involves looking back on a experience in a way that informs practice, learning in the midst of practice, and/or making informed and intelligent decisions about what to do, when to do it, and why it should be done (Richert, 1990; Schön, 1983; Shulman, 1987). Effective teaching requires reflective, rational, and conscious decision-making. Educators who are reflective constantly test the assumptions and inferences they have made as part of their work as teachers (Brubacher, Case, \& Reagan, 1994). The importance of continually testing assumptions and inferences becomes evident when considering how different the experiences of teachers and students are in today's classrooms. The majority of teachers in the U.S. are White, middle class, and female (National Education Association, 2003; Strizek, Pittsonberger, Riordan, Lyter, \& Orlofsky, 2006); whereas students are increasingly culturally, linguistically, and ethnically diverse, as well as poor (Garofano \& Sable, 2008; Lindholm-Leary, 2000; Tang, Sable, \& Hoffman, 2009). As described previously, entire programs for English language learners are based on the inaccurate assumption that the academic needs of native English speakers and English language learners are the same (Waxman \& Padrón, 2002). Individuals change their thinking by critically reflecting on the assumptions, beliefs, values, and perspectives they previously had not examined. When becoming 
more reflective, individuals' capacity to entertain alternative contexts for understanding is improved (Mezirow, 2000). Poor practice continues when teachers are not aware of their effectiveness and do not engage in the process of discovery to become more aware (Jay, 2003). During their interviews, two of the highest SIOP scorers gave examples of their facility with entertaining alternative contexts when they described their practice of changing lessons based on the responses of their students. A lesson taught one way at the beginning of the day could be completely different by the end of the day.

Although effective teaching requires reflection (Brubacher, Case, \& Reagan, 1994), structures within schools make it difficult for teachers to engage in reflection (Jay, 2003; Richert, 1991). Based on her case study of four National Board Certified teachers and their use of reflection, Jay (2003) asserted that conditions in the school that supported reflection determined whether reflection took place, provided teachers' intention to reflect was also present. Some of the barriers within the school that discouraged teachers to engage in reflection included (a) a climate that devalued or criticized reflection, (b) lack of capacity and materials for reflection, (c) lack of time, and (d) lack of common teaching features among staff. Similarly, Dollese (1992) commented on the infrequency with which individuals reflect on teaching or about themselves, and that reflection is not sufficiently encouraged in preparation programs or inservice programs. Similarly, Jay (2003) contended, "For schools to benefit from a reflective stance to teaching, structures must be put into place that raise expectations, foster trust, and value continuous improvement" (p. 126). The need to be persons of action is ingrained in many teachers (Dollese, 1992). 
Recommendations for further study. Additional studies are needed to learn more about the differences between teachers who score highly on the SIOP and those who do not. Therefore, Aptitude Measurement Interaction should be examined among participants who have demonstrated the ability to score at least a $61 \%$ on the SIOP. Moreover, it is important to investigate the role of reflection in teacher learning as some participants described how they needed school structures to support their reflection with their colleagues to improve their practice.

Future studies could use an explanatory mixed method design in which quantitative data are collected and analyzed prior to the collection and analysis of the qualitative data (Creswell, 2008). Explanatory mixed method studies have the benefit of using both types of data to examine completely the research problem. The quantitative data are used to establish the general idea of the research problem, while the qualitative data are used to refine or further explain the general idea (Creswell, 2008).

Future studies could incorporate the Demographic Questionnaire used in the original study as well as the following quantitative measures: (a) the MES, (b) a multicultural classroom observation protocol that includes an analysis of the materials used during the lesson, (c) a survey measuring participants' use of reflection in their own learning and teaching practice, (d) the SIOP for classroom observations, and (e) question six of the CIQ used in the original study which asks participants to rate themselves on how often they engage in various sheltered instructional practices. The following qualitative measures could be used in future studies (a) the second part of the CIQ used in the original study consisting of open-ended questions revised to include questions about the extent reflection plays a role in teachers' instructional practice in general and use of 
sheltered instruction specifically and (b) semi-structured interviews which include questions about how participants learned to use sheltered instruction and the role reflection did or did not play in their practice of sheltered instruction.

Finally, to improve the extent to which future studies could be generalized to the larger population at the research site, at least 30 participants need to be involved. Thirty or more participants would be an adequate sample size for conducting simple statistical analyses (Bouma \& Ling, 2004; Creswell, 2008; Salkind, 2004).

Implications for practice. Research has clearly identified the practices that contribute to high quality professional development for teachers (Darling-Hammond \& McLaughlin, 1995; Díaz-Maggioli, 2004; Sparks, 2002). This caliber of professional development: (a) focuses on deepening teachers' content knowledge and pedagogical skills; (b) includes opportunities for dialogue, practice, research, and reflection; (c) is embedded in educators' work and takes place during the school day; (d) is sustained over time with adequate support systems (e.g., observation, modeling, coaching, and problem solving); (e) is founded on a sense of collegiality and collaboration among teachers and between teachers and principals in solving important problems related to teaching and learning; and (f) uses andragogical instruction.

To be effective, future professional development at the research site needs to include each of the indicators of quality professional development described by DarlingHammond and McLaughlin (1995), Díaz-Maggioli (2004) and Sparks (2002). It also needs to be differentiated for the beginning to intermediate level sheltered instruction teachers at the research site. When professional development does not incorporate each 
of the quality indicators in its design, teachers struggle to reach their potential and students do not receive the quality instruction they rely on to access future opportunities. 


\section{REFERENCES}

Alfred, M. V. (2003). Sociocultural contexts and learning: Anglophone Caribbean immigrant women in U.S. postsecondary education. Adult Education Quarterly, 53(4), 242-260. doi:10.1177/07417136003254028

American Psychological Association. (2002). Publication manual of the American Psychological Association ( $5^{\text {th }}$ ed.). Washington, DC: Author.

Anderson, G. L., Bently, M., Gallegos, B., Herr, K., \& Saavedra, E. (1998). Teaching within/against the backlash: A group dialogue about power and pedagogy in the 1990s. In R. Chávez \& J. O’Donnell (Eds.), Speaking the unpleasant: The politics of (non)engagement in the multicultural education terrain (p. 274-295). Albany, NY: State University of New York.

Aristotle. (1997) Aristotle politics: Books VII and VIII (R. Kraut, Trans.). New York: Clarendon. (Original work published c.325 B.C.E.)

Bachman, L. F. (1990). Fundamental considerations in language testing. Oxford, England: Oxford University.

Banks, J. A. (2004). Multicultural education: Historical development, dimensions, and practice. In J. A. Banks \& C. A. Banks (Eds.), Handbook of research on multicultural education (2 ${ }^{\text {nd }}$ ed.) (pp. 3-28). New York: Simon \& Schuster MacMillan.

Banks, J. A., \& Banks, C. A. (1995). Introduction. In J. A. Banks \& C. A. Banks (Eds.), Handbook of research on multicultural education (pp. xi-xiv). New York: Simon $\&$ Schuster MacMillan.

Banks, J. A., \& Banks, C. A. (2007). Multicultural education: Issues and perspectives $\left(6^{\text {th }}\right.$ ed.). Hoboken, NJ: Wiley.

Banks, J. A., \& Banks, C. A. (2009). Multicultural education: Issues and perspectives ( $7^{\text {th }}$ ed.). Hoboken, NJ: Wiley.

Banks, J. A., Cookson, P., Gay, G., Hawley, W. D., Irvine, J. J., Nieto, S., ...Stephan, W. G. (2005). Education and diversity. Social Education, 69(1), 36-40.

Barone, T., Berliner, D. C., Blanchard, J., Casanova, U., \& McGowan, T. (1996). A future for teacher education: Developing a strong sense of professionalism. In J. Sikula (Ed.), Handbook of research on teacher education $\left(2^{\text {nd }}\right.$ ed.) (pp. 11081149). New York: Simon \& Schuster MacMillan.

Barry, N. H., \& Lechner, J. V. (1995). Preservice teachers' attitudes about and awareness of multicultural teaching and learning. Teaching \& Teacher Education, 11(2), 149-161. doi:10.1016/0742-051X(94)00018-2

Binder, F. M. (1974). The age of the common school, 1830-1865. New York: John Wiley \& Sons.

Birrell, J. R. (1994). Coping with the culturally unpredictable: An ethnically encapsulated beginning teacher's struggle with African-American students' ethnic behavior. The Professional Educator, 16(2), 27-37.

Bobo, L. D. (2001). Racial attitudes and relations at the close of the twentieth century. In N. J. Smelser, W. J. Wilson, \& F. Mitchell (Eds.), America becoming: Racial trends and their consequences, (1) (pp. 264-301). Washington, DC: National Academy Press. 
Boler, M., \& Zembylas, M. (2003). Discomforting truths: The emotional terrain of understanding difference. In P. P. Trifonas (Ed.), Pedagogies of difference: Rethinking education for social change (pp. 110-136). New York: RoutledgeFalmer.

Borko, H., \& Putnam, R. T. (1996). Learning to teach. In D. Berliner \& R. Calfee (Eds.), Handbook of educational psychology (pp. 673-708). New York: Simon and Schuster Macmillan.

Bouma, G., \& Ling, R. (2004). The research process $\left(5^{\text {th }}\right.$ ed.). New York: Oxford University Press.

Broder, J. M. (1998, May 3). Clinton criticizes California effort to cut bilingual education. The New York Times, Section 1, p.30, col. 3.

Brookfield, S. (1985). Self-directed learning: A critical review of the research. In S. Brookfield (Ed.), Self-directed learning: From theory to practice (pp. 5-16). San Francisco: Jossey-Bass.

Brown, H. D. (2007). Principles of language learning and teaching $\left(5^{\text {th }} \mathrm{ed}\right.$.). White Plains, NY: Pearson.

Brubacher, J. W., Case, C. W., \& Reagan, T. G. (1994). Becoming a reflective educator: How to build a culture of inquiry in the schools. Thousand Oaks, CA: Corwin.

Carter, P. L. (2005). Keepin' it real: School success beyond black and white. Oxford, England: Oxford.

Center for Adult English Language Acquisition. (2009). Second language acquisition. Retrieved from http://www.cal.org/caela/esl_resources/collections/SLA.html

Clair, N. (1993). Beliefs, self-reported practices and professional development needs of three classroom teachers with language-minority students. New York:

Teacher's College, Columbia University. (ERIC Document Reproduction Service No. ED365166)

Clair, N., \& Adger, C. T. (1999). Professional development for teachers in culturally diverse schools. Washington, DC: Center for Applied Linguistics. Retrieved from http://www.cal.org/resources/digest/profdvpt.html

Collier, V. P. (1995). Acquiring a second language for school. Directions in Language Education, 1(4). Retrieved from http://www.thomasandcollier.com/Downloads /1995_Acquiring-a-Second-Language-for-School_DLE4.pdf

Collier, V. P., \& Thomas, W. P. (2009). Educating English learners for a transformed world. Albuquerque, NM: Dual Language Education of New Mexico Fuente Press.

Corder, S. P. (1967). The significance of learner's errors. International Review of Applied Linguistics in Language Teaching, 5(4), 161-169.

Cranton, P. (2006). Understanding a promoting transformative learning: A guide for educators of adults ( $2^{\text {nd }}$ ed.). San Francisco: Jossey-Bass.

Creswell, J. W. (2002). Educational research: Planning, conducting, and evaluating quantitative and qualitative research. Upper Saddle River, NJ: Merrill Prentice Hall. 
Creswell, J. W. (2008). Educational research: Planning, conducting, and evaluating quantitative and qualitative research. Upper Saddle River, NJ: Merrill Prentice Hall.

Creswell, J. W., \& Plano Clark, V. L. (2007). Designing and conducting mixed methods research. Thousand Oaks, CA: Sage Publications.

Cronbach, L. J., \& Snow, R. E. (1977). Aptitude and instructional methods: A handbook for research interactions. New York: Irvington.

Cummins, J. (1979). Cognitive/academic language proficiency, linguistic interdependence, the optimum age question and some other matters. Working papers on bilingualism (Vol. 19) (pp. 121-129). Toronto: Ontario Institute for Studies in Education.

Cummins, J. (1981). Age on arrival and immigrant second language learning in Canada: A reassessment. Applied Linguistics, 2, 132-149.

Cummins, J. (1986). Empowering minority students: A framework for interventions. Harvard Educational Review, 56(1), 18-36.

Cummins, J. (1996). Negotiating identities: Education for empowerment in a diverse society. Ontario, CA: California Association for Bilingual Education.

Cummins, J. (2000). 'This place nurtures my spirit': Creating contexts of empowerment in linguistically-diverse schools. In R. Phillipson (Ed.), Rights to language: Equity, power, and education (pp. 249-258). Mahwah, NJ: Erlbaum.

Darder, A. (1991). Culture and power in the classroom: A critical foundation for bicultural education. Westport, CT: Bergin \& Garvey.

Darling-Hammond, L., \& McLauglin, M. (1995). Policies that support professional development in an era of reform. Phi Delta Kappan, 76(8), 597-604.

Darling-Hammond, L., \& Baratz-Snowden, J. (2007). A good teacher in every classroom: Preparing the highly qualified teachers our children deserve. Educational Horizons, 85(2), 111-132.

Delpit, L. (1988). The silenced dialogue: Power and pedagogy in educating other people's children. Harvard Educational Review, 58(3), 280-298.

Dewey, J. (1916). Democracy and education: An introduction to the philosophy of education. New York: Macmillan.

Díaz-Maggioli, G. (2003). Professional development for language teachers. Washington, DC: Center for Applied Linguistics. Retrieved from http://www.cal.org/resources/Digest/0303diaz.html

Díaz-Maggioli, G. (2004). Teacher-centered professional development. Alexandria, VA: Association for Supervision and Curriculum Development.

Dollese, R. (1992). Voices of beginning teachers: Vision and realities. New York: Teachers College Press.

Echevarría, J., \& Graves, A. (2007). Sheltered content instruction: Teaching English language learners with diverse abilities $\left(3^{\text {rd }}\right.$ ed.). Boston: Allyn \& Bacon.

Echevarría, J., Vogt, M., \& Short, D. (2008). Making content comprehensible for English learners: The SIOP model. Boston: Pearson, Allyn, \& Bacon. 
Elmore, R. F., \& Fuhrman, S. H. (2001). Holding schools accountable: Is it working? Phi Delta Kappan, 83(1), 67-72.

Ellis, R. (2008). An introduction to second language acquisition research. In R. Ellis (Ed.), The study of second language acquisition (2 ${ }^{\text {nd }}$ ed.) (pp. 5-37). Oxford, England: Oxford University.

Færch, C., \& Kasper, G. (1986). The role of comprehension in second-language learning. Applied Linguistics, 7(3), 257-274.

Feiman-Nemser, S. (2001). From preparation to practice: Designing a continuum to strengthen and sustain teaching. Teachers College Record, 103(6), 1013-1055.

Feiman-Nemser, S. (2008). Teacher learning: How do teachers learn to teach? In M. Cochran-Smith, S. Feiman-Nemser, \& D. McIntyre. Handbook of research on teacher education. New York: Routledge, Taylor, \& Francis \& The Association of Teacher Educators.

Field, A. (2000). Discovering statistics using SPSS for Windows. Thousand Oaks, CA: Sage.

Fine, M. (1991). Framing dropouts: Notes on the politics of an urban high school. Albany, NY: State University of New York.

Fine, M. (1997). Witnessing whiteness. In M. Fine, L. Weis, L. C. Powell, \& L. M. Wong (Eds.), Off white: Readings on race, power, and society (pp. 57-65). New York: Routledge.

Fine, M. (2003). Silencing and nurturing voice in an improbable context: Urban adolescents in public school. In M. Fine, \& L. Weis (Eds.), Silencing voices and extraordinary conversations: Re-imagining schools (pp. 13-37). New York: Teachers College Press.

Freire, P. (1974). Education: The practice of freedom. London: Writers and Readers.

Freire, P. (2003). Pedagogy of the Oppressed (30 ${ }^{\text {th }}$ Anniversary ed.) (M. B. Ramos, Trans.). New York: Continuum.

Friedman, A. A. (2002). What we would have liked to know: Preservice teachers' perspectives on effective teacher preparation. In Z. F. Beykont (Ed.), The power of culture: Teaching across language difference (pp. 193-217). Cambridge, MA: Harvard Education.

Friedman, J. (1978). The epistemology of social practice: A critique of objective knowledge. Theory and Society, 6(1), 75-92.

Garofano, A., \& Sable, J. (2008). Characteristics of the 100 largest public elementary and secondary school districts in the United States: 2004-2005. (NCES Publication No. 2008335). Washington, DC: National Center for Education Statistics.

Gass, S. M. (2003). Input and interaction. In C. J. Doughty \& M. H. Long (Eds.), The handbook of second language acquisition (pp. 224-255). Malden, MA: Blackwell.

Gass, S. M., \& Varonis, E. M. (1994). Input, interaction, and second language production. Studies in Second Language Acquisition Research, 16(3), 283-302. Gay, G. (2000). Culturally responsive teaching. New York: Teachers College Press. 
Giroux, H. A. (1988). Teachers as transformative intellectuals. In H. A. Giroux (Ed.), Teachers as intellectuals (pp. 121-128). New York: Bergin \& Garvey.

Giroux, H. A. (2001). Theory and resistance in education: Towards a pedagogy for the opposition (Revised \& expanded ed.). Westport, CT: Bergin \& Garvey.

Gollnick, D. M. (1995). National and state initiatives for multicultural education. In J. A. Banks (Ed.), Handbook of research on multicultural education (pp. 44-64). New York: Simon \& Schuster MacMillan.

Goodlad, J. (1990). Teachers for our nation's schools. San Francisco: Jossey-Bass.

Goodwin, A. L. (1994). Making the transition from self to other: What do preservice teachers really think about multicultural education? Journal of Teacher Education, 45(2), 119-131. doi:10.1177/0022487194045002006

Gordon, R., Kane, T. J., \& Staiger, D. O. (2006). Identifying effective teachers using performance on the job.(Discussion Paper No. 2006-01). Washington, DC: Brookings Institute.

Gorski, P. (2006). The unintentional undermining of multicultural education: Educators at the equity crossroads. In J. Landsman \& C. W. Lewis (Eds.), White teachers/diverse classrooms: A guide to building inclusive schools, promoting high expectations, and eliminating racism (pp. 61-78). Sterling, VA: Stylus.

Griego Jones, T. (2002). Relationship between pre-service teachers' beliefs about second language learning and prior experiences with non-English speakers. In L. Minaya-Rowe (Ed.), Teacher training and effective pedagogy in the context of student diversity (pp. 39-64). Greenwich, CT: Information Age Publishing.

Griffin, C. (1991). A critical perspective on sociology and adult education. In J. M. Peters, P. Jarvis, \& Associates (Eds.), Adult education: Evolution and achievements in a developing field of study (pp. 259-281). San Francisco: Jossey-Bass.

Guarino, A. J., Echevarría, J., Short, D., Schick, J. E., Forbes, S., \& Rueda, R. (2001). The Sheltered Instruction Observation Protocol. Retrieved from http://www.csulb.edu/ jechev/SIOP\%20Article\%20Guarino.pdf

Gutek, G. L. (1991). Education in the United States: An historical perspective. Needham Heights, MA: Allyn \& Bacon.

Guyton, E. M., \& Wesche, M. V. (2005). The Multicultural Efficacy Scale: Development, item selection, and reliability. Multicultural Perspectives, 7(4), 21-29. doi:10.1207/s15327892mcp0704_4

Hakuta, K., Butler, Y. G., \& Witt, D. (2000). How long does it take English language learners to attain proficiency? (Pol. Rep. 2000-1). Palo Alto, CA: University of California at Stanford: Linguistic Minority Research Institute.

Hammerness, K., Darling-Hammond, L., \& Bransford, J. (with Berliner, D., CochranSmith, M., McDonald, M., \& Zeichner, K.) (2005). How teachers learn and develop. In L. Darling-Hammond, \& J. Bransford (Eds.), Preparing teachers for a changing world: What teachers should learn and be able to do. San Francisco: Jossey-Bass. 
Hill, P. T., Roza, M., \& Harvey, J. (2008). Facing the future: Financing productive schools. Bothell, WA: Center on Reinventing Public Education, University of Washington.

Howard, G. R. (2003). We can't teach what we don't know: White teachers, multicultural schools ( $2^{\text {nd }}$ ed.). New York: Teachers College.

Jay, J. K. (2003). Quality teaching: Reflection as the heart of practice. Lanham, MD: ScarecrowEducation.

Jefferson, T. (1904). To Mr. Wythe. In A. A. Lipscomb (Ed.), The writings of Thomas Jefferson (Memorial Ed., Vol. 5) (pp. 394-399). Washington, DC: Thomas Jefferson Memorial Association.

Jensen, E. (2005). Teaching with the brain in mind ( $2^{\text {nd }}$ ed.). Alexandria, VA: Association for Supervision and Curriculum Development.

Jussim, L., Eccles, J., \& Madon, S. (1996). Social perceptions, social stereotypes, and teacher expectations: Accuracy and the quest for the powerful self-fulfilling prophecy. Advances in Experimental Social Psychology, 28, 281-388.

KewalRamani, A., Gilbertson, L., Fox, M., \& Provasnik, S. (2007). Status and trends in the education of racial and ethnic minorities. Retrieved from http://nces.ed.gov/pubs2007/minoritytrends/ind_3_10.asp

Knowles, M. S., Holton, III, E. F., \& Swanson, R. A. (2005). The adult learner: The definitive classic in adult education and human resource development $\left(6^{\text {th }} \mathrm{ed}\right.$.). Burlington, MA: Elsevier.

Krashen, S. D. (2003). Explorations in language acquisition and use: The Taipei lectures. Portsmouth, NH: Heinemann.

Kunjufu, J. (2002). Black students-middle class teachers. Chicago: African American Images.

Kvale, S., \& Brinkman, S. (2009). InterViews: Learning the craft of qualitative research interviewing ( $2^{\text {nd }}$ ed.). Thousand Oaks, CA: Sage.

LaCelle-Peterson, M. W., \& Rivera, C. (1994). Is it real for all kids?: A framework for equitable assessment policies for English language learners. Harvard Educational Review, 64(1), 55-75.

Lewis, L., Parsad, B., Carey, N., Bartfai, N., Farris, E., \& Smerdon, B. (1999). Teacher quality: A report on the preparation and qualifications of public school teachers. Washington, DC: U.S. Department of Education, Office of Educational Research and Improvement. (NCES No. 1999-080).

Lightbown, P. M., \& Spada, N. (2006). How languages are learned ( ${ }^{\text {rd }}$ ed.). New York: Oxford University.

Lindholm-Leary, K. (2000). Biliteracy for a global society: An idea book on dual language education. Washington, DC: George Washington University.

Livneh, C., \& Livneh, H. (1999). Continuing professional education among educators: Predictors of participation in learning activities. Adult Education Quarterly, 49(2), 91-106.

Long, M. H. (1996). The role of the linguistic environment in second language acquisition. In W. C. Ritchie \& T. K. Bhatia (Eds.), Handbook of second language acquisition (pp. 413-468). San Diego, CA: Academic Press. 
Lucas, T., \& Grinberg, J. (2008). Responding to the linguistic reality of mainstream classrooms. In M. Cochran-Smith, S. Feiman-Nemser, \& D. J. McIntyre (Eds.), Handbook of research on teacher education (pp. 606-636). New York: Routledge.

Lyster, R., \& Ranta, L. (1997). Corrective feedback and learner uptake: Negotiation of form in communicative classrooms. Studies in Second Language Acquisition, 20(1), 37-66.

Mackeracher, D. (2004). Making sense of adult learning ( ${ }^{\text {nd }}$ ed.). Toronto, Canada: University of Toronto Press.

Manning, J. B., \& Kovach, J. A. (2003). The continuing challenges of excellence and equity. In B. Williams (Ed.), Closing the achievement gap: A vision for changing beliefs and practices ( $2^{\text {nd }}$ ed.) (pp. 25-47). Alexandria, VA: Association for Supervision \& Curriculum Development.

Marcuse, H. (2002). One-dimensional man: Studies in the ideology of advanced industrial society (Rev. ed.). New York: Routledge Classics.

Maxwell, J. A. (2005). Qualitative research design: An interactive approach $\left(2^{\text {nd }}\right.$ ed.). Thousand Oaks, CA: Sage.

Mayer, F. (1966). Greek ideal of education. In F. Mayer (Ed.), Great Ideas of Education: Vol. 1. Bases of ancient education (pp.143-147). New Haven, CT: College \& University Press.

McClure, P., Wiener, R., Roza, M., Hill, M. (2008). Ensuring equal opportunity in public education: How local school district funding practices hurt disadvantaged students and what federal policy can do about it. Washington, DC: Center for American Progress.

McLaren, P. (2003). Life in schools: An introduction to critical pedagogy in the foundations of education ( $4^{\text {th }}$ ed.). Boston: Pearson Education.

McMillan, J. H., \& Schumacher, S. (1997). Research in education: A conceptual introduction ( $4^{\text {th }}$ ed.). New York: Longman.

Merriam, S. B. (2008). Editor's notes. Third update on adult learning theory, 119, 1-4.

Merriam, S. B., Caffarella, R. S., \& Baumgartner, L. M. (2007). Learning in adulthood: A comprehensive guide ( $3^{\text {rd }}$ ed.). San Francisco: Jossey-Bass.

Mezirow, J. (2000). Learning to think like an adult: Core concepts of Transformation Theory. In J. Mezirow (Ed.), Learning as transformation: Critical perspectives on a theory in progress (pp. 3-33). San Francisco: Jossey-Bass.

Miles, M. B., \& Huberman, A. M. (1994). Qualitative data analysis: An expanded sourcebook $\left(2^{\text {nd }}\right.$ ed.). Thousand Oaks, CA: Sage.

Minaya-Rowe, L. (2002). Introduction. In. L. Minaya-Rowe (Ed.), Teacher training and effective pedagogy in the context of student diversity (pp. xiii-xxi). Greenwich, CT: University of Connecticut.

Mohr, K. A., \& Mohr, E. S. (2007). Extending English-language learners' classroom interactions using the Response Protocol. The Reading Teacher, 60(5), 440-450.

Moll, L. (1988). Some key issues in teaching Latino students. Language Arts, 65(5), 465-472. 
Mondale, S., \& Patton, S. B. (Eds.). (2001). School: The story of American public education. Boston: Beacon.

National Association for Multicultural Education (2003). Resolutions and positions papers. Retrieved from http://www.name.org/aboutname.html\#define

National Clearinghouse for English Language Acquisition and Language Instruction Educational Programs. (2006). The growing numbers of limited English proficient students: 1994/95-2004-05. Retrieved from http://www.ncela.gwu.edu/policy/states/reports/statedata/2004LEP/ GrowingLEP_0405_Nov06.pdf

National Education Association. (2003). Status of the American public school teacher: 2000-01. Washington, DC: Author.

National Public Radio. (2002, December 9). Educating Latinos: An NPR special report. Retrieved from http://www.npr.org/programs/atc/features/2002/nov/ educating_latinos/part3.html?sc=emaf

National Research Council. (2000). How people learn: Brain, mind, experience, and school (Expanded ed.). Washington, D.C.: National Academy.

New York Times/CBS News. (2008). The New York times/CBS news poll: July 7-14, 2008. Retrieved from http://graphics8.nytimes.com/packages/pdf/politics/ 20080716_POLL.pdf

Nieto, S. (1995). From brown heroes and holidays to assimilationist agendas: Reconsidering the critiques of multicultural education. In C. E. Sleeter \& P. L. McLaren (Eds.), Multicultural education, critical pedagogy and the politics of difference (pp. 191-220). Albany, NY: State University of New York Press.

Nieto, S. (2005). Public education in the twentieth century and beyond: High hopes, broken promises, and an uncertain future. Harvard Educational Review, 75(1), 43-64.

Nieto, S., \& Bode, P. (2008). Affirming diversity: The sociopolitical context of multicultural education ( $5^{\text {th }}$ ed.). Boston: Pearson.

Oboler, S. (1995). Ethnic labels, Latino lives: Identity and the politics of (re)presentation in the United States. Minneapolis, MN: Regents of the University of Minnesota.

Ogbu, J. U., \& Simons, H. D. (1998). Voluntary and involuntary minorities: A cultural-ecological theory of school performance with some implications for education. Anthropology and Education Quarterly, 29(2), 155-188.

Orfield, G., \& Lee, C. (2005). Why segregation matters: Poverty and educational inequality. Cambridge, MA: Civil Rights Project: Harvard University.

Opinion Research Corporation. (2009). CNN/Essence research corporation poll offers insights to views of African- Americans and Whites on key political, cultural, economic issues. Retrieved from http://news.turner.com/article_display.cfm?article_id=4485

Ovando, C. J., Combs, M. C., \& Collier, V. P. (2006). Bilingual and ESL classrooms: Teaching in multicultural contexts $\left(4^{\text {th }}\right.$ ed.). New York: McGraw Hill Higher Education. 
Pang, V. O., \& Sablan, V. A. (1998). Teacher efficacy: How do teachers feel about their abilities to teach African-American students?. In M.E. Dilworth (Ed.), Being responsive to cultural differences: How teachers learn (pp. 39-58). Thousand Oaks, CA: Corwin.

Perie, M., \& Moran, R. (2005). NAEP 2004 Trends in Academic Progress: Three Decades of Student Performance in Reading and Mathematics (NCES 2005464). U.S. Department of Education, Institute of Education Sciences, National Center for Education Statistics. Washington, DC: Government Printing Office. Retrieved from, http://nces.ed.gov/nationsreportcard/pubs/2005/ 2005464.asp\#Section4

Plato, A. (n.d.a). Republic VII (G. Grube \& C. Reeve, Trans.). In J. Cooper \& D. Hutchinson (Eds.), Plato: Complete works (pp.1136-1137). Indianapolis, IN: Hackett.

Plato, A. (n.d.b). Laws VII (G. Grube \& C. Reeve, Trans.). In J. Cooper \& D. Hutchinson (Eds.), Plato: Complete works (pp.1472-1473; 1477-1488). Indianapolis, IN: Hackett.

Plato, A. (1900). Dialogues of Plato: Containing the apology of Socrates, Crito, Phaedo, and Protagoras (B. Jowett, Trans.). New York: Clarendon.

Power, E. J. (1991). A legacy of learning: A history of western education. New York: State University of New York Press.

Richardson, V. (1996). The role of attitudes and beliefs in learning to teach. In J. Sikula, T. J. Buttery, \& E. Guyton (Eds.), Handbook of research on teacher education $\left(2^{\text {nd }}\right.$ ed.) (pp. 102-119). New York: Simon \& Schuster Macmillan.

Richardson, J. (2004). Lesson study: Teachers learn how to improve instruction. Tools for Schools, 7(4), 1-6.

Richert, A. E. (1990). Teaching teachers to reflect: A consideration of programme structure. Journal of Curriculum Studies, 22(6), 509-527.

Rios, F., \& Montecinos, C. (1999). Advocating social justice and cultural affirmation: Ethnically diverse preservice teachers' perspectives on multicultural education. Equity and Excellence in Education, 32(3), 66-76.

Rist, R. C. (2000). HER Classic: Student social class and teacher expectations: The self-fulfilling prophecy in ghetto education. Harvard Educational Review, 70(3), 257-301. (Original work published 1970)

Romaine, S. (2009). Language, culture, and identity across nations. In J. A. Banks (Ed.), The Routledge international companion to multicultural education (pp. 373-384). New York: Routledge.

Rothenberg, P. S. (2002). Introduction. In P. S. Rothenberg (Ed.), White privilege: Essential readings on the other side of racism (pp. 1-5). New York: Worth.

Rothstein, R. (2004). Reforms that could help narrow the achievement gap. Policy Perspectives, 1-12. Retrieved from http://www.wested.org/online_pubs/ pp-06-02.pdf

Rudner, L. M. (1994). Questions to ask when evaluating tests. Washington, DC: ERIC Clearinghouse on Assessment and Evaluation. (ERIC Document Reproduction Service No. ED385607). 
Salkind, N. J. (2004). Statistics for people who (think they) hate statistics $\left(2^{\text {nd }}\right.$ ed.). Thousand Oaks, CA: Sage.

Sanders, W. L., \& Rivers, J. C. (1996). Cumulative and residual effects of teachers on future student academic achievement. Knoxville, TN: University of Tennessee Value-Added Research \& Assessment Center.

Sandlin, J. A. (2005). Andragogy and its discontents: An analysis of andragogy from three critical perspectives. PAACE Journal of Lifelong Learning, 14, 25-42.

Schön, D. (1983). The reflective practitioner: How professionals think in action. New York: Basic.

Shulman, L. S. (1987). Knowledge and teaching: Foundations of the new reform, Harvard Educational Review, 57(1), 1-21.

Sleeter, C. E. (1994). White racism. Multicultural Education, 1(4), 5-8, \& 39.

Sleeter, C. E. (1996). Multicultural education as social activism. Albany, NY: State University of New York.

Sleeter, C. E., \& McLaren, P. (2009). Origins of multiculturalism. In W. Au (Ed.), Rethinking multicultural education: Teaching for racial and cultural justice (pp. 17-19). Milwaukee, WI: Rethinking Schools.

Snyder, T. D., Dillow, S. A., \& Hoffman, C. M. (2009). Digest of education statistics, 2008. Retrieved from http://nces.ed.gov/pubsearch/pubsinfo.asp?pubid= 2009020

Sparks, D. (2002). Designing powerful, professional development for teachers and principals. Oxford, OH: National Staff Development Council.

Stanton-Salazar, R. D. (1997). A social capital framework for understanding the socialization of racial minority children and youths. Harvard Educational Review, 67(1), 1-40.

Strizek, G. A., Pittsonberger, J. L., Riordan, K. E., Lyter, D. M., Orlofsky, G. F. (2006). Characteristics of schools, districts, teachers, principals, and school libraries in the United States: 2003-04 (Rev. ed.) (NCES Publication No. 2006-313 REVISED). Washington, DC: National Center for Education Statistics.

$\mathrm{Su}, \mathrm{Z}$. (1997). Teaching as a profession and as a career: Minority candidates' perspectives. Teaching and Teacher Education, 13(3), 325-340. doi:10.1016/S0742-051X(96)00021-2

Suárez-Orozco, C., Suárez-Orozco, M. M., \& Todorova, I. (2008). Learning a new land: Immigrant students in American society. Cambridge, MA: Belknap Press of Harvard University.

Swain, M. (2005). The output hypothesis: Theory and research. In E. Hinkel (Ed.), Handbook of research in second language teaching and learning (pp. 471-484). Mahwah, NJ: Erlbaum.

Tang, J., Sable, J., \& Hoffman, L. (2009). Characteristics of the 100 largest public elementary and secondary school districts in the United States: 2006-2007 (NCES Publication No. 2009-342). Washington, DC: National Center for Education Statistics.

Tatum, B. D. (2003). Why are all the Black kids sitting together in the cafeteria? And other conversations about race. New York: Basic Books. 
Teachers of English to Speakers of Other Languages. (1995). TESOL statement on the education of K-12 language minority students in the United States. In TESOL resource packet: Is your school helping its language minority students meet the national educational goals?. Alexandria, VA: Author.

Teachers of English to Speakers of Other Languages. (1999). Position statement on the acquisition of academic proficiency in English. Retrieved from http://www.tesol.org/s_tesol/bin.asp?CID=32\&DID=378\&DOC=FILE.PDF

Teachers of English to Speakers of Other Languages. (2003). Standards for the accreditation of initial programs in P-2 ESL teacher education. Alexandria, VA: Author.

Téllez, K., \& Waxman, H. C. (2005). Effective professional development programs for teachers of English language learners. Philadelphia, PA: Laboratory for Student Success, The Mid-Atlantic Regional Educational Laboratory. (ERIC Document Reproduction Service No. ED497189)

Tennant, M. (2006). Psychology and adult learning ( $3^{\text {rd }}$ ed.). New York: Routledge.

Thomas, W. P., \& Collier, V. P. (1997). School effectiveness for language minority students. Washington, DC: National Clearinghouse for Bilingual Education. (ERIC Document Reproduction Service No. ED436087)

Tough, A. (1982). Intentional changes: A fresh approach to helping people change. Chicago: Follett Publishing.

United States Census Bureau. (2006). Poverty status of people by family relationship, race, and Hispanic origin: 1959 to 2006. Retrieved from http://www.census.gov/hhes/www/poverty/histpov/hstpov2.html

Valli, L. (1996). Learning to teach in cross-cultural settings: The significance of trusting relations. In F. A. Ríos (Ed.), Teacher thinking in cultural contexts (pp. 282-307). Albany, NY: State University of New York.

Vella, J. (2002). Learning to listen, learning to teach: The power of dialogue in educating adults (Rev. ed.). San Francisco: Jossey-Bass.

Villegas, A. M., \& Lucas, T. (2002). Educating culturally responsive teachers: A coherent approach. Albany, NY: State University of New York.

Walqui, A. (1999, July). Professional development for teachers of English language learners. Paper presented at an invitational conference sponsored by the National Educational Research Policy and Priorities Board, the Office of Educational Research and Improvement, and the Office of Bilingual Education and Minority Language Affairs, Washington, DC.

Washington, G. (1790). First annual message to congress: New York City, Federal Hall, Wall \& Broad Streets. Retrieved from http://www.let.rug.nl/ usa/P /gw1/speeches/gwson1.htm

Waxman, H. C., \& Padrón, Y. N. (2002). Research-based teaching practices that improve the education of English language learners. In L. Minaya-Rowe (Ed.), Teacher training and effective pedagogy in the context of student diversity (pp. 338). Greenwich, CT: Information Age.

Webb, L. D., Metha, A., \& Forbis Jordan, K. (2003). Foundations of American education $\left(4^{\text {th }}\right.$ ed.). Upper Saddle River, NJ: Merrill Prentice Hall. 
Weiner, L. (1990). Preparing the brightest for urban schools. Urban Education, 25(3), 258-273. doi: $10.1177 / 0042085990025003002$

White, L. (1987). Against comprehensible input: The Input Hypothesis and the development of second-language competence. Applied Linguistics, 8(2), 95110.

Wildman, S. M. \& Davis, A. D. (2002). Making systems of privilege visible. In P. S. Rothenberg (Ed.), White privilege: Essential readings on the other side of racism (pp. 89-95). New York: Worth.

Wirt, J., Choy, S., Rooney, P., Provasnik, S., Sen, A., \& Tobin, R. (2004). The condition of education 2004. (NCES Publication No. 2004-077). Washington, DC: National Center for Education Statistics.

Wise, T. (2009). Between Barack Obama and a hard place: Racism and white denial in the age of Obama. San Francisco: City Lights Books.

Wlodkowski, R. J. (1999). Enhancing adult motivation to learn (Rev. ed.). San Francisco: Jossey-Bass.

Wright, S. P., Horn, S. P., \& Sanders, W. L. (1997). Teacher and classroom context effects on student achievement: Implications for teacher evaluation. Journal of Personnel Evaluation in Education, 11(1), 57-67. doi:10.1023/A;1007999204543

Zeichner, K. M. (2003). Pedagogy, knowledge, and teacher preparation. In B. Williams (Ed.), Closing the achievement gap: A vision for changing beliefs and practices $\left(2^{\text {nd }}\right.$ ed.) (pp. 99-114). Alexandria, VA: Association for Supervision \& Curriculum Development.

Zeichner, K. M., \& Hoeft, K. (1996). Teacher socialization for cultural diversity. In J. Sikula (Ed.), Handbook of research on teacher education $\left(2^{\text {nd }}\right.$ ed.) (pp. 525-547). New York: Simon \& Schuster MacMillan. 


\section{APPENDIX A}

Demographic Questionnaire

TO THE PARTICIPANT: The demographic information requested below is an integral part of the research process. Please respond to each question (e.g., even if the response is "none"). Feel free to ask clarifying questions as needed. My understanding of the research problem is dependent on information that is accurate from each participant. Please be assured that this information and all of your responses on this instrument will be kept strictly confidential. Data will be reported so that identification of individuals will be impossible. Your identification number allows this information to be compared with your responses on other measurements or observations.

Identification Number:

Date:

SECTION ONE:

Gender: Female Male

Age:

Birthplace: City State/Province

Country

Racial/Ethnic Background (Please Describe):

Religious Background (Please Describe):

Physical Disabilities (If Any, Please Describe):

Parents' Education (Highest Degree/Diploma): Father Mother

Approximate Socio-Economic Status (Please Check One in Each Column): As a Child As an Adult (Current) Corresponding Annual Household Income

Lower Lower \$0-\$19,999

Lower Middle

Lower Middle

$\$ 20,000-\$ 39,999$

Middle Middle \$40,000-\$59,999

Upper Middle Upper Middle \$60,000-\$79,999 Upper Upper $\$ 80,000+$ 


\section{SECTION TWO:}

Education: Highest Completed Degree

Specialization /Major

Completed Endorsements

Sheltered Instruction Training: Number of Classes/Trainings You Have

Attended

Duration of each Class/Training (days and hours):

Training 1 ___ days___ hours

Training 2__ days __ hours

Additional Trainings __ days __ hours

Personal Study Related to Sheltered Instruction (Please describe.)

Teaching Experience: Number of Years Teaching Middle School (Include the 2008-2009 School Year)

Number of Years Teaching Classes with at Least One English Language Learner in Attendance (Include the 2008-2009 School Year)

\section{SECTION THREE:}

Languages Spoken: List the Languages You Speak at the Intermediate to Fluent Levels

Travel Outside the United States: List the Countries Outside of the U.S. to which You Have Traveled (If you have not traveled outside the U.S., please write "none" below. 


\section{APPENDIX B}

\section{Multicultural Efficacy Scale ${ }^{2}$}

TO THE PARTICIPANT: Please respond to each item using the directions provided for each section. Feel free to ask for clarification as needed. Because I am merely trying to get an accurate sense of your opinions on these topics, there are no right or wrong answers. Please be assured that this information and all of your responses on this instrument will be kept strictly confidential. Data will be reported in such a way that identification of individuals will be impossible. Your identification number allows this information to be compared with your responses on other measurements or observations.

\section{Identification Number:}

Date:

\section{SECTION A}

Definition: The authors intend the terms "diversity" and "people different from me" to include people of different races, ethnic groups, cultures, religious, socio-economic classes, sexual orientation, and physical abilities.

Directions: Please choose the word that best describes your experience with people different from you by circling the letter of the response.

1) As a child, I played with people different from me.
A) never
B) rarely
C) occasionally
D) frequently

2) I went to school with diverse students as a teenager.
A) never
B) rarely
C) occasionally
D) frequently

3) Diverse people lived in my neighborhood when I was a child growing up.
A) never
B) rarely
C) occasionally
D) frequently

4) In the past I chose to read books about people different from me.
A) never
B) rarely
C) occasionally
D) frequently

5) A diverse person was one of my role models when I was younger.
A) never
B) rarely
C) occasionally
D) frequently

\footnotetext{
${ }^{2}$ Adapted from Guyton, E. M., \& Wesche, M. V. (2005). The Multicultural Efficacy Scale: Development, item selection, and reliability. Multicultural Perspectives, 7(4), 21-29. Minor changes have been made involving formatting and the deletion of instructions asking the participant to respond using a scantron answer sheet. The content of the instrument remains unchanged.
} 
6) In the past I chose to watch TV shows and movies about people different from me.
A) never
B) rarely
C) occasionally
D) frequently

7) As a teenager, I was on the same team and/or club with diverse students.
A) never
B) rarely
C) occasionally
D) frequently

\section{SECTION B}

Directions: Respond to each statement by choosing one answer that best describes your reaction to it. Write the letter of your response to the left of the number for each question.

\section{$\begin{array}{llll}\text { Key: } & \text { A) agree strongly } & \text { B) agree somewhat } & \text { C) disagree somewhat }\end{array}$ D) disagree strongly}

8) Teachers should adapt lesson plans to reflect the different cultures represented in the classroom.

9) Teacher should provide opportunities for children to share cultural differences in foods, dress, family life, and beliefs.

10) Discussing ethnic traditions and beliefs in school leads to disunity and arguments between students from different cultures.

11) Children should be taught mostly by teachers of their own ethnic and cultural background.

12) It is essential to include the perspectives of diverse groups while teaching things about American history that are common to all Americans.

13) Curricula and textbooks should include the contributions of most, if not all, cultural groups in our society.

14) The classroom library should reflect the racial and cultural differences in the class. 


\section{SECTION C}

Directions: To the best of your knowledge, self-assess your own ability to do the various items listed below. Write the letter of your response to the left of the number for each question.

Key: $A=I$ do not believe I could do this very well.

$B=I$ could probably do this if $I$ had to, but it would be difficult for me. $\mathrm{C}=\mathrm{I}$ believe I could do this reasonably well, if I had time to prepare. $\mathrm{D}=\mathrm{I}$ am quite confident that this would be easy for me to $\mathrm{do}$.

15) I can provide instructional activities to help students to develop strategies for dealing with racial confrontations.

16) I can adapt instructional methods to meet the needs of learners from diverse groups.

17) I can develop materials appropriate for the multicultural classroom.

18) I can develop instructional methods that dispel myths about diverse groups.

19) I can analyze instructional materials for potential stereotypical and/or prejudicial content.

20) I can help students to examine their own prejudices.

21) I can present diverse groups in our society in a manner that will build mutual respect.

22) I can develop activities that increase the self-confidence of diverse students.

23) I can provide instruction showing how prejudice affects individuals. 


\section{Key: $A=I$ do not believe I could do this very well.}

$B=I$ could probably do this if $I$ had to, but it would be difficult for me. $\mathrm{C}=\mathrm{I}$ believe that I could do this reasonably well, if I had time to prepare. $D=I$ am quite confident that this would be easy for me to do.

24) I can plan instructional activities to reduce prejudice toward diverse groups.

25) I can identify cultural biases in commercial materials used in teaching.

26) I can help students work through problem situations caused by stereotypical and/or prejudicial attitudes.

27) I can get students from diverse groups to work together.

28) I can identify school practices that may harm diverse students.

29) I can identify solutions to problems that may arise as the result of diversity.

30) I can identify the societal forces which influence opportunities for diverse people.

31) I can identify ways in which various groups contribute to our pluralistic society.

32) I can help students take on the perspective of ethnic and cultural groups different from their own.

33) I can help students view history and current events from diverse perspectives.

34) I can involve students in making decisions and clarifying their values regarding multicultural issues. 


\section{Note: The following item is different from the others in this section.}

35) Choose the position that most closely reflects your strongest beliefs about teaching. Circle the letter of your response.

A=If every individual learned to accept and work with every other person, then there would be no intercultural problems.

$\mathrm{B}=$ If all groups could be helped to contribute to the general good and not seek special recognition, we could create a unified America.

$\mathrm{C}=\mathrm{All}$ cultural groups are entitled to maintain their own identity.

$\mathrm{D}=\mathrm{All}$ cultural groups should be recognized for their strengths and contributions.

$\mathrm{E}=$ Some groups need to be helped to achieve equal treatment before we can reach the goals of a democratic society. 


\section{APPENDIX C \\ Permission to Use the Multicultural Efficacy Scale}

\section{A DODICI}

From: $\quad$ "Ediguyton1"

To: $\quad$ "A DODICl"

Sent: $\quad$ Monday, August 11, 2008 8:11 PM

Subject: Re: Research Related to the Professional Development of Teachers of English Language Learners

No one else.

This version is the only one I know of, although some people have adapted it.

No other validity/reliability studies as far as I know.

I retired soon after the publication and have done no morc work with it duc to illness but have had pcople contact me about using it. II I still have their correspondence, I'll send it to you.

Edi Guyton

On $\Lambda$ ug 11,2008 , at 8:50 PM, " $\Lambda$ DODICI"

wrote:

Hcllo Dr. Guyton,

Thank you for granting me permission to use the Ml\$S. I will definitely share my results with you.

Would you mind answering a lew questions I have about the MES?

1. Is there anyone else I need to request permission from before I use the MES in my study'? Martin Wesche was the coauthor of the article I read regarding the MES. Should I also conlact him? If so, do you know how I could reach him?

2. The inlormation I have about the MES comes from the 2005 article The Multicultural Ellicacy

Seale: Development, item selection, and reliability published in Mulicullural Perspectives.

Is there a more recent version of the MES?

llave any more reliability or validity studies been done on the Ml:S?

Sincerely,

Adria Dodici

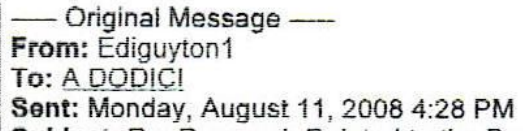

Subject: Re: Research Related to the Professional Development of Teachers of English Language Leamers

Hello. I am glad for you to use the MES for your study. I would be interested in your results. I hope you have an intcresting and informative journcy.

Fdi Cruyton 
Hello Dr. Guyton,

My name is Adria Dodici. I am a doctoral student at Portland State Universily in Portland, OR and am beginning the process of writing my dissertation. I have read about the Multicultural lifficacy Scale and would like to use it in my study. I tried contacting you at Creorgia State University, but learned you had retired. Your colleague, Dr. Rangel, suggested I try e-mailing you at the yahoo account.

In the attached letter, I explain my proposed study and how it became of interest to me. Thank you for considering this request.

Sinecrely,

Adria Dodici

$<$ Ml:S Permission I etter,doc 


\section{APPENDIX D}

\section{Sheltered Instruction Observation Protocol}

\section{Figure 3.2 The Sheltered Instruction Observation Protocol (SIOP)}

Observer:
Date:
Grade:
Class:

Teacher:

School:

ESL level:

Class:

Lesson: Multi-day Single-day (circle one)

Directions: Circle the number that best reflects what you observe in a sheltered lesson. You may give a score from $0-4$. Cite under "Comments" specific examples of the behaviors observed. Total Score: $\square$ \% Score: $\square$ Tape \#:

\section{Preparation}

1. Content objectives clearly defined for students

2. Language objectives clearly defined for students

3. Content concepts appropriate for age and educational background level of students

4. Supplementary materials used to a high degree, making the lesson clear and meaningful (graphs, models, visuals)

5. Adaptation of content (e.g., text, assignment) to all levels of student proficiency

6. Meaningful activities that integrate lesson concepts (e.g., inteviews, letter writing, simulations, models) with language practice opportunities for reading, writing, listening, and/or speaking Comments:

Building Background

7. Concepts explicitly linked to students' background experiences

8. Links explicitly made between past learning and new concepts

9. Key vocabulary emphasized (e.g., introduced, written, repeated, and highlighted for students to see) Comments:

Comprehensible

10. Speech appropriate for students' proficiency level (e.g., slower rate, enunciation, and simple sentence structure for beginners)

11. Clear explanation of academic tasks clear

12. Variety of techniques used to make content concepts clear (e.g., modeling, visuals, hands-on activities, demonstrations, gestures, body language) Comments:

Strategies

13. Ample opportunities for students to use learning strategies

14. Scaffolding techniques consistently used, assisting and supporting student understanding (e.g., think-alouds)

\begin{tabular}{|c|c|c|c|c|c|}
\hline $\begin{array}{l}\text { Highly } \\
\text { Evident }\end{array}$ & & $\begin{array}{c}\text { Somewhat } \\
\text { Evident }\end{array}$ & & $\begin{array}{c}\text { Not } \\
\text { Evident }\end{array}$ & NA \\
\hline 4 & 3 & 2 & 1 & 0 & \\
\hline$\square$ & $\square$ & $\square$ & $\square$ & $\square$ & $\square$ \\
\hline$\square$ & $\square$ & $\square$ & $\square$ & $\square$ & $\square$ \\
\hline$\square$ & $\square$ & $\square$ & $\square$ & $\square$ & $\square$ \\
\hline$\square$ & $\square$ & $\square$ & $\square$ & $\square$ & $\square$ \\
\hline$\square$ & $\square$ & $\square$ & $\square$ & $\square$ & $\square$ \\
\hline$\square$ & $\square$ & $\square$ & $\square$ & $\square$ & $\square$ \\
\hline$\square$ & $\square$ & $\square$ & $\square$ & $\square$ & $\square$ \\
\hline$\square$ & $\square$ & $\square$ & $\square$ & $\square$ & $\square$ \\
\hline$\square$ & $\square$ & $\square$ & $\square$ & $\square$ & $\square$ \\
\hline$\square$ & $\square$ & $\square$ & $\square$ & $\square$ & $\square$ \\
\hline$\square$ & $\square$ & $\square$ & $\square$. & $\square$ & $\square$ \\
\hline$\square$ & $\square$ & $\square$ & $\square$ & $\square$ & $\square$ \\
\hline$\square$ & $\square$ & $\square$ & $\square$ & $\square$ & $\square$ \\
\hline$\square$ & $\square$ & $\square$ & $\square$ & $\square$ & $\square$ \\
\hline
\end{tabular}


Strategies (continued)

15. A variety of questions or tasks that promote higherorder thinking skills (e.g., literal, analytical, and interpretive questions)

Comments:

Interaction

16. Frequent opportunities for interaction and discussion between teacher/student and among students, which encourage elaborated responses about lesson concepts

17. Grouping configurations support language and content objectives of the lesson

18. Sufficient wait time for student response consistently provided

19. Ample opportunities for students to clarify key concepts in $\mathrm{L} 1$ as needed with aide, peer, or $\mathrm{L} 1$ text Comments:

Practice/Application

20. Hands-on materials and/or manipulatives provided for students to practice using new content knowledge

21. Activities provided for students to apply content and language knowledge in the classroom

22. Activities integrate all language skills (i.e., reading, writing, listening, and speaking)

Comments:

Lesson Delivery

23. Content objectives clearly supported by lesson delivery

24. Language objectives clearly supported by lesson delivery

25. Students engaged approximately $90-100 \%$ of the period

26. Pacing of the lesson appropriate to the students' ability level

Comments:

Review/Assessment

27. Comprehensive review of key vocabulary

28. Comprehensive review of key content concepts

29. Regular feedback provided to students on their output (e.g., language, content, work)

30. Student comprehension and learning of all lesson objectives (e.g., spot checking, group response) assessed throughout the lesson

Comments:

\begin{tabular}{cccccc}
$\begin{array}{c}\text { Highly } \\
\text { Evident }\end{array}$ & $\begin{array}{c}\text { Somewhat } \\
\text { Evident }\end{array}$ & $\begin{array}{c}\text { Not } \\
\text { Evident }\end{array}$ & NA \\
\hline 4 & 3 & 2 & 1 & 0 & \\
$\square$ & $\square$ & $\square$ & $\square$ & $\square$ & $\square$
\end{tabular}
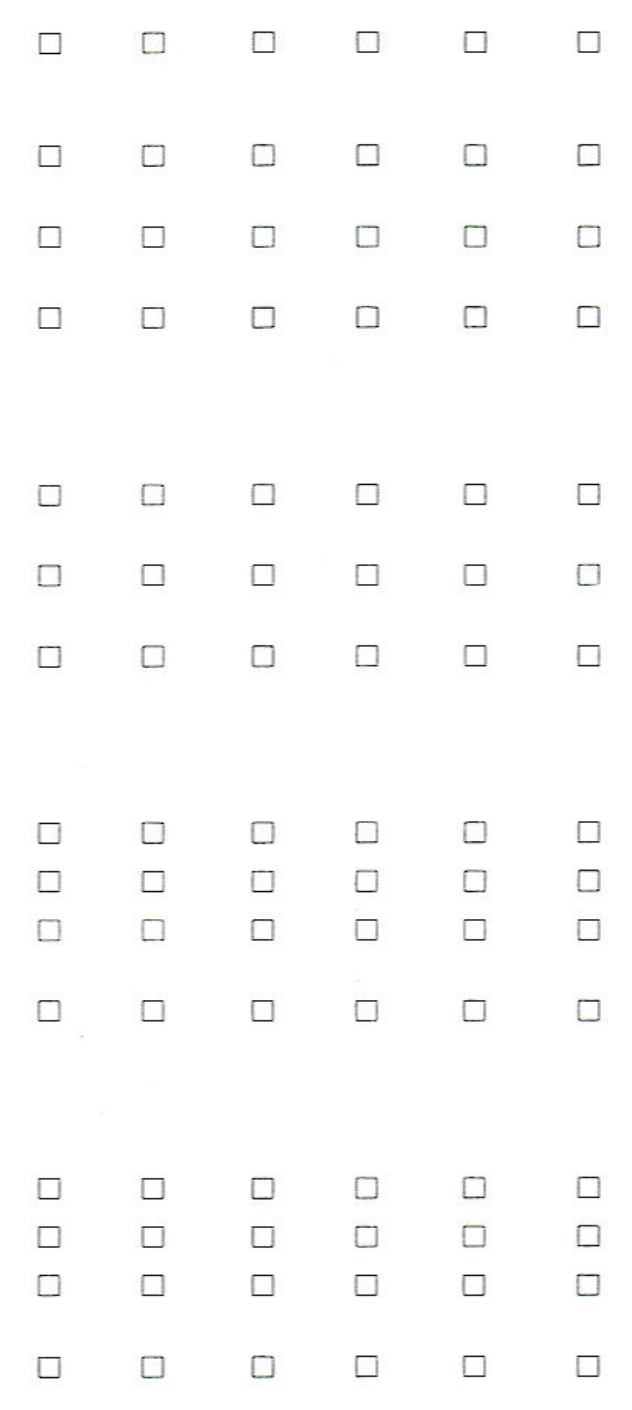

Source: From Jana Echevarria, Maryellen Vogt, \& Deborah Short, Making content comprehensible for English language learners: The SIOP model. Published by Allyn and Bacon, Boston, MA. Copyright (C) 2000 by Pearson Education Inc. Reprinted with permission of the publisher. 


\section{APPENDIX E}

\section{Permission to Use Sheltered Instruction Observation Protocol}

\section{A DODICI}

$\begin{array}{ll}\text { From: } & \text { "A DODICI" } \\ \text { To: } & \text { "MaryEllen Vogt" } \\ \text { Sent: } & \text { Sunday, August 10, } 2008 \text { 2:21 PM } \\ \text { Subject: } & \text { Re: Use of SIOP in Research Stud }\end{array}$

Dear Dr. Vogt

Thank you for granting your permission to use the SIOP in my research study. I will use the SIOP exactly as it is written and published to observe about 30 teachers who have been trained to use sheltered instructional techniques. I'll also make sure to share my results with all three of you. Thank you again for your help.

\section{Adria Dodici}

\section{- Original Message -}

From: MaryEllen Vogt

To: 'Jana Echevarria'; 'A DODICl'

Sent: Friday, August 08, 2008 2:29 PM

Subject: RE: Use of SIOP in Research Study

Hi Adria,

I agree with Jana--we'll look forward to seeing the results of your study! My only request is that you use the SIOP protocol exactly as written (and published), and that you not "adapt" it in any way. Best wishes! mev

MaryEllen Vogt

From: Jana Echevarria

Sent: Wednesday, Auqust 06, 2008 1:12 PM

To: A DODICI.

Subject: Re: Use of SIOP in Research Study

Dear Adria,

Speaking for myself, I welcome your use of the SIOP Model in your research. I have copied Dr. Vogt on this reply. Please keep us posted as to the results.

Best wishes for a speedy process!

Jana Echevarria

A DODICI wrote:

Hello,

My name is Adria Dodici. I am a doctoral student at Portland State University in Portland, OR and am beginning the process of writing my dissertation proposal. I would like to find out if there is a correlation between teachers' multicultural attitudes and their classroom application of sheltered instructional techniques. In the attached letter, I explain my proposed study and how it became of interest to me. I would also like to request your permission (and that of the other SIOP authors) to use the SIOP in my study.

I was able to locate Dr. Deborah Short at CAL, but could not find any current contact information for Dr. Maryellen Vogt. Do you know how I could get in contact with her?

Thank you for considering this request.

Sincerely,

Adria Dodici 


\section{A DODICI}

$\begin{array}{ll}\text { From: } & \text { "A DODICl" } \\ \text { To: } & \text { "Deborah Short" } \\ \text { Sent: } & \text { Tuesday, August 26, 2008 6:51 PM } \\ \text { Subject: } & \text { Re: Use of SIOP in Research Study }\end{array}$

Hello Dr. Short,

Thank you for granting me permission to use the SIOP. I have been trained to use sheltered techniques as well as how to score lessons using the SIOP. For the study, I plan to have at least one other person score with me, in addition to videotaping each observation. Thank you for the suggestion about videotaping and scoring and then scoring again a month or two later. This will work with my plan to do a pilot study. If you have any other suggestions, please let me know.

Sincerely,

Adria Dodici

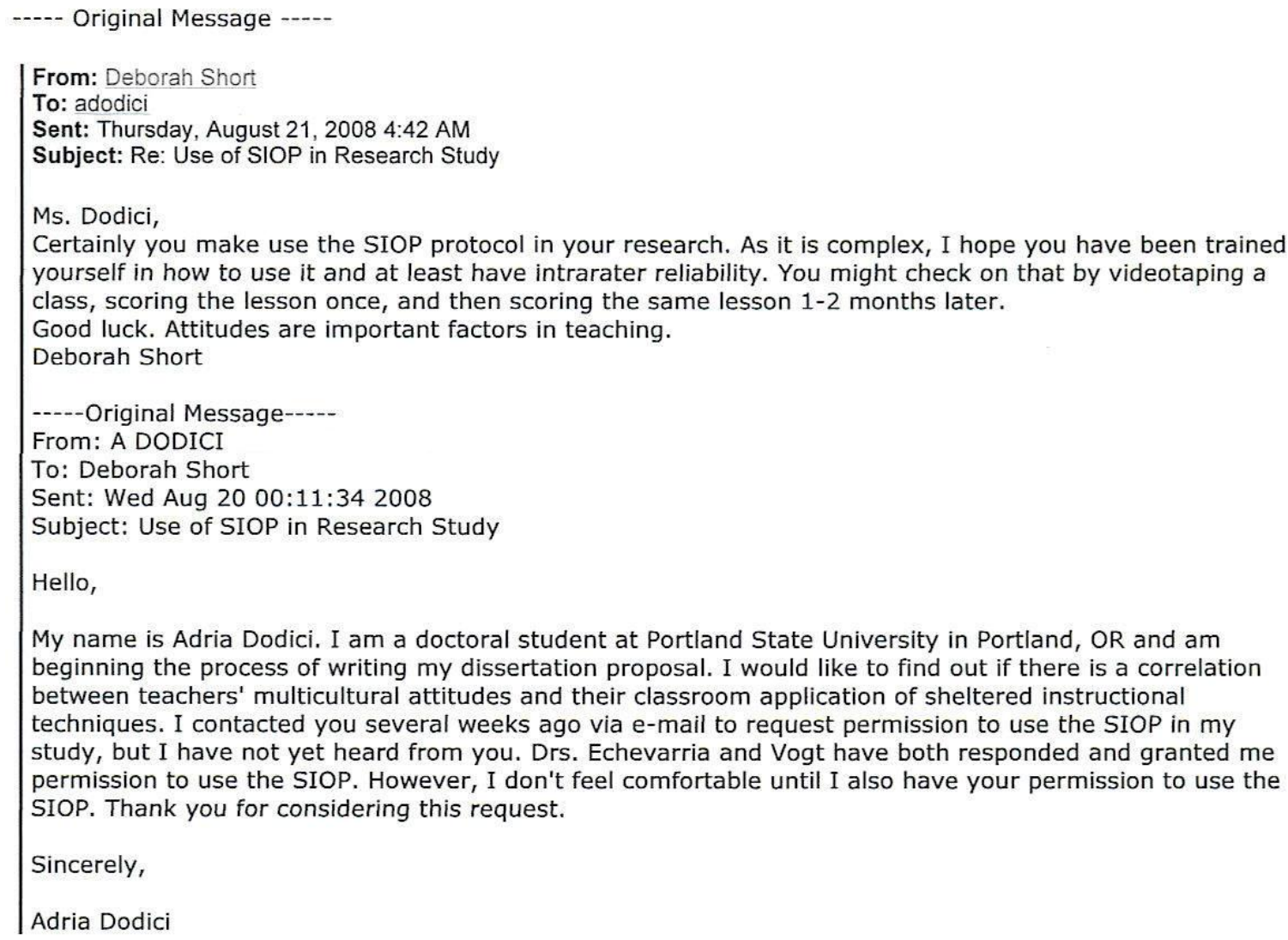




\section{APPENDIX F}

\section{Classroom Instruction Questionnaire}

TO THE PARTICIPANT: Please reflect about and respond to the following questions. Feel free to ask for clarification as needed. You may use the back of this sheet if you need additional space. Because I am merely trying to get an accurate sense of your opinions on these topics, there are no right or wrong answers. Please be assured that this information and all of your responses on this instrument will be kept strictly confidential. Data will be reported so that identification of individuals will be impossible. Your identification number allows this information to be compared with your responses on other measures or observations.

\section{Identification Number:}

Date:

1. What is your opinion on incorporating multicultural education practices (e.g., valuing cultural differences and promoting social justice) into content instruction?

2. What are some of the benefits to incorporating multicultural education practices into content instruction?

3. What are some of the challenges to incorporating multicultural education practices into content instruction? 
4. What do you think are some of the benefits associated with working with students from diverse (e.g., ethnic, linguistic, and socioeconomic) backgrounds?

5. What do you think are some of the challenges associated with working with students from diverse (e.g., ethnic, linguistic, and socioeconomic) backgrounds? 
6. What role do sheltered instructional techniques play in your classroom instruction? Using the table below, place a checkmark in one of the columns to describe how often you utilize each component of sheltered instruction. Then, in the last column please explain why you use the particular sheltered component to the extent you do.

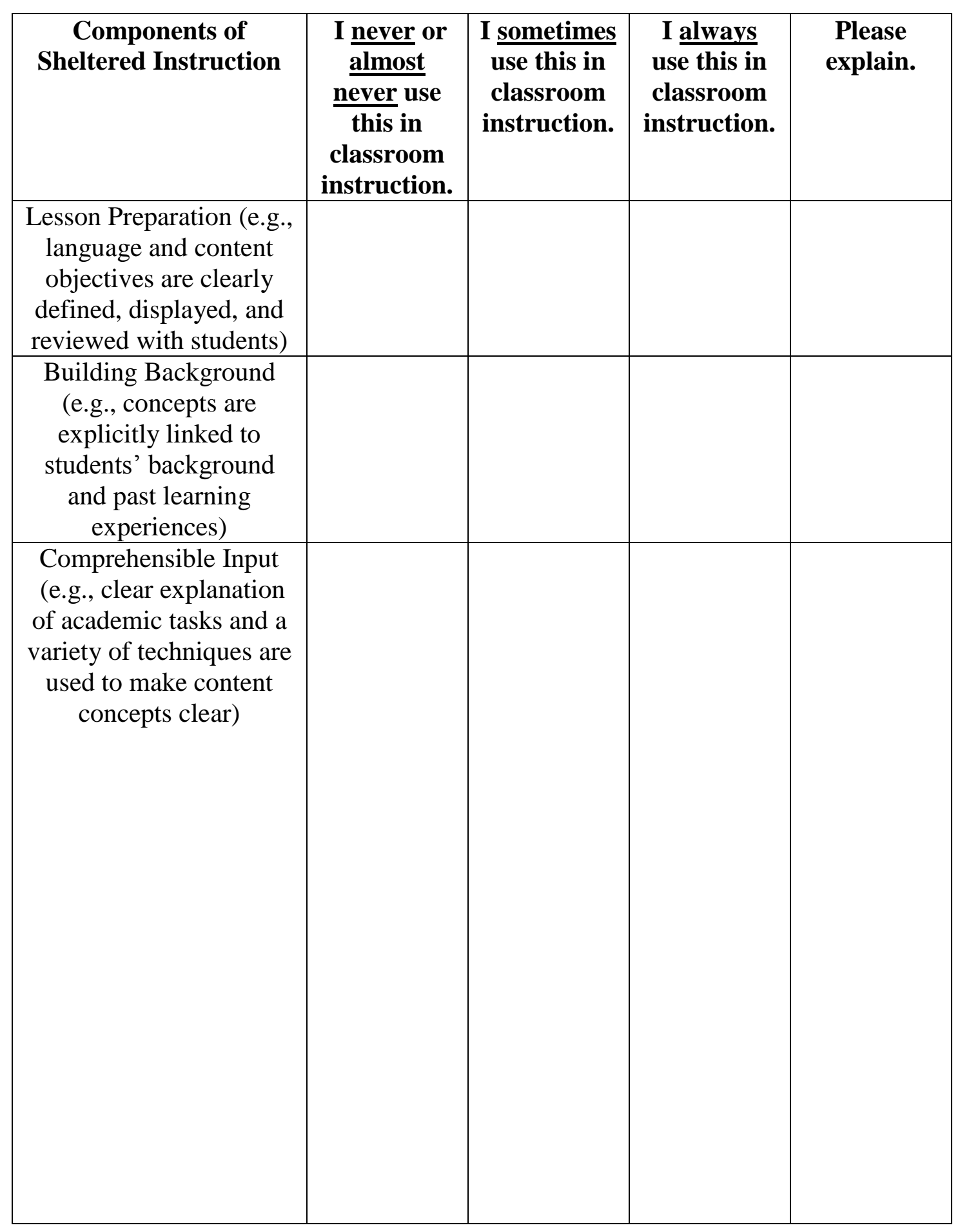




\begin{tabular}{|c|c|c|c|c|}
\hline $\begin{array}{c}\text { Components of } \\
\text { Sheltered Instruction }\end{array}$ & $\begin{array}{l}\text { I never or } \\
\text { almost } \\
\text { never use } \\
\text { this in } \\
\text { classroom } \\
\text { instruction. }\end{array}$ & $\begin{array}{l}\text { I sometimes } \\
\text { use this in } \\
\text { classroom } \\
\text { instruction. }\end{array}$ & $\begin{array}{l}\text { I always } \\
\text { use this in } \\
\text { classroom } \\
\text { instruction. }\end{array}$ & $\begin{array}{l}\text { Please } \\
\text { explain. }\end{array}$ \\
\hline $\begin{array}{c}\text { Strategies } \\
\text { (e.g., students are } \\
\text { frequently provided the } \\
\text { opportunity to use } \\
\text { learning strategies and } \\
\text { scaffolding techniques } \\
\text { are consistently used to } \\
\text { support student } \\
\text { understanding) }\end{array}$ & & & & \\
\hline $\begin{array}{c}\text { Interaction } \\
\text { (e.g., students are } \\
\text { grouped to support } \\
\text { language and content } \\
\text { objectives and have } \\
\text { frequent opportunities } \\
\text { for interaction and } \\
\text { discussion) }\end{array}$ & & & & \\
\hline $\begin{array}{l}\text { Practice/Application } \\
\text { (e.g., activities are } \\
\text { provided for students to } \\
\text { apply content and } \\
\text { language knowledge) }\end{array}$ & & & & \\
\hline $\begin{array}{l}\text { Lesson Delivery } \\
\text { (e.g., content and } \\
\text { language objectives are } \\
\text { clearly supported by } \\
\text { lesson delivery and } \\
\text { pacing of lesson is } \\
\text { appropriate to students' } \\
\text { ability levels) }\end{array}$ & & & & \\
\hline
\end{tabular}




\begin{tabular}{|c|c|c|c|c|}
\hline $\begin{array}{c}\text { Components of } \\
\text { Sheltered Instruction }\end{array}$ & $\begin{array}{c}\text { I never or } \\
\begin{array}{c}\text { almost } \\
\frac{\text { never use }}{\text { this in }} \\
\text { classroom } \\
\text { instruction. }\end{array}\end{array}$ & $\begin{array}{c}\text { I sometimes } \\
\text { use this in } \\
\text { classroom } \\
\text { instruction. }\end{array}$ & $\begin{array}{c}\text { I always } \\
\text { use this in } \\
\text { classroom } \\
\text { instruction. }\end{array}$ & $\begin{array}{c}\text { Please } \\
\text { explain. }\end{array}$ \\
\hline $\begin{array}{c}\text { Review/Assessment } \\
\text { (e.g., comprehensive } \\
\text { review of key } \\
\text { vocabulary and regular } \\
\text { feedback is provided to } \\
\text { students) }\end{array}$ & & & & \\
\hline
\end{tabular}




\section{APPENDIX G}

\section{Semi-structured Interview Questions}

TO THE PARTICIPANT: Thank you for taking the time to share your views about your work and the students you teach. Because I am merely trying to get an accurate sense of your opinions on these topics, there are no right or wrong answers. If at times, you are not sure you understand the question, please ask for clarification. Be assured that your responses on this instrument will be kept strictly confidential. Data will be reported so that identification of individuals will be impossible.

1. What do you think about integrating multicultural education practices (e.g., valuing cultural differences and promoting social justice) into your content area instruction?

2. Tell me about some of your experiences educating students from diverse (e.g., ethnic, linguistic, and socioeconomic) backgrounds?

3. What role does sheltered instruction play in your classroom instruction? Please explain. 


\section{APPENDIX H}

\section{Codebook of Quantitative Data}

Demographic Questionnaire-Single Item Scores

\begin{tabular}{|c|c|c|c|c|}
\hline $\begin{array}{l}\text { Variable } \\
\text { Number }\end{array}$ & Variable & $\begin{array}{l}\text { Type of } \\
\text { Variable }\end{array}$ & Type of Scale & Scoring \\
\hline 1 & Gender & Independent & $\begin{array}{l}\text { Categorical/ } \\
\text { Nominal }\end{array}$ & $\begin{array}{l}1=\text { male } \\
2=\text { female }\end{array}$ \\
\hline 2 & Age & Independent & $\begin{array}{l}\text { Categorical/ } \\
\text { Interval }\end{array}$ & $\begin{array}{l}1=21-40 \\
2=41-60\end{array}$ \\
\hline 3 & Birthplace & Independent & $\begin{array}{l}\text { Categorical/ } \\
\text { Nominal }\end{array}$ & $\begin{array}{l}1=\text { in the U.S. } \\
2=\text { outside the U.S. }\end{array}$ \\
\hline 4 & $\begin{array}{c}\text { Racial/ } \\
\text { Ethnic } \\
\text { Background }\end{array}$ & Independent & $\begin{array}{l}\text { Categorical/ } \\
\text { Nominal }\end{array}$ & $\begin{array}{l}\text { 1=Caucasian race, } \\
\text { American/European ethnicity } \\
\text { 2=Non-Caucasian race, non- } \\
\text { European ethnicity }\end{array}$ \\
\hline 5 & $\begin{array}{l}\text { Religious } \\
\text { Background }\end{array}$ & Independent & $\begin{array}{l}\text { Categorical/ } \\
\text { Nominal }\end{array}$ & $\begin{array}{l}1=\text { Christian } \\
2=\text { none }\end{array}$ \\
\hline 6 & $\begin{array}{c}\text { Physical } \\
\text { Disabilities }\end{array}$ & Independent & $\begin{array}{c}\text { Categorical/ } \\
\text { Nominal }\end{array}$ & $\begin{array}{l}1=\text { none } \\
2=\text { yes }\end{array}$ \\
\hline 7 & $\begin{array}{l}\text { Father's } \\
\text { Education }\end{array}$ & Independent & $\begin{array}{l}\text { Categorical/ } \\
\text { Ordinal }\end{array}$ & $\begin{array}{l}1=\text { completed doctorate } \\
2=\text { completed MS } \\
3=\text { completed BA } \\
\text { 4=completed Assoc./Tech. } \\
5=\text { completed HS/GED } \\
6=\text { did not complete HS }\end{array}$ \\
\hline 8 & $\begin{array}{l}\text { Mother's } \\
\text { Education }\end{array}$ & Independent & $\begin{array}{l}\text { Categorical/ } \\
\text { Ordinal }\end{array}$ & $\begin{array}{l}1=\text { completed doctorate } \\
2=\text { completed MS } \\
3=\text { completed BA } \\
\text { 4=completed Assoc./Tech. } \\
5=\text { completed HS/GED } \\
6=\text { did not complete HS }\end{array}$ \\
\hline 9 & $\begin{array}{c}\text { Socio- } \\
\text { economic } \\
\text { Status (Child) }\end{array}$ & Independent & $\begin{array}{l}\text { Continuous/ } \\
\text { Ratio }\end{array}$ & $\begin{array}{l}1=\text { upper } \\
2=\text { upper middle } \\
3=\text { middle } \\
4=\text { lower middle } \\
5=\text { lower }\end{array}$ \\
\hline 10 & $\begin{array}{l}\text { Socioeconom } \\
\text { ic Status } \\
\text { (Adult) }\end{array}$ & Independent & $\begin{array}{l}\text { Continuous/ } \\
\text { Ratio }\end{array}$ & $\begin{array}{l}1=\text { upper } \\
2=\text { upper middle } \\
3=\text { middle } \\
4=\text { lower middle } \\
5=\text { lower }\end{array}$ \\
\hline
\end{tabular}


Demographic Questionnaire-Single Item Scores (continued)

\begin{tabular}{|c|c|c|c|c|}
\hline $\begin{array}{l}\text { Variable } \\
\text { Number }\end{array}$ & Variable & $\begin{array}{l}\text { Type of } \\
\text { Variable }\end{array}$ & Type of Scale & Scoring \\
\hline 11 & $\begin{array}{l}\text { Completed } \\
\text { Degree }\end{array}$ & Independent & $\begin{array}{c}\text { Categorical/ } \\
\text { Ordinal }\end{array}$ & $\begin{array}{l}1=\text { Bachelor's } \\
2=\text { Master's } \\
3=\text { Doctoral }\end{array}$ \\
\hline 12 & $\begin{array}{l}\text { Specialization } \\
\text { /Major }\end{array}$ & Independent & $\begin{array}{l}\text { Categorical/ } \\
\text { Nominal }\end{array}$ & $\begin{array}{l}1=\text { non-ELL related } \\
\text { 2=ELL related (particular } \\
\text { language, linguistics, bilingual } \\
\text { education, etc.) }\end{array}$ \\
\hline 13 & Endorsement & Independent & $\begin{array}{c}\text { Categorical/ } \\
\text { Nominal }\end{array}$ & $\begin{array}{l}\text { 1=non-ELL related } \\
\text { 2=ELL related (BIL, ESOL, } \\
\text { FOR LANG, GLAD, SIOP, } \\
\text { etc.) }\end{array}$ \\
\hline 14 & $\begin{array}{c}\text { Sheltered } \\
\text { Training }\end{array}$ & Independent & $\begin{array}{c}\text { Continuous/ } \\
\text { Ratio }\end{array}$ & $\begin{array}{l}1=0-54 \\
2=55-109 \\
3=110-164 \\
4=165-219\end{array}$ \\
\hline 15 & $\begin{array}{c}\text { Personal } \\
\text { Study Related } \\
\text { to Sheltered } \\
\text { Instruction }\end{array}$ & Independent & $\begin{array}{c}\text { Categorical/ } \\
\text { Nominal }\end{array}$ & $\begin{array}{l}1=\text { none } \\
2=\text { yes }\end{array}$ \\
\hline 16 & $\begin{array}{l}\text { Number of } \\
\text { Years } \\
\text { Teaching } \\
\text { Middle } \\
\text { School }\end{array}$ & Independent & $\begin{array}{c}\text { Continuous/ } \\
\text { Ratio }\end{array}$ & $\begin{array}{l}1=0-10 \\
2=11-20 \\
3=21-30 \\
4=31-40\end{array}$ \\
\hline 17 & $\begin{array}{c}\text { Number of } \\
\text { Years } \\
\text { Teaching } \\
\text { English } \\
\text { language } \\
\text { learners }\end{array}$ & Independent & $\begin{array}{c}\text { Continuous/ } \\
\text { Ratio }\end{array}$ & $\begin{array}{l}1=0-10 \\
2=11-20 \\
3=21-30 \\
4=31-40\end{array}$ \\
\hline 18 & $\begin{array}{c}\text { Linguistic } \\
\text { Status } \\
\text { (number of } \\
\text { languages } \\
\text { spoken at } \\
\text { intermediate- } \\
\text { fluent level) }\end{array}$ & Independent & $\begin{array}{c}\text { Categorical/ } \\
\text { Nominal }\end{array}$ & $\begin{array}{l}1=\text { monolingual } \\
2=\text { bi/multilingual }\end{array}$ \\
\hline 19 & $\begin{array}{c}\text { Travel } \\
\text { Outside the } \\
\text { U.S. }\end{array}$ & Independent & $\begin{array}{c}\text { Categorical/ } \\
\text { Nominal }\end{array}$ & $\begin{array}{l}1=\text { none } \\
2=\text { yes }\end{array}$ \\
\hline
\end{tabular}


Multicultural Efficacy Scale-Summed Scores

\begin{tabular}{|c|c|c|c|c|}
\hline $\begin{array}{l}\text { Variable } \\
\text { Number }\end{array}$ & Variable & $\begin{array}{l}\text { Type of } \\
\text { Variable }\end{array}$ & $\begin{array}{l}\text { Type of } \\
\text { Scale }\end{array}$ & Scoring \\
\hline 1 & $\begin{array}{c}\text { Section A- } \\
\text { Experience } \\
\text { with People } \\
\text { Different } \\
\text { from You }\end{array}$ & Independent & $\begin{array}{c}\text { Continuous/ } \\
\text { Ordinal }\end{array}$ & $\begin{array}{l}1=\text { never } \\
2=\text { rarely } \\
3=\text { occasionally } \\
4=\text { frequently }\end{array}$ \\
\hline 2 & $\begin{array}{l}\text { Section B- } \\
\text { Opinions }\end{array}$ & Independent & $\begin{array}{c}\text { Continuous/ } \\
\text { Ordinal }\end{array}$ & $\begin{array}{l}\text { Numbers } 8-9 \text { and } 11-14 \\
1=\text { disagree strongly, } 2=\text { disagree } \\
\text { somewhat, } 3=\text { agree somewhat, } \\
\text { 4=agree strongly } \\
\text { Number } 8 \\
1=\text { agree strongly } \\
\text { 2=agree somewhat } \\
3=\text { disagree somewhat } \\
\text { 4=disagree strongly }\end{array}$ \\
\hline 3 & $\begin{array}{l}\text { Section C- } \\
\text { Self- } \\
\text { assessment } \\
\text { of Abilities }\end{array}$ & Independent & $\begin{array}{c}\text { Continuous/ } \\
\text { Ordinal }\end{array}$ & $\begin{array}{l}1=\text { I do not believe I could do this } \\
\text { very well. } \\
2=\text { I could probably do this if I had } \\
\text { to, but it would be difficult for me. } \\
3=\text { I believe I could do this } \\
\text { reasonably well, if I had time to } \\
\text { prepare. } \\
4=I \text { am quite confident that this } \\
\text { would be easy for me to do. }\end{array}$ \\
\hline 4 & $\begin{array}{c}\text { Section D- } \\
\text { Strongest } \\
\text { Belief about } \\
\text { Teaching }\end{array}$ & Independent & $\begin{array}{c}\text { Continuous/ } \\
\text { Ordinal }\end{array}$ & $\begin{array}{l}\text { 1=If every individual learned to } \\
\text { accept and work with every other } \\
\text { person, then there would be no } \\
\text { intercultural problems. } \\
2=\text { If all groups could be helped to } \\
\text { contribute to the general good and } \\
\text { not seek special recognition, we } \\
\text { could create a unified America. } \\
3=\text { All cultural groups are entitled } \\
\text { to maintain their own identity. } \\
4=\text { All cultural groups should be } \\
\text { recognized for their strengths and } \\
\text { contributions. } \\
5=\text { Some groups need to be helped } \\
\text { to achieve equal treatment before } \\
\text { we can reach the goals of a } \\
\text { democratic society. }\end{array}$ \\
\hline
\end{tabular}


Sheltered Instruction Observation Protocol

\begin{tabular}{|c|c|c|c|c|}
\hline $\begin{array}{l}\text { Variable } \\
\text { Number }\end{array}$ & Variable & $\begin{array}{l}\text { Type of } \\
\text { Variable }\end{array}$ & Type of Scale & Scoring \\
\hline 1 & Preparation & Dependent & $\begin{array}{c}\text { Continuous/ } \\
\text { Ratio }\end{array}$ & \multirow{8}{*}{$\begin{array}{l}\text { N/A=-4 from total } \\
\text { possible points } \\
0=\text { not evident } \\
1=\text { in between not evident } \\
\text { and somewhat evident } \\
2=\text { somewhat evident } \\
3=\text { in between somewhat } \\
\text { evident and highly } \\
\text { evident } \\
4=\text { highly evident }\end{array}$} \\
\hline 2 & $\begin{array}{c}\text { Building } \\
\text { Background }\end{array}$ & Dependent & $\begin{array}{c}\text { Continuous/ } \\
\text { Ratio }\end{array}$ & \\
\hline 3 & Comprehensible & Dependent & $\begin{array}{c}\text { Continuous/ } \\
\text { Ratio }\end{array}$ & \\
\hline 4 & Strategies & Dependent & $\begin{array}{l}\text { Continuous/ } \\
\text { Ratio }\end{array}$ & \\
\hline 5 & Interaction & Dependent & $\begin{array}{c}\text { Continuous/ } \\
\text { Ratio }\end{array}$ & \\
\hline 6 & $\begin{array}{c}\text { Practice/ } \\
\text { Application }\end{array}$ & Dependent & $\begin{array}{l}\text { Continuous/ } \\
\text { Ratio }\end{array}$ & \\
\hline 7 & Lesson Delivery & Dependent & $\begin{array}{l}\text { Continuous/ } \\
\text { Ratio }\end{array}$ & \\
\hline 8 & $\begin{array}{c}\text { Review/ } \\
\text { Assessment }\end{array}$ & Dependent & $\begin{array}{l}\text { Continuous/ } \\
\text { Ratio }\end{array}$ & \\
\hline
\end{tabular}




\section{APPENDIX I}

Start List of Codes for Analyzing the Classroom Instruction Questionnaire

MULTICULTURALISM

MUL: SOCIAL JUSTICE

MUL: IMPORTANT TO INCORPORATE IN CONTENT

MUL: IMPROVES TOLERANCE

MUL: IMPROVES MORALITY

MUL: IMPROVES TEACHING \& LEARNING

MUL: IMPROVES THINKING (DIVERGENT, CRITICAL, PROBLEM SOLVING, ETC.)

MUL: IMPROVES INTERPERSONAL SKILLS OF T/S

O Cooperation

○ Reduction of conflict

MUL: INTRAPERSONAL

O Increase students' self-esteem

○ Improve morality

O Improve maturity of students

MUL: PREPARE FOR GLOBAL WORLD

MUL: LEARN

○ Teachers

○ Students

MUL: TIME

○ Not enough

- Takes time

MUL: INSUFFICIENT QUALITY RESOURCES

MUL: INSUFFFICIENT TEACHER KNOWLEDGE AND/OR EXPERIENCE

MUL: NOT A PRIORITY FOR TEACHERS

MUL: RESISTANCE

O By teacher

- By community

- Influence of mainstream culture

MUL: INSUFFIENCENT PROFESSIONAL DEVELOPMENT
MUL

MUL-SJ

MUL-IMP

MUL-TOL

MUL-MOR

MUL-T\&L

MUL-THINK

MUL-SINTERP or

MUL-TINTERP

MUL-INTRA

MUL-GLOBAL MUL-TLRN or

MUL-SLRN

MUL-NTIME

MUL-NRSRC

MUL-NKWEXP

MUL-NPRI

MUL-REST

MUL-NPD 
- Awareness of other perspectives

○ Empathy

DS: LEARN

- Teachers learn about selves

DS-TLRN or

- Students learn about selves

- Teachers learn

DS: GET ALONG WITH OTHERS

DS: BETTER UNDERSTANDING OF WORLD

DS: IMPROVE TEACHING

DS: GENERAL BENEFIT (Interpersonal \& Intrapersonal)

DS-SLRN

DS: INCREASE FLEXIBILITY IN RESPONSES

DS: STUDENTS EXPERIENCE INJUSTICE

DS: INSUFFICIENT MATERIALS/AVAILABILITY

DS: TIME

- Insufficient time for preparation

DS-GTALNG

DS-UNDRWRLD

DS-TCHNG

DS-INTER

DS-INTRA

DS-FLEXRSP

DS-INJUST

DS-NMAT

DS-NTME

- Less instructional time

- Not enough time to give students attention they need

DS: CULTURAL MISUNDERSTANDINGS/DIFFERENCES

○ Teacher is stereotyped

- Language (family and teacher aren't proficient in same language)

O Offending students

- Teacher goals don't match that of students or families

DS: TEACHING IS MORE COMPLEX

DS-CMPLXTCH

- Assessing prior knowledge is more difficult

- Teaching language and content

DS: LACK OF KNOWLEDGE/EXPERIENCE

DS: INSUFFICIENT PROFESSIONAL DEVELOPMENT

DS-NKNEX

DS-NPD 


\section{APPENDIX J}

Distributions of the Multicultural Efficacy Scale-Skewness and Kurtosis

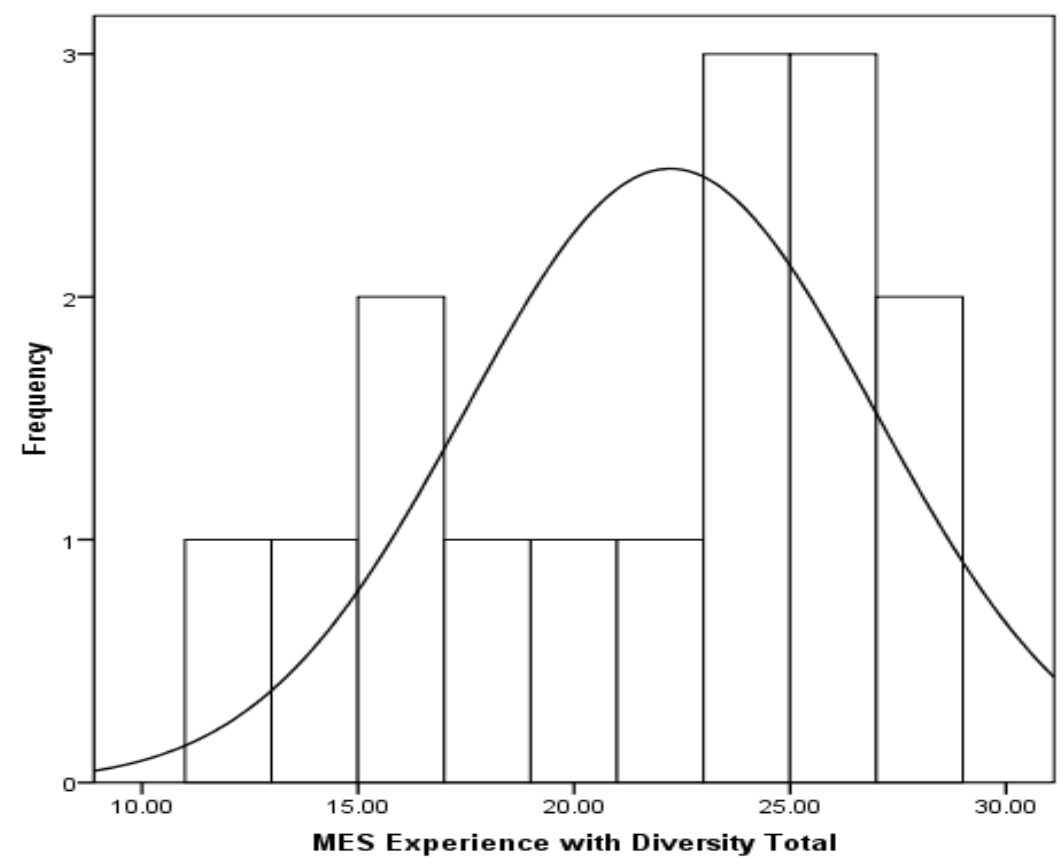

Mean $=21.07$
Std. Dev. $=5.147$
$N=15$

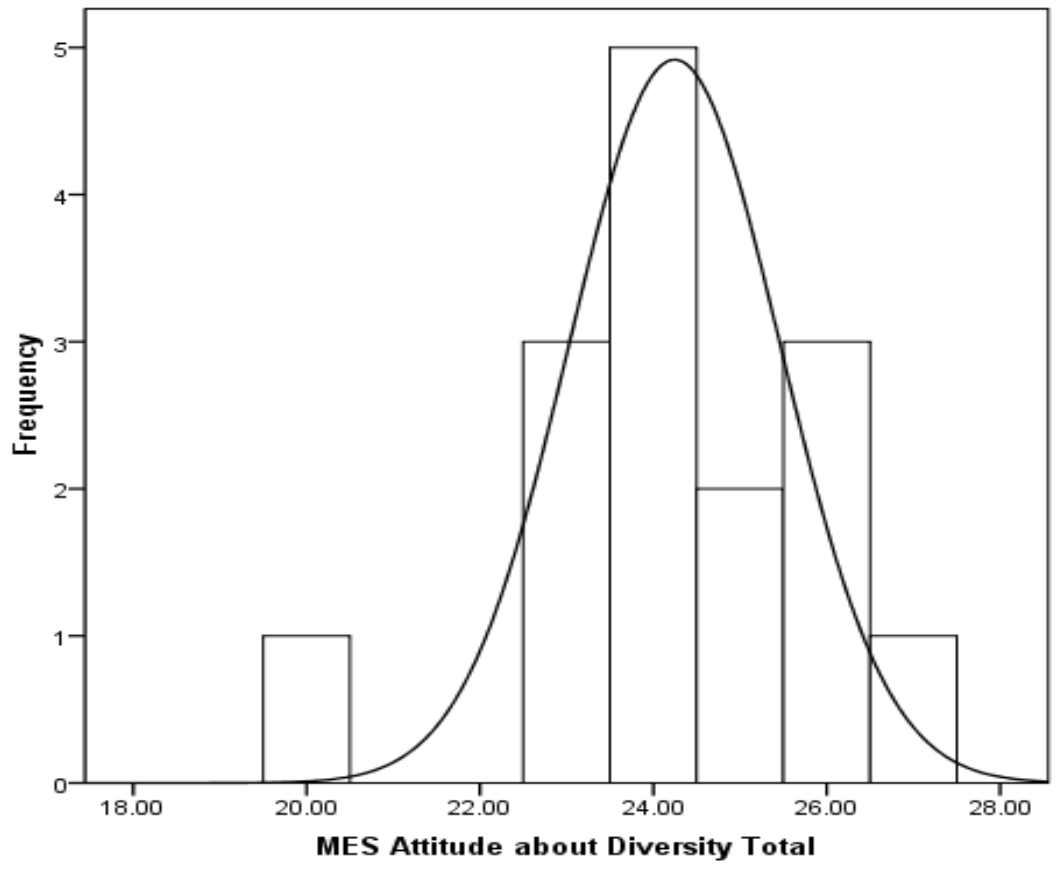

Mean $=24.27$ Std. Dev $=1.71$ $N=15$ 


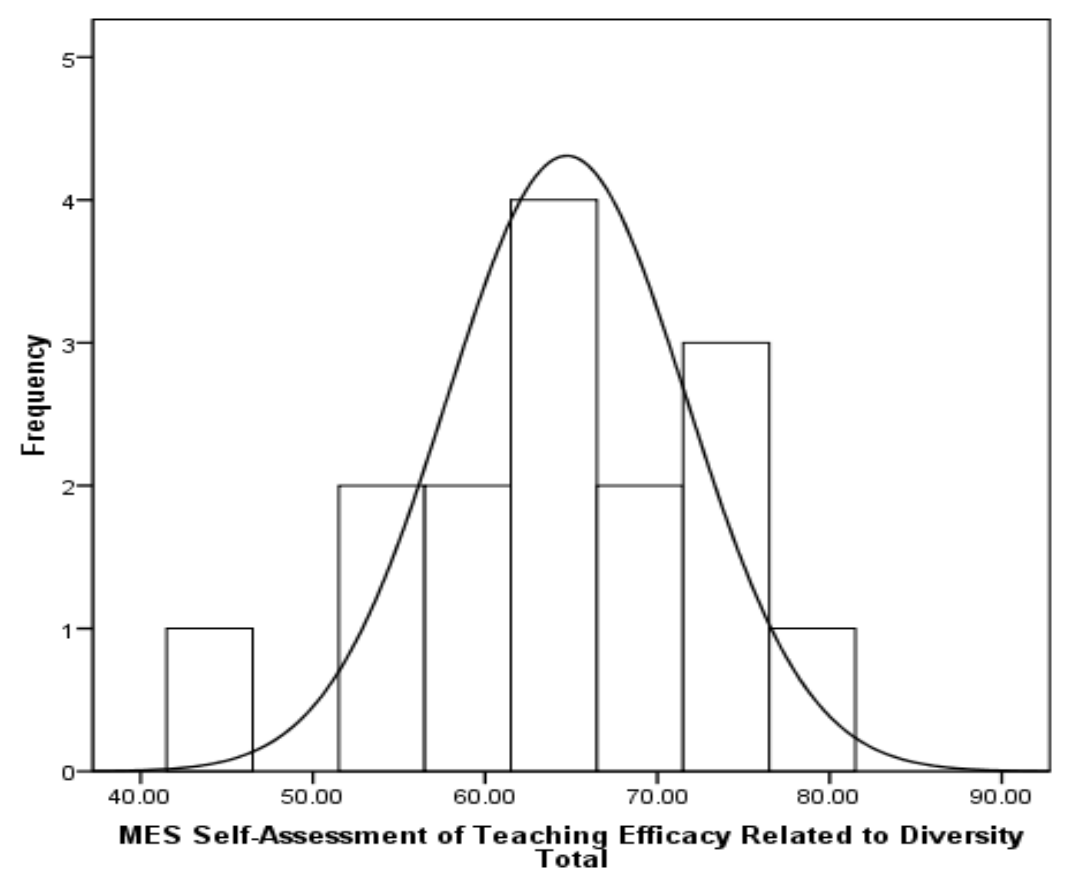

Mean $=64.53$

$\mathrm{N}=15$
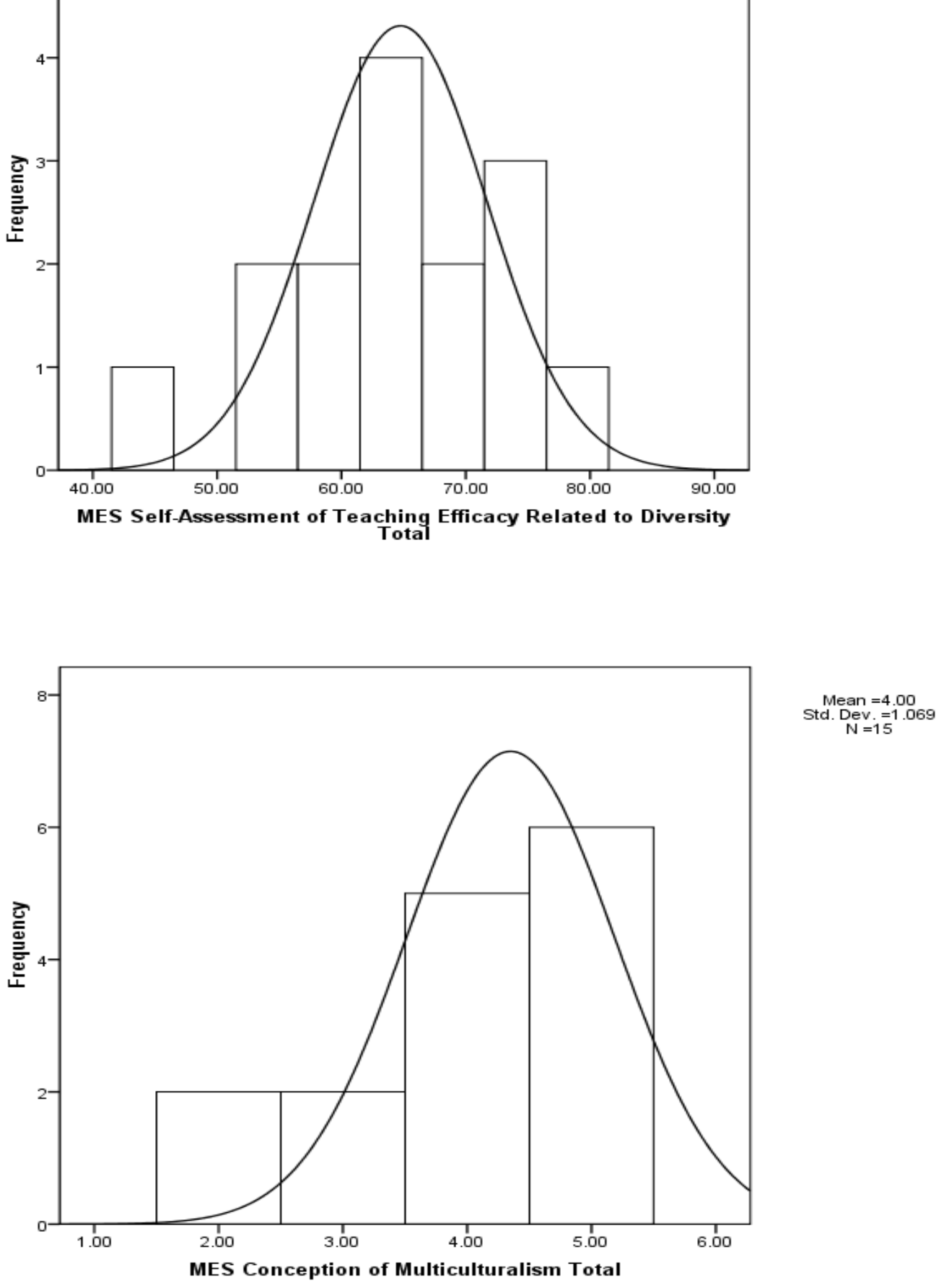